\title{
A Higher-Order Distributed Calculus with Name Creation
}

\author{
Adrien Piérard \\ Tohoku University \\ Email: adrien@kb.ecei.tohoku.ac.jp
}

\author{
Eijiro Sumii \\ Tohoku University \\ Email: sumii@kb.ecei.tohoku.ac.jp
}

\begin{abstract}
This paper introduces $\mathrm{HO} \pi \mathrm{Pn}$, the higher-order $\pi$ calculus with passivation and name creation, and develops an equivalence theory for this calculus. Passivation [Schmitt and Stefani] is a language construct that elegantly models higherorder distributed behaviours like failure, migration, or duplication (e.g. when a running process or virtual machine is copied), and name creation consists in generating a fresh name instead of hiding one. Combined with higher-order distribution, name creation leads to different semantics from name hiding, and is closer to implementations of distributed systems. We define for this new calculus a theory of sound and complete environmental bisimulation to prove reduction-closed barbed equivalence and (a reasonable form of) congruence. We furthermore define environmental simulations to prove behavioural approximation, and use these theories to show non-trivial examples of equivalence or approximation. Those examples could not be proven with previous theories, which were either unsound or incomplete under the presence of process duplication and name restriction, or else required universal quantification over general contexts.
\end{abstract}

\section{INTRODUCTION}

Background: With the increasing call for fault tolerance, on-demand computational power and better responsiveness, higher-order and distribution are pervasive in today's computing environment. In this paper, we call higher-order the ability to send and receive processes through communication channels, and distribution the possibility of location-dependant behaviour. For example, Dell and Hewlett Packard sell products with virtual machine live migration [17], [5], and Gmail relies on remote execution of JavaScript in the users' browsers. Yet, despite the ubiquity and importance of such higher-order distributed systems, the inherent complexity of these systems makes them difficult to analyse, and thus subject to bugs. Therefore, formal models and methods that help reason about higher-order distribution are sought after.

Passivation [18], [6], [8], [11] is a language abstraction for elegantly modelling higher-order distributed systems in process calculi based on the higher-order $\pi$-calculus [12], [15] (with which we assume our reader's familiarity). In its simplest form, passivation consists of a syntax of located processes $l[P]$, where $l$ is a name called a location and $P$ is a process located at $l$, and two labelled transition rules, $l[P] \stackrel{\alpha}{\rightarrow} l\left[P^{\prime}\right]$ if $P \stackrel{\alpha}{\longrightarrow} P^{\prime}$ (TRANSP), and $l[P] \stackrel{\bar{l}\langle P\rangle}{\longrightarrow} 0$ (PASSIV), where the relation $P \stackrel{\alpha}{\rightarrow} Q$ in general reads " $P$ does action $\alpha$ and becomes Q." The TRANSP rule states that locations are transparent, i.e. do not hide any transition $\alpha$ of the processes they are hosting. The PASSIV rule shows how a located process can be passivated, i.e. stopped and output to a channel of the same name as the location.

Despite its simplicity, passivation is yet powerful enough to model complex higher-order distributed behaviours. For example, one can conveniently model failure of a process $P$ at location $l$ as $l[P]|l(X) \cdot \overline{\text { fail }} \rightarrow 0| \overline{\text { fail }}$, migration from location $l$ to location $m$ as $l[P]|l(X) \cdot m[X] \rightarrow 0| m[P]$, or duplication as $l[P]|l(X) .(l[X] \mid l[X]) \rightarrow 0| l[P] \mid l[P]$.

Name creation versus restriction: To our knowledge, previous process calculi with passivation - or, more generally, with higher-order distribution (i.e. communication of processes through channels across locations)-all used so-called name restriction [6], [18], [8], [11]. It hides names, forbidding reactions like $\bar{a} . Q\left|\nu a .\left(a . R_{1} \mid \bar{a} . R_{2}\right) \rightarrow Q\right| \nu a .\left(R_{1} \mid \bar{a} . R_{2}\right)$, where the syntax $\nu a . P$ in general means that name $a$ is local to process $P$, hidden from the outside. Although the name $a$ is textually present in the process $\bar{a} . Q$ above, the $a$ under the $\nu$ operator is only visible to $a . R_{1}$ and $\bar{a} \cdot R_{2}$, hence not usable for synchronisation with $\bar{a} . Q$.

Nonetheless, sharing of hidden names is possible via name extrusion, as in the reaction $l[\nu a . \bar{c}\langle a\rangle . Q] \mid m[c(x) \cdot R] \rightarrow \nu a$. $(l[Q] \mid m[R\{a / x\}])$. This reaction shows that the name $a$, which was local to location $l$, can be sent on another channel $c$ and shared with the receiver outside $l$. In other words, extrusion extends the scope of the sent name to contain the receiver too, possibly crossing location boundaries outwards.

This makes name restriction harder to implement in higherorder distributed settings, as one needs to maintain the scope of extruded names across physically different locations. For example, guaranteeing that the process $\nu a .(l[Q] \mid m[R\{a / x\}])$ above cannot interfere with another process that coincidentally uses the same name $a$ seems to require somehow keeping global information about its scope.

By contrast, real implementations of distributed systems often use name creation [19], which (perhaps against common belief) leads to a different equivalence theory from that of name restriction. Our name creation consists in forbidding transitions under a $\nu$ operator and in generating a fresh name as an internal transition step, shown in the following rule

$$
\frac{a \notin s}{s \vdash \nu a . P \stackrel{\tau}{\rightarrow} s \cup\{a\} \vdash P} \text { CREATE }
$$

where the syntax $s \vdash Q$ in general reads "process $Q$, given 
a set $s$ of already created names." This rule means that the process $\nu a . P$ can, in an internal transition step, create a name $a$ that is stored immediately in the set of created names, and continue as process $P$. Assuming that we identify processes up-to alpha-conversion of names bound by the $\nu$ operator, the clause $a \notin s$ is a simple precaution to guarantee that freshly created names are indeed unique.

The creationist treatment of names makes the semantics closer to implementations: for example, suppose name creation is the generation of random numbers (arguably unique). Then, two different names in the model would actually be different numbers in the implementation too, thus ruling out interferences between processes and eliminating the need for explicit scope information.

Equivalence of higher-order distributed processes with name creation differs from that with name restriction. Concretely, consider the process

$$
P=\nu l .(l[\nu a \cdot(a \mid \bar{a} \cdot \bar{a} \cdot \bar{\omega})] \mid l(X) \cdot(X \mid X))
$$

which, with name restriction semantics, can at best reduce (in several steps) to $\nu l .(0|\nu a .(0 \mid \bar{a} \cdot \bar{\omega})| \nu a .(0 \mid \bar{a} \cdot \bar{\omega}))$. With name creation semantics, there is also a reduction sequence that leads to the exhibition of name $\omega$ by having creation of name $a$ happen before passivation and duplication:

$$
\begin{array}{rlll} 
& \{\omega\} & \vdash \nu l \cdot(l[\nu a \cdot(a \mid \bar{a} \cdot \bar{a} \cdot \bar{\omega})] \mid l(X) \cdot(X \mid X)) \\
\rightarrow & \{\omega, l\} & \vdash l[\nu a \cdot(a \mid \bar{a} \cdot \bar{a} \cdot \bar{\omega})] \mid l(X) \cdot(X \mid X)(\text { create } l) \\
\rightarrow & \{\omega, l, a\} & \vdash l[a \mid \bar{a} \cdot \bar{a} \cdot \bar{\omega}] \mid l(X) \cdot(X \mid X) & \text { (create } a) \\
\rightarrow & \{\omega, l, a\} & \vdash 0|a| \bar{a} \cdot \bar{a} \cdot \bar{\omega}|a| \bar{a} \cdot \bar{a} \cdot \bar{\omega} & \text { (react on } l) \\
\rightarrow & \{\omega, l, a\} & \vdash 0|0| \bar{a} \cdot \bar{\omega}|a| \bar{a} \cdot \bar{a} \cdot \bar{\omega} & \text { (react on } a) \\
\rightarrow & \{\omega, l, a\} & \vdash 0|0| \bar{\omega}|0| \bar{a} \cdot \bar{a} \cdot \bar{\omega} & \text { (react on } a)
\end{array}
$$

Similarly, another (perhaps surprising) difference is the nonbisimilarity between $l[\nu a . \nu b . P]$ and $l[\nu b . \nu a . P]$ with $P=a . b$. $a \cdot \overline{\omega_{1}} \mid \bar{a} \cdot \bar{b} \cdot \bar{b} \cdot \overline{\omega_{2}}$, which are indistinguishable under name restriction. To see it, suppose $s \vdash l[\nu a . \nu b . P]$ with $s=\left\{l, \omega_{1}, \omega_{2}\right\}$ creates name $a$ and is duplicated (i.e. is passivated and then spawned twice), after which the $b$ of each copy is created, giving $s^{\prime} \vdash\left(a \cdot b_{1} \cdot a \cdot \overline{\omega_{1}} \mid \bar{a} \cdot \overline{b_{1}} \cdot \overline{b_{1}} \cdot \overline{\omega_{2}}\right) \mid\left(a \cdot b_{2} \cdot a \cdot \overline{\omega_{1}} \mid \bar{a} \cdot \overline{b_{2}} \cdot \overline{b_{2}} \cdot \overline{\omega_{2}}\right)$ with $s^{\prime}=s \cup\left\{a, b_{1}, b_{2}\right\}$. Then, this process can exhibit $\overline{\omega_{1}}$ using both $\bar{a}$ 's and a $\bar{b}_{1}$, giving $s^{\prime} \vdash\left(\overline{\omega_{1}} \mid \overline{b_{1}} \cdot \overline{\omega_{2}}\right) \mid\left(a \cdot b_{2} \cdot a\right.$. $\left.\overline{\omega_{1}} \mid \overline{b_{2}} \cdot \overline{b_{2}} \cdot \overline{\omega_{2}}\right)$. Yet, it cannot exhibit $\overline{\omega_{2}}$ which is guarded by two $\overline{b_{2}}$ 's while there is only one $b_{2}$. In order for $l[\nu b . \nu a$. $P]$ to weakly follow and exhibit $\overline{\omega_{1}}$ too, it must also share $a$, i.e. create it before duplication, forcing the creation and sharing of $b$ as well. This gives $s \cup\{a, b\} \vdash\left(a . b \cdot a \cdot \bar{\omega}_{1} \mid \bar{a} \cdot \bar{b} \cdot \bar{b}\right.$. $\left.\overline{\omega_{2}}\right) \mid\left(a \cdot b \cdot a \cdot \overline{\omega_{1}} \mid \bar{a} \cdot \bar{b} \cdot \bar{b} \cdot \overline{\omega_{2}}\right)$ which weakly exhibits not only $\overline{\omega_{1}}$ but also $\overline{\omega_{2}}$, therefore telling apart $l[\nu a . \nu b . P]$ and $l[\nu b . \nu a . P]$. More non-bisimilarities because of name creation semantics are discussed in Section VI. (We will note, however, that these processes are still mutually similar.)

In this paper, we argue that name creation is a realistic alternative to name restriction when modelling higher-order distribution. We recall that a restriction-based semantics is harder to implement, because of the difficulty of implementing distributed scope (which is inherent to the communication of bound names). Here, we discuss several of such semantics and their additional differences from name creation in a higherorder distributed setting. (i) A structural congruence rule $a[\nu c$. $P] \equiv \nu c . a[P]$ (cf. [1]). Under the presence of process duplication, it is "unsound," i.e. makes some inequivalent processes structurally congruent. For example, it allows $a[\nu c .(\bar{c} \mid c . c$. $\bar{\omega})] \equiv \nu c \cdot a[\bar{c} \mid c \cdot c \cdot \bar{\omega}]$, but the two processes are distinguished by an observer $R=a(X) .(X \mid X)$. (ii) Enforcing extrusion [18] before passivation like $l[\nu c . P] \mid l(X) .(m[X] \mid n[X]) \rightarrow \nu c$. $(m[P] \mid n[P])$. It does not allow duplication without sharing private channels, keeping passivation from being used as a general device for copying. (iii) Forbidding passivation when $\nu$ is in evaluation position, i.e. $l[\nu c . P] \stackrel{\bar{l}\langle\nu c . P\rangle}{\leftrightarrow} 0$. It hinders duplication with private channels as well. Moreover, expectedly equivalent processes $l[\nu a . a[P]]$ and $l[P]$ are distinguished by $l(X) . \bar{\omega}$, which reacts only with $l[P]$. (iv) Vertical extrusion with an extra rule like $l[\nu c . P] \stackrel{\tau}{\rightarrow} \nu c . l[P]$. It differs from name creation too: consider $Q=l[m[\nu a . P] \mid m(X) .(X \mid X)] \mid l(Y)$. $(Y \mid Y)$ which can become $Q^{\prime}=l[\nu a . m[P] \mid m(X) .(X \mid X)] \mid$ $l(Y) .(Y \mid Y)$ in a step, and then (weakly) become either $\nu a$. $(P|P| P \mid P)$ with one bound name, or $\nu a .(P \mid P) \mid \nu a .(P \mid P)$ with two. With name creation, there is no reduction $Q \Rightarrow Q^{\prime}$ such that only $Q^{\prime} \Rightarrow P\left\{a_{1} / a\right\}\left|P\left\{a_{1} / a\right\}\right| P\left\{a_{1} / a\right\} \mid P\left\{a_{1} / a\right\}$ and $Q^{\prime} \Rightarrow\left(P\left\{a_{1} / a\right\} \mid P\left\{a_{1} / a\right\}\right) \mid\left(P\left\{a_{2} / a\right\} \mid P\left\{a_{2} / a\right\}\right)$ with $a_{1}$ and $a_{2}$ fresh, whence the difference.

Equivalence and inequivalence in higher-order distribution: We have just seen that equivalence differs depending on the semantics of names. Consequently, the equivalence theory under the presence of name creation needs to be rethought. Behavioural equivalence can be characterised as reduction-closed barbed equivalence (or congruence) [7] which has a simple definition but is impractical as a proof method because of a universal quantification on observer processes (or contexts) in its definition. Therefore, more convenient relations like bisimulations, whose membership implies reduction-closed barbed equivalence and which come with a co-inductive proof method, are sought after.

Accordingly, we define a theory of (environmental [20], [21], [14], [16], [11]) bisimulation for a higher-order $\pi$ calculus with both passivation and name creation. The theory is proven to be sound and, thanks to name creation semantics, complete. (In contrast, environmental bisimulations for higher-order $\pi$-calculus with passivation [11] were far from complete under name restriction semantics, being unsound without severe constraints on environments.) It can then be used to prove non-trivial equivalences that could not be shown previously [11], [8], like that of distributed left and right list folds (simplified versions of "MapReduce" [3]), detailed in Section V.

One may also want to prove bisimilarity of distributed programs that are more structurally different than the two fold functions, e.g. linear and logarithmic implementations of power functions. Perhaps surprisingly again, these implementations are not bisimilar. The reason is that the linear distributed implementation uses more hosts than the logarithmic one, and is therefore more likely to fail (under either of name 
creation and restriction). Thus, bisimilarity may sometimes be too strong an equivalence. Instead, mutual simulation can be desirable, so as to provide a coarser equivalence (cf. [9, p. 20, Exercise 3.10]) still useful for comparing such programs. Environmental simulations can be proven, for example, between distributed linear and logarithmic power functions as detailed in Section VI-B.

Summary of our contributions: In this paper, we introduce the higher-order $\pi$-calculus with passivation and name creation (henceforth $\mathrm{HO} \pi \mathrm{Pn}$ ) a dialect of $\mathrm{HO} \pi \mathrm{P}$ (the higherorder $\pi$-calculus with passivation and name restriction) [8]. We then provide environmental bisimulations that are sound and complete with respect to reduction-closed barbed equivalence and (a reasonable form of) congruence, and use them to prove a non-trivial equivalence that could not be shown with previous methods. We also provide sound environmental simulations that can be used to show reduction-closed barbed approximation, and give a non-trivial simulation proof as well.

Overview of the paper: The rest of the paper is structured as follows. Section II defines $\mathrm{HO} \pi \mathrm{Pn}$. Section III formalises our environmental bisimulations and simulations, and Section IV establishes their soundness and completeness. Section $\mathrm{V}$ shows the example bisimilarity proof of distributed left and right folds. Section VI discusses non-bisimilarity in higher-order distribution and shows the simulation proof of distributed power functions. Finally, Section VII considers previous and future work.

\section{Higher-ORder $\pi$-CAlCUlus With Passivation AND NAME CREATION}

We formally introduce $\mathrm{HO} \pi \mathrm{Pn}$ through its syntax and labelled transition system. The syntax of $\mathrm{HO} \pi \mathrm{Pn}$ processes $P, Q$ and terms $M, N$ is given by the following grammar (the same as in [11]):

$$
\begin{aligned}
P, Q \quad::= & 0|a(X) . P| \bar{a}\langle M\rangle . P|(P \mid Q)| a[P] \\
& |=\nu a . P| ! P \mid \operatorname{run}(M) \\
M, N \quad:= & X \mid ' P
\end{aligned}
$$

Briefly, $X$ ranges over the set of variables, and $a$ over names. 0 is a stuck process, $a(X)$ and $\bar{a}\langle M\rangle$ input and output prefixes, | the parallel composition operator, and $a[P]$ the process $P$ located at location $a . \nu a$ is the name creation prefix, ! the replication operator, run the thawing operator which is used to turn a term into a process, $X$ a variable and ' $P$ a process as a term. As in [11], the distinction between processes and terms is needed for our generic up-to context technique (see Section III).

The semantics of $\mathrm{HO} \pi \mathrm{Pn}$ is given by the following labelled transitions system which is based on that of the higher-order $\pi$-calculus with passivation [8] -itself being based on that of the higher-order $\pi$-calculus [12] — and is now defined on configurations. A configuration $s \vdash P$ is the pair of a set $s$ of names and a process $P$ such that $f n(P) \subseteq s$. We casually write $s x$ or $s, x$ for $s \cup\{x\}$ or $s \cup x$ when $x$ is a name or a set of names. Omitting symmetric rules PAR-R and REACT$R$, the transition relation is defined inductively by the rules in

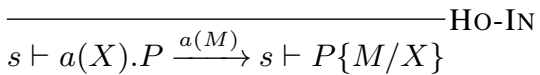

$$
\begin{aligned}
& s \vdash \bar{a}\langle M\rangle . P \stackrel{\bar{a}\langle M\rangle}{\longrightarrow} s \vdash P^{\text {Ho-OUT }} \\
& \frac{s \vdash P \stackrel{\alpha}{\rightarrow} s^{\prime} \vdash P^{\prime} \quad\left(s^{\prime} \backslash s\right) \cap f n(Q)=\emptyset}{s \vdash P\left|Q \stackrel{\alpha}{\rightarrow} s^{\prime} \vdash P^{\prime}\right| Q} \text { PAR-L } \\
& \frac{s \vdash P \stackrel{\bar{a}\langle M\rangle}{\rightarrow} s \vdash P^{\prime} \quad s \vdash Q \stackrel{a(M)}{\longrightarrow} s \vdash Q^{\prime}}{s \vdash P\left|Q \stackrel{\tau}{\rightarrow} s \vdash P^{\prime}\right| Q^{\prime}} \text { REACT-L } \\
& \frac{s \vdash ! P \mid P \stackrel{\alpha}{\rightarrow} s^{\prime} \vdash P^{\prime}}{s \vdash ! P \stackrel{\alpha}{\rightarrow} s^{\prime} \vdash P^{\prime}} \text { REP } \frac{a \notin s}{s \vdash \nu a . P \stackrel{\tau}{\rightarrow} s, a \vdash P} \text { CREATE } \\
& \frac{s \vdash P \stackrel{\alpha}{\rightarrow} s^{\prime} \vdash P}{s \vdash a[P] \stackrel{\alpha}{\rightarrow} s^{\prime} \vdash a\left[P^{\prime}\right]} \text { TRANSP } \\
& \longrightarrow \text { PASSIV } \stackrel{s \vdash \operatorname{run}\left({ }^{\prime} P\right) \stackrel{\tau}{\rightarrow} s \vdash P}{\longrightarrow} \text { RUN }
\end{aligned}
$$

Fig. 1. Labelled transitions system of $\mathrm{HO} \pi \mathrm{Pn}$

Figure 1. Assuming knowledge of the standard higher-order $\pi$ calculus [15], [12], [13], we skim over the distribution-related transitions and comment on the notable changes coming from name creation. The TRANSP rule expresses the transparency of locations - the fact that transitions can happen inside a location and be observed outside its boundaries. The PASSIV rule shows how a process running inside a location can be passivated, i.e. stopped, turned into a term, and sent along a channel whose name corresponds to that of the location. The RUN rule shows how to retrieve a process from a term at the cost of an internal transition.

The rule CREATE shows how a process $\nu a . P$ can create a name $a$-which is added to the configuration's set of namesand become $P$ in an internal transition step. As we identify processes up-to alpha-conversion of bound names, progress is guaranteed. The rule PAR-L shows that a transition can happen in a sub-process provided it does not create a name that is free in another sub-process put in parallel (the function $f n$, which returns the set of free names of a process or a term, is standard). Again, alpha-conversion is used for guaranteeing that no free name of $Q$ will be captured.

The other rules are straightforward even in the presence of name creation and will not be discussed further. As usual with small-step semantics, when the assumptions cannot be satisfied or when a case is undefined (as in $\operatorname{run}(X)$ ), transition does not progress and the process is stuck.

Henceforth, we shall write $\bar{a} . P$ for $\bar{a}\langle 0\rangle . P$ and $a . P$ for $a(X) . P$ when $X$ is not free in $P$. We define structural congruence $\equiv$ as the smallest congruence on processes with $P \equiv P\left|0, P_{1}\right|\left(P_{2} \mid P_{3}\right) \equiv\left(P_{1} \mid P_{2}\right)\left|P_{3}, P_{1}\right| P_{2} \equiv P_{2} \mid P_{1}$ and $! P \equiv ! P \mid P$. Notice that this definition is not standard: it allows neither $(\nu a . P) \mid Q \equiv \nu a .(P \mid Q), \nu a . \nu b . P \equiv \nu b . \nu a . P$, nor $\nu a .0 \equiv 0$. 


\section{ENVIRONMENTAL Bisimulation AND SimUlation $\mathrm{FOR} \mathrm{HO} \pi \mathrm{PN}$}

We define an environmental relation $\mathcal{X}$ as a set of sextuples $(\mathcal{E}, r, s, P, t, Q)$ where $\mathcal{E}$ is a binary relation (called the environment) on terms with no free variables and finitely many free names, $r$ is a finite set of names (the public names), $s$ and $t$ too are finite sets of names (the created names) such that $r \subseteq s \cap t$, and $P$ and $Q$ are variable-closed processes (the tested processes). We often write $(s \vdash P) \mathcal{X}_{\mathcal{E} \cdot r}(t \vdash Q)$ to mean $(\mathcal{E}, r, s, P, t, Q) \in \mathcal{X}$ for an environmental relation $\mathcal{X}$.

Definition 1. We define multi-hole contexts for terms $C$ (contexts that have holes for terms) and multi-hole contexts for processes $C_{p}$ (contexts that have holes for processes) as:

$$
\begin{aligned}
& C::=0|a(X) \cdot C| \bar{a}\langle D\rangle \cdot C|(C \mid C)| a[C]|\nu a . C| ! C \mid \operatorname{run}(D) \\
& D::=[\cdot]_{i}|X|{ }^{C} C \\
& C_{p}:=[\cdot]_{i}|0| a(X) \cdot C_{p}\left|\bar{a}\left\langle D_{p}\right\rangle \cdot C_{p}\right|\left(C_{p} \mid C_{p}\right)\left|a\left[C_{p}\right]\right| \nu a . C_{p} \\
&\left|! C_{p}\right| \operatorname{run}\left(D_{p}\right) \\
& D_{p}::=X \mid{ }^{\prime} C_{p}
\end{aligned}
$$

Definition 2. We define process context closure and term context closure as:

$$
\begin{aligned}
(\mathcal{E} ; r)^{\circ}= & \{(C[\widetilde{M}], C[\widetilde{N}]) \mid \\
& b n(C) \cap f n(\widetilde{M}, \widetilde{N})=\emptyset, f n(C) \subseteq r,(\widetilde{M}, \widetilde{N}) \in \mathcal{E}\} \\
(\mathcal{E} ; r)^{\star}= & \{(D[\widetilde{M}], D[\widetilde{N}]) \mid \\
& b n(D) \cap f n(\widetilde{M}, \widetilde{N})=\emptyset, f n(D) \subseteq r,(\widetilde{M}, \widetilde{N}) \in \mathcal{E}\}
\end{aligned}
$$

The process context closure $(\mathcal{E} ; r)^{\circ}$ intuitively represents the processes that an attacker can craft given some terms and public names. It allows him to create processes using terms $(\widetilde{M}, \widetilde{N})$ from the environment and names from $r$. Capture of names is forbidden, hence the condition on names bound by the context. As this closure uses a context for terms, it will necessarily put its terms in an output prefix or under a run. The term context closure $(\mathcal{E} ; r)^{\star}$ intuitively corresponds to all the terms that the attacker can craft from his knowledge. We point out that these closure operations are monotonic on all their arguments, and thus for any $\mathcal{E}$ and $r,(\mathcal{E} ; r)^{\star}$ includes the identity $(\emptyset ; r)^{\star}$.

A few extra notations are used in this paper. We define the weak transitions $\stackrel{\tau}{\Rightarrow}$ (or $\Rightarrow$ ) as the reflexive transitive closure of $\stackrel{\tau}{\rightarrow}$ (or $\rightarrow$ ), and $\stackrel{\alpha}{\Rightarrow}$ as $\stackrel{\tau}{\Rightarrow} \stackrel{\alpha}{\rightarrow} \stackrel{\tau}{\Rightarrow}$ for any $\alpha \neq \tau$. Finally, we write $a \oplus b$ to express the union $\{a\} \cup b$.

We now formally define environmental bisimulations (a subset of environmental relations):

Definition 3. $\mathcal{X}$ is an environmental bisimulation if for all $(s \vdash P) \mathcal{X}_{\mathcal{E} ; r}(t \vdash Q)$,

1) if $s \vdash P \stackrel{\tau}{\rightarrow} s^{\prime} \vdash P^{\prime}$ then there is $t^{\prime} \vdash Q^{\prime}$ such that $t \vdash Q \stackrel{\tau}{\Rightarrow} t^{\prime} \vdash Q^{\prime}$ and $\left(s^{\prime} \vdash P^{\prime}\right) \mathcal{X}_{\mathcal{E} ; r}\left(t^{\prime} \vdash Q^{\prime}\right)$,

2) if $s \vdash P \stackrel{a(M)}{\longrightarrow} s \vdash P^{\prime}$ with $a \in r$ and $(M, N) \in(\mathcal{E} ; r)^{\star}$, then there is $t^{\prime} \vdash Q^{\prime}$ such that $t \vdash Q \stackrel{a(N)}{\Longrightarrow} t^{\prime} \vdash Q^{\prime}$ and $\left(s \vdash P^{\prime}\right) \mathcal{X}_{\mathcal{E} ; r}\left(t^{\prime} \vdash Q^{\prime}\right)$,

3) if $s \vdash P \stackrel{\bar{a}\langle M\rangle}{\longrightarrow} s \vdash P^{\prime}$ with $a \in r$, then there are $t^{\prime} \vdash Q^{\prime}$ and $N$ such that $t \vdash Q \stackrel{\bar{a}\langle N\rangle}{\longrightarrow} t^{\prime} \vdash Q^{\prime}$ and $\left(s \vdash P^{\prime}\right) \mathcal{X}_{(M, N) \oplus \mathcal{E} ; r}\left(t^{\prime} \vdash Q^{\prime}\right)$,
4) for all $l \in r$ and $\left({ }^{\prime} P_{1},{ }^{\prime} Q_{1}\right) \in \mathcal{E}$, we have $(s \vdash P$ $\left.l\left[P_{1}\right]\right) \mathcal{X}_{\mathcal{E} ; r}\left(t \vdash Q \mid l\left[Q_{1}\right]\right)$,

5) for all $n \notin s \cup t$, we have $(s, n \vdash P) \mathcal{X}_{\mathcal{E} ; r, n}(t, n \vdash Q)$, and

6) the converse of the three first clauses, on Q's transitions.

Clause 1 requires weak reduction closure and is fairly usual; clause 2 requires tested processes in a bisimulation to be able to input on a public channel any related terms that the attacker may create (hence the use of the term context closure), and to have their continuations in the bisimulation; clause 3 enlarges the knowledge of the attacker with terms that were output on a public channel, and requires the continuations to be bisimilar under this new knowledge; clause 4 allows the attacker to spawn and immediately run terms from the environment as processes in parallel to the tested processes (this allows to virtually consider an arbitrary process from the process context closure, while being much more tractable [11, Section 1]); clause 5 means that the attacker can create fresh names at will; finally, clause 6 is just the symmetric of the first three ones.

We remind that, because the set $r$ of names is included in both $s$ and $t$, we know that no name created by $P$ nor $Q$ will clash with $r$; this is why we do not need extra constraints on names like $\left(s^{\prime} \backslash s\right) \cap r=\emptyset$ (in clause 1) and $\left(t^{\prime} \backslash t\right) \cap r=\emptyset$ (in clauses $1,2,3$ ), and why we do not have to require $n \notin r$ in clause 5 . Also, using clause 5 , the attacker can always generate fresh names before creating terms (that will use these new names) for input in clause 2 .

As all the clauses of environmental bisimulations are monotonic on $\mathcal{X}$, the union of all bisimulations exists and is itself an environmental bisimulation. We call it environmental bisimilarity and write it $\sim$. For proving the equivalence of two processes $P$ and $Q$, we show that $(f \vdash P) \sim_{\emptyset ; r}(f \vdash Q)$ for some $r \subseteq f=f n(P, Q)$. It corresponds to equivalence where the attacker can send and receive messages over the public channels $r$ of $P$ and $Q$, but is yet to learn and put any term into the environment. Since $\sim$ is the union of all bisimulations, to prove this equivalence, it suffices to find an environmental bisimulation $\mathcal{X}$ such that $(f \vdash P) \mathcal{X}_{\emptyset ; r}(f \vdash Q)$ with public names $r \subseteq f=f n(P, Q)$.

For improving the practicality of our proof method, we define an up-to context technique. Let us write $\mathcal{X}^{\star}$ for an environmental relation $\mathcal{X}$, such that:

$$
\begin{aligned}
\mathcal{X}^{\star}=\{ & (\mathcal{E}, r, s, P, t, Q)\left|P \equiv P_{0}\right| P_{1}, Q \equiv Q_{0} \mid Q_{1}, \\
& r^{\prime} \cap(s \cup t)=\emptyset, \\
& \left(s, r^{\prime} \vdash P_{0}\right) \mathcal{X}_{\mathcal{E}^{\prime} ; r, r^{\prime}}\left(t, r^{\prime} \vdash Q_{0}\right), \\
& \left.\left(P_{1}, Q_{1}\right) \in\left(\mathcal{E}^{\prime} ; r r^{\prime}\right)^{\circ}, \mathcal{E} \subseteq\left(\mathcal{E}^{\prime} ; r r^{\prime}\right)^{\star}\right\}
\end{aligned}
$$

Even though we call it "up-to context" for simplicity, it is in fact the combination of several up-to techniques: (i) "up-to context" since we allow the spawning of any related processes $\left(P_{1}, Q_{1}\right)$ taken from the process context closure $\left(\mathcal{E}^{\prime} ; r r^{\prime}\right)^{\circ}$ of the knowledge $\mathcal{E}^{\prime}, r r^{\prime}$ in parallel to the tested processes $P_{0}$ and $Q_{0}$ related by $\left(s, r^{\prime} \vdash \cdot\right) \mathcal{X}_{\mathcal{E}^{\prime} ; r r^{\prime}}\left(t, r^{\prime} \vdash \cdot\right)$; (ii) "upto environment" since we allow, through the condition with the term context closure, the use of environments that are 
larger than immediately necessary; (iii) "up-to name creation" since we allow the use of extra new names $r^{\prime}$; and (iv) "up-to structural congruence" since we identify processes structurally congruent to $P_{0} \mid P_{1}$ and $Q_{0} \mid Q_{1}$. This convenient notation allows us to define environmental bisimulations up-to context:

Definition 4. $\mathcal{X}$ is an environmental bisimulation up-to context if for all $(s \vdash P) \mathcal{X}_{\mathcal{E} ; r}(t \vdash Q)$,

1) if $s \vdash P \stackrel{\tau}{\rightarrow} s^{\prime} \vdash P^{\prime}$ then there is $t^{\prime} \vdash Q^{\prime}$ such that $t \vdash Q \stackrel{\tau}{\Rightarrow} t^{\prime} \vdash Q^{\prime}$ and $\left(s^{\prime} \vdash P^{\prime}\right) \mathcal{X}_{\mathcal{E} ; r}^{\star}\left(t^{\prime} \vdash Q^{\prime}\right)$,

2) if $s \vdash P \stackrel{a(M)}{\longrightarrow} s \vdash P^{\prime}$ with $a \in r$ and $(M, N) \in(\mathcal{E} ; r)^{\star}$, then there is $t^{\prime} \vdash Q^{\prime}$ such that $t \vdash Q \stackrel{a(N)}{\longrightarrow} t^{\prime} \vdash Q^{\prime}$ and $\left(s \vdash P^{\prime}\right) \mathcal{X}_{\mathcal{E} ; r}^{\star}\left(t^{\prime} \vdash Q^{\prime}\right)$,

3) if $s \vdash P \stackrel{\bar{a}\langle M\rangle}{\longrightarrow} s \vdash P^{\prime}$ with $a \in r$, then there are $t^{\prime} \vdash Q^{\prime}$ and $N$ such that $t \vdash Q \stackrel{\bar{a}\langle N\rangle}{\Longrightarrow} t^{\prime} \vdash Q^{\prime}$ and $\left(s \vdash P^{\prime}\right) \mathcal{X}_{(M, N) \oplus \mathcal{E} ; r}^{\star}\left(t^{\prime} \vdash Q^{\prime}\right)$,

4) for all $l \in r$ and $\left({ }^{\prime} P_{1}, ' Q_{1}\right) \in \mathcal{E}$, we have $(s \vdash P$ $\left.l\left[P_{1}\right]\right) \mathcal{X}_{\mathcal{E} ; r}^{\star}\left(t \vdash Q \mid l\left[Q_{1}\right]\right)$,

5) for all $n \notin s \cup t$, we have $(s, n \vdash P) \mathcal{X}_{\mathcal{E} ; r, n}(t, n \vdash Q)$, and

6) the converse of the three first clauses, on Q's transitions.

This is basically the same definition as Definition 3 but all the positive instances of $\mathcal{X}$ became $\mathcal{X}^{\star}$ (except in clause 5 for technical reasons). Clause 4 is not a tautology since it spawns terms immediately as processes while the definition of $\mathcal{X}^{\star}$ allows only quoted processes. This distinction between quoted and non-quoted processes enables the use of generic contexts (as in [16], [11]) instead of redex contexts (as in [14]). Similarly to $\sim$, we define environmental bisimilarity up-to context and write it $\simeq$.

Finally, we define environmental similarity $\prec$ and similarity up-to context $\preceq$ by removing the converse conditions from the appropriate definitions.

\section{SOUNDNESS ANd COMPLETENESS OF ENVIRONMENTAL BISIMULATION AND SIMULATION}

We outline here main results and proofs concerning the soundness and completeness of our proof method. More details are found in the appendix [10].

\section{A. Behavioural Equivalences}

We say process $P$ has or exhibits barb $a$ (resp. $\bar{a}$ ), written $P \downarrow_{a}\left(\right.$ resp. $P \downarrow_{\bar{a}}$ ), whenever $P \stackrel{a(\cdot)}{\longrightarrow} \cdot($ resp. $P \stackrel{\bar{a}\langle\cdot\rangle}{\longrightarrow} \cdot$ ). We say process $P$ weakly exhibits barb $\mu$, written $P \Downarrow \mu$, whenever $P \Rightarrow \downarrow \mu$ for a name or a co-name $\mu$.

We can now formally define the equivalence predicates of our language, based on that of [7] (see also [15, Section 2.4.4]) with extensions for name creation.

Definition 5. Reduction-closed barbed equivalence $\approx$ is the largest binary relation on variable-closed configurations, indexed with a set of names $r \subseteq s \cap t$, such that when $s \vdash P \approx_{r} t \vdash Q$,

- $s \vdash P \rightarrow s^{\prime} \vdash P^{\prime}$ implies there are $Q^{\prime}$ and $t^{\prime}$ such that $t \vdash Q \Rightarrow t^{\prime} \vdash Q^{\prime}$ and $s^{\prime} \vdash P^{\prime} \approx_{r} t^{\prime} \vdash Q^{\prime}$,
- $s \vdash P \downarrow_{\mu}$ implies $t \vdash Q \Downarrow_{\mu}$ if $\mu$ or $\bar{\mu}$ is in $r$,

- the converse of the above two on $Q$, and

- for all $R$ with $f n(R) \cap((s \cup t) \backslash r)=\emptyset$, we have $s \cup f n(R) \vdash$ $P\left|R \approx_{r \cup f n(R)} t \cup f n(R) \vdash Q\right| R$.

Note that we parameterised the equivalence with public names $r$. This is necessary for distinguishing the public names from private names that are not known to the attacker and cannot be observed nor used. This explains why the clause on barbs (and its symmetric) only considers barbs in $r$, and why in the last clause private names cannot be free in $R$. However, the free names created by the attacker are public and must thus be added to $r$ for observation, and to $s$ and $t$ to avoid re-creation.

Definition 6. Reduction-closed barbed congruence $\dot{\approx}$ is defined similarly to Definition 5, but replacing $\approx$ with $\dot{\sim}$ and the last clause with: for all $C_{p}$ (context with holes for processes) such that $f n\left(C_{p}\right) \cap((s \cup t) \backslash r)=b n\left(C_{p}\right) \cap f n(P, Q)=\emptyset$, we have $s \cup f n\left(C_{p}\right) \vdash C_{p}[P] \dot{\sim}_{r \cup f n\left(C_{p}\right)} t \cup f n\left(C_{p}\right) \vdash C_{p}[Q]$.

It might be surprising that we consider a "congruence" which cannot capture public names $\left(b n\left(C_{p}\right) \cap f n(P, Q)=\emptyset\right)$, but we argue that this is a reasonable definition. Indeed, free names in our language represent already created constants (private or public) in the compared processes; allowing the capture of names would virtually correspond to allowing inplace changes to the constant values in programs. Even though this may well tell some systems apart-as the attacker wishes to do-we doubt it represents a reasonable way to compare the behaviours of systems in execution contexts (in fact, this rather looks like using a binary editor to tell apart programs by modifying their code).

Definition 7. Reduction-closed barbed approximation $\lesssim$ is defined similarly to Definition 5, but replacing $\approx$ with $\lesssim$ and removing the converse clauses. Respectively, reduction-closed

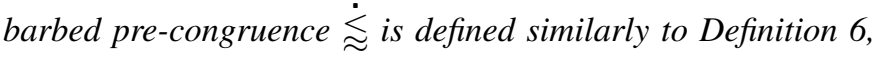
but replacing $\dot{\approx}$ with $\dot{\lesssim}$ and removing the converse clause.

We say $P$ approximates $Q$ if $f \vdash P \lesssim r f \vdash Q$ with some $r \subseteq f=f n(P, Q)$. Intuitively, $P \lesssim Q$ (or $P \lesssim Q$ ) whenever $Q$ can do at least as much as $P$, in parallel with an observer $R$ (or under a non-capturing context $C_{p}$ ).

\section{B. Soundness}

Theorem 1. If $(s \vdash P) \simeq_{\mathcal{E} ; r}(t \vdash Q)$ then $(s \vdash P) \sim_{\mathcal{E} ; r}(t \vdash Q)$.

Outline of proof: knowing $\simeq \subseteq \simeq^{\star}$ by definition, we show that:

1) transitions from $\simeq^{\star}$ lead to $\simeq^{-}$(a superset of $\simeq^{\star}$ called run-erasure [10]),

2) if $(s \vdash P) \simeq_{\mathcal{E}_{;} ;}^{-}(t \vdash Q)$ and $s \vdash P \stackrel{\bar{a}\langle M\rangle}{\longrightarrow} s \vdash P^{\prime}$, then $t \vdash Q \stackrel{\bar{a}\langle N\rangle}{\Longrightarrow} t^{\prime} \vdash Q^{\prime}$ and $\left(s \vdash P^{\prime}\right) \simeq_{(M, N) \oplus \mathcal{E} ; r}^{-}\left(t^{\prime} \vdash Q^{\prime}\right)$, and using this result,

$3) \simeq^{-}$is also closed by input and internal transitions. 
It is then quite easy to show that $\simeq^{-}$verifies all the clauses of environmental bisimulations, that is, $\simeq^{-} \subseteq \sim$.

Corollary 1. If $(f \vdash P) \sim_{\emptyset ; r}(f \vdash Q)$ with $r \subseteq f=f n(P, Q)$, then $f \vdash P \approx_{r} f \vdash Q$.

Outline of proof: we show that $\sim$ is reduction-closed (by definition), that it weakly exhibits the same barbs (by definition of bisimulation, ignoring the continuations after input or output transitions), and that it is preserved by parallel composition of arbitrary processes (that do not use private names) using the up-to context technique (with $\sim \subseteq \simeq$ ).

It is interesting to remark that reduction-closed barbed congruence can easily be shown as follows. Let us define $P \bumpeq_{r} Q$ if $(f \vdash 0) \sim_{\left\{\left({ }^{\prime} P,{ }^{\prime} Q\right)\right\} ; r}(f \vdash 0)$ with $r \subseteq f=f n(P, Q)$. Then:

Theorem 2. If $P \bumpeq_{r} Q$, then $f \vdash P \dot{\approx}_{r} f \vdash Q$ with $r \subseteq f=$ $f n(P, Q)$.

Outline of proof: We first show that a set relating run-erasures of $(C[\widetilde{M}], C[\widetilde{N}])$ for any non-capturing context $C$, with $(s \vdash$ $0) \sim_{\mathcal{E} ; r}(t \vdash 0)$ and $(\widetilde{M}, \widetilde{N}) \in \mathcal{E}$, is reduction-closed and verifies the conditions on barbs of reduction-closed barbed congruence. Then, by $P \bumpeq_{r} Q$, i.e. $(f \vdash 0) \sim_{\left\{\left({ }^{\prime} P,{ }^{\prime} Q\right)\right\} ; r}(f \vdash 0)$ with $r \subseteq f=f n(P, Q)$, we have $f \vdash P \dot{\approx}_{r} f \vdash Q$.

We emphasise that a capturing congruence cannot (and should not) be shown with this method. Omitting ' and run for brevity, we prove this by crafting a counter-example such that $P \bumpeq_{r} Q$ but $P$ and $Q$ are not related by a name-capturing version of $\dot{\approx}$.

Let $P_{1}=a(X) \cdot(X \mid i(Y) \cdot \bar{m}) \mid m \cdot \bar{\omega}$ and $Q_{1}=a(X) \cdot(X \mid$ $i(Y) . Y) \mid m . \bar{\omega}$. We then consider the two processes $P=\nu i . \bar{b}$ $\left\langle P_{1}\right\rangle \mid \bar{c}\langle\bar{a}\langle\bar{i}\langle\bar{m}\rangle\rangle\rangle$ and $Q=\nu i . \bar{b}\left\langle Q_{1}\right\rangle \mid \bar{c}\langle\bar{a}\langle\bar{i}\langle\bar{m}\rangle\rangle\rangle$ which are such that $P \bumpeq_{r} Q$ for $r=\{a, m, b, c, \omega\} . P$ and $Q$ have been designed such that $P$ can exhibit barb $\bar{\omega}$ if it receives anything on private channel $i$, while $Q$ can exhibit barb $\bar{\omega}$ if it receives process $\bar{m}$ on private channel $i$.

By creating name $i$ and then capturing the public name $m$ with a context like $\bar{a}\left\langle\nu m .[]_{1}\right\rangle \mid a(X) .(X \mid X)$, it is possible to reach a state where $\bar{\omega}$ and $\overline{m_{1}} \mid m_{2} \cdot \bar{\omega}$ with private and different $m_{1}$ and $m_{2}$ would be related. However, as only the former process has barb $\bar{\omega}$, the equivalence cannot hold. This shows that comparing processes in a bisimulation environment is not enough to guarantee name-capturing reduction-closed barbed congruence.

It is in fact no problem that two processes in a bisimulation environment are not necessarily related by a name-capturing version of reduction-closed barbed congruence. Indeed, it is consistent with our idea that allowing capture of already created names is not a good basis for a congruence in languages with name creation like $\mathrm{HO} \pi \mathrm{Pn}$.

Soundness also holds for simulations:

Theorem 3. Let $r \subseteq f=f n(P, Q)$. If $(f \vdash P) \preceq_{\emptyset ; r}(f \vdash Q)$, then $(f \vdash P) \lesssim_{r}(f \vdash Q)$. Respectively, if $(f \vdash 0) \preceq_{\left\{\left({ }^{\circ} P,{ }^{\prime} Q\right)\right\} ; r}$ $(f \vdash 0)$, then $(f \vdash P) \dot{\lesssim}_{r}(f \vdash Q)$.
The proof is immediate as our soundness proofs for environmental bisimulations do not use the symmetry condition and therefore can automatically be applied to environmental simulations too.

\section{Completeness}

Theorem 4. If $f \vdash P \approx_{r} f \vdash Q$ with $r \subseteq f=f n(P, Q)$, then $(f \vdash P) \sim_{\emptyset ; r}(f \vdash Q)$.

Outline of proof: we find an environmental bisimulation $\mathcal{X}$ (up-to context) relating reduction-closed barbed equivalent $P$ and $Q$. The trick is to use a parallel product of outputting processes to represent the environment. Roughly,

$$
\left(s \vdash P \mid \prod_{i} l_{i}\left[P_{i}\right]\right) \mathcal{X}_{\mathcal{E} ; r}\left(t \vdash Q \mid \prod_{i} l_{i}\left[Q_{i}\right]\right)
$$

with $\left\{\left({ }^{(} \widetilde{P},{ }^{\prime} \widetilde{Q}\right)\right\} \subseteq \mathcal{E}$ and $\widetilde{l} \in r$ is defined by

$$
\left(s, \widetilde{g} \vdash P \mid \prod_{j} ! \overline{g_{j}}\left\langle{ }^{\prime} P_{j}\right\rangle\right) \approx_{r, \widetilde{g}}\left(t, \widetilde{g} \vdash Q \mid \prod_{j} ! \overline{g_{j}}\left\langle{ }^{\prime} Q_{j}\right\rangle\right)
$$

with $\left\{\left({ }^{(} \widetilde{P},{ }^{\prime} \widetilde{Q}\right)\right\}=\mathcal{E}$. By using the last clause of reductionclosed barbed equivalence, one can create processes that will fetch the necessary ( $\left.\widetilde{P},{ }^{\prime} \widetilde{Q}\right)$ and use them for crafting elements of the context closure $(\mathcal{E} ; r)^{\star}$ needed in the input clause. The spawn clause is satisfied by construction. When accounting for a reaction like $P\left|l_{1}\left[P_{1}\right] \stackrel{\tau}{\rightarrow} P\right| l_{1}\left[P_{1}^{\prime}\right]$, one uses the last clause of reduction-closed barbed equivalence to create a receiver $l_{1}\left[g_{1}(X) . X\right]$, spawns $P_{1}$ (and $Q_{1}$ ) inside this location $l_{1}$, mimics the reduction of $P_{1}$ (and the weak reactions of $Q$ and $Q_{1}$ to $Q^{\prime}$ and $Q_{1}^{\prime}$ ), and then passivates the contents of location $l_{1}$ to put (' $\left.P_{1}^{\prime}, ' Q_{1}^{\prime}\right)$ immediately in the representation of the "environment" under fresh name $g_{j+1}$, giving

$$
\begin{aligned}
& s^{\prime}, \widetilde{g} \vdash P\left|\prod_{j} ! \overline{g_{j}}\left\langle{ }^{\prime} P_{j}\right\rangle\right| ! \overline{g_{j+1}}\left\langle{ }^{\prime} P_{1}^{\prime}\right\rangle \approx_{r, \widetilde{g}} \\
& t^{\prime}, \widetilde{g} \vdash Q^{\prime}\left|\prod_{j} ! \overline{g_{j}}\left\langle{ }^{\prime} Q_{j}\right\rangle\right| ! \overline{g_{j+1}}\left\langle{ }^{\prime} Q_{1}^{\prime}\right\rangle .
\end{aligned}
$$

Therefore, processes $P \mid l_{1}\left[P_{1}^{\prime}\right]$ and $Q^{\prime} \mid l\left[Q_{1}^{\prime}\right]$ are now related as wanted.

Corollary 2. If $f \vdash P \dot{\approx}_{r} f \vdash Q$ with $r \subseteq f=f n(P, Q)$, then $P \bumpeq{ }_{r} Q$.

Outline of proof: by the last clause of reduction-closed barbed congruence, we know that $(f \vdash P) \dot{\approx}_{r}(f \vdash Q)$ implies $(a, f \vdash \bar{a}\langle P\rangle) \dot{\approx}_{a, r}(a, f \vdash \bar{a}\langle Q\rangle)$ for fresh $a$, which itself implies $(a, f \vdash \bar{a}\langle P\rangle) \approx_{a, r}(a, f \vdash \bar{a}\langle Q\rangle)$. By Theorem 4, we thus have $(a, f \vdash \bar{a}\langle P\rangle) \sim_{\emptyset ; a, r}(a, f \vdash \bar{a}\langle Q\rangle)$. We then output to $a$, get $(a, f \vdash 0) \sim_{\left\{\left({ }^{\prime} P,{ }^{\prime} Q\right)\right\} ; a, r}(a, f \vdash 0)$, remove $a$ up-to name creation, and we are done.

We do not know yet whether completeness of simulation holds, since our current proofs rely on the symmetry conditions of the relations.

\section{BISIMILARITY EXAMPLE}

We present an example of equivalence that could not be proven with previous methods, remotely inspired by MapReduce [3] and abstracting the "reduce" part of it. More precisely, we show the bisimilarity between distributed left- and rightfold computations for arbitrary list $l$, associative function $f$, 
and initial value $i$ (the identity element of $f$ ). With car and $c d r$ functions that return the head and tail of a list, we define the "fold servers" as:

$$
\begin{aligned}
L= & ! f l(f, i, l, k) \text {.if null } l \\
& \text { then } \bar{k}\langle i\rangle \text { else } \nu c . \bar{f}\langle f, f i(\text { car } l), c d r l, c\rangle . c(m) . \bar{k}\langle m\rangle \\
R= & ! f r(f, l, i, k) . \text { if } n u l l l \\
& \text { then } \bar{k}\langle i\rangle \text { else } \nu c . \overline{f r}\langle f, c d r l, i, c\rangle . c(m) \cdot \bar{k}\langle f(\operatorname{car} l) m\rangle
\end{aligned}
$$

They are parameterised by (in addition to $f, l$ and $i$ ) a channel $k$ to return their results to clients (although omitted in the preceding sections, we remind our reader that first-order names and constants are easily added to the theory of environmental bisimulation [16]).

We then want to prove equivalent the configurations $a, b, f l \vdash P$ and $a, b, f r \vdash Q$ with public $a$ and $b$, and where:

$$
\begin{aligned}
& P=b(f, l, i, k) \cdot(\overline{f l}\langle f, i, l, k\rangle \mid a[L]) \\
& Q=b(f, l, i, k) \cdot(\overline{f r}\langle f, l, i, k\rangle \mid a[R])
\end{aligned}
$$

To prove their equivalence, we provide a (strong) bisimulation $\mathcal{X}=\mathcal{X}_{1} \cup \mathcal{X}_{2} \cup \mathcal{X}_{3}$ as in Figure 2 (where we use sans-serif fonts to denote Haskell-like functions). We will henceforth use the acronyms LHS and RHS for respectively the lefthand and right-hand sides of the bisimulation, i.e. the tested configurations. In this particular example, the same pairs of transitions verify the bisimulation clauses on both LHS and RHS's transitions; we will therefore only consider the transitions of LHS to avoid redundancy. We now analyse $\mathcal{X}_{1}$, which contains the configurations we want to identify. First, we observe that the set $r$ in $\mathcal{X}_{1}$ contains at least the public names of $P$ and $Q$, as required clause 5 of the bisimulation. Also, since the environment is empty, clause 4 is vacuously satisfied. Then, we consider the transitions, starting with $(f l, r \vdash P) \stackrel{b(f, i, l, k)}{\longrightarrow}(f l, r \vdash \bar{f}\langle f, i, l, k\rangle \mid a[L])$, which is matched by $(f r, r \vdash Q) \stackrel{b(f, i, l, k)}{\longrightarrow}(f r, r \vdash \overline{f r}\langle f, l, i, k\rangle \mid a[R])$ so that membership to $\mathcal{X}_{2}$ is satisfied (by taking $n=1$ and $a_{1}=a$, with up-to environment since $\left.\emptyset \subseteq\left\{\left({ }^{\prime} L,{ }^{\prime} R\right)\right\}\right)$. The name $k$ was added to $r$ in $\mathcal{X}_{1}$ by clause 5 of the bisimulation, and then input by clause 2 .

Then, the elements of $\mathcal{X}_{2}$ must verify the spawn clause since their environment $\left\{\left({ }^{\prime} L,{ }^{\prime} R\right)\right\}$ is not empty; spawning $l[L]$ and $l[R]$ for some $l \in r$ just enlarges the products $\prod_{j=1}^{n} a_{j}[]$ by one element, preserving the membership to $\mathcal{X}_{2}$. Conversely, they can also do a passivation transition (which is a form of higher-order output): a pair $\left({ }^{\prime} L,{ }^{\prime} R\right)$ is necessarily added to the environment (to which it already belongs) and the products shrink by one element; membership to $\mathcal{X}_{2}$ is thus preserved again. Finally, the reaction of $\overline{f l}$ with $L$ (resp. $\overline{f r}$ with $R$ ) gives $\left(\left\{\left({ }^{\circ} L,{ }^{\prime} R\right)\right\}, r, r f l, \prod_{j=1}^{n-1} a_{j}[L]\left|a_{n}\left[L \mid P_{0}\right], r f r, \prod_{j=1}^{n-1} a_{j}[R]\right|\right.$ $\left.a_{n}\left[R \mid Q_{0}\right]\right)$ with $P_{0}=$ if null $l$ then $\bar{k}\langle i\rangle$ else $\nu c . \overline{f l}$ $\langle f, f i($ car $l), c d r l, c\rangle . c(m) \cdot \bar{k}\langle m\rangle$ and $Q_{0}=$ if null $l$ then $\bar{k}$ $\langle i\rangle$ else $\nu c . \overline{f r}\langle f, c d r l, i, c\rangle . c(m) \cdot \bar{k}\langle f($ car $l) m\rangle$, which belongs to $\mathcal{X}_{3}$ up-to environment since $\left\{\left({ }^{\prime} L,{ }^{\prime} R\right)\right\} \subseteq \mathcal{E}$.

We now show that $\mathcal{X}_{3}$ satisfies the clauses of environmental bisimulation. Because all locations $a_{j}$ in $\mathcal{X}_{3}$ are public and may thus lead to passivation, all $P_{j}, Q_{j}$ must be in the

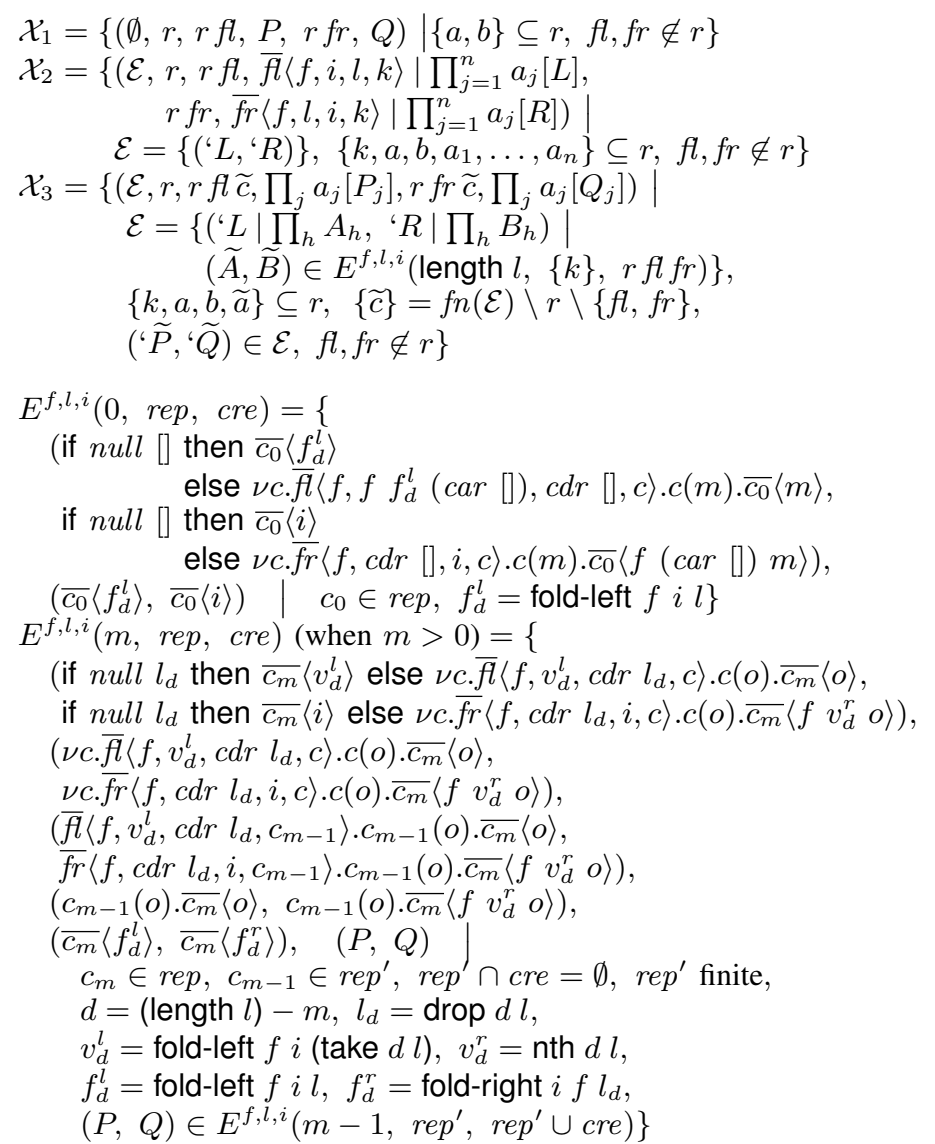

Fig. 2. The partitions of the bisimulation $\mathcal{X}$

environment (by clause 3 of the bisimulation); conversely, all terms from the environment must be spawnable an arbitrary number of times as located processes (by clause 4). It is straightforward to verify that $\mathcal{X}_{3}$ satisfies these constraints by definition. Respect of clause 5 is also immediate to verify.

We then remark that $\mathcal{X}_{3}$ contains only locations $a_{j}$ hosting elements of $\mathcal{E}$, i.e. the fold servers $L$ and $R$ in parallel with their related continuations $A_{h}$ and $B_{h}$ (if any). Therefore, in order to analyse the other transitions of elements of $\mathcal{X}_{3}$, we morally just have to consider the transitions of the elements of $\mathcal{E}$, i.e. the servers and their continuations. Those continuations are members of the set $E^{f, l, i}$ (length $l,\{k\}, r f l f r$ ), where $E$ is a recursive function parametric in several values (see Figure 2). Concretely, the fixed parameters are the function $f$ to fold, the initial list $l$ and the initial value $i$. The varying parameters are the number $m$ of elements yet to fold (hence $d=($ length $l)-m$ is the current "depth" in the whole fold), a set rep of channels to return the result of the current recursive call, and a set cre of already created names.

Let us therefore consider first the transitions of $P_{d}=$ if null $l_{d}$ then $\overline{c_{m}}\left\langle v_{d}^{l}\right\rangle$ else $\ldots$ and related $Q_{d}=$ if $n$ ull $l_{d}$ then $\overline{c_{m}}\langle i\rangle$ else $\ldots$ in some $E_{m}=E^{f, l, i}(m$, rep , cre). If this is the "last recursive call," i.e. $m=0$, then $l_{d}=$ [] and $P_{d} \stackrel{\tau}{\rightarrow} \overline{c_{m}}\left\langle f_{d}^{l}\right\rangle$ where $f_{d}^{l}=$ fold-left $f i l$ is the final value (by definition of left fold), and $Q_{d} \stackrel{\tau}{\rightarrow} \overline{c_{m}}\langle i\rangle$ (by definition too). Since $\overline{c_{m}}\left\langle f_{d}^{l}\right\rangle$ and $\overline{c_{m}}\langle i\rangle$ are also related by $E_{m}$, these 
transitions preserve membership to $\mathcal{X}_{3}$.

If $m>0$ (i.e. the recursive call is not the last one), then the else branches are taken in both processes, giving $P_{d}^{\prime}=\nu c . \overline{f l}\langle f$, $\left.v_{d}^{l}, c d r l_{d}, c\right\rangle . c(o) . \overline{c_{m}}\langle o\rangle$ in LHS and $Q_{d}^{\prime}=\nu c . \overline{f r}\left\langle f, c d r l_{d}, i\right.$, $\left.c\rangle . c(o) \cdot \overline{c_{m}}\left\langle f v_{d}^{r} o\right\rangle\right)$ in RHS, still preserving membership to $\mathcal{X}_{3}$ since $\left(P_{d}^{\prime}, Q_{d}^{\prime}\right) \in E_{m}$.

Then, $P_{d}^{\prime}$ and $Q_{d}^{\prime}$ can both create a name $c_{m-1}$ to become $P_{d}^{\prime \prime}=\bar{f}\left\langle f, v_{d}^{l}, c d r l_{d}, c_{m-1}\right\rangle \cdot c_{m-1}(o) . \overline{c_{m}}\langle o\rangle$ and $Q_{d}^{\prime \prime}=\overline{f r}\langle f$, $\left.\left.c d r l_{d}, i, c_{m-1}\right\rangle \cdot c_{m-1}(o) . \overline{c_{m}}\left\langle f v_{d}^{r} o\right\rangle\right)$, provided $c_{m-1}$ has not already been created, i.e. that $c_{m-1}$ is not in cre. But remember that, by definition of $\mathcal{X}_{3}$ (that verifies clause 4 of the bisimulation), continuations $P_{d}^{\prime}$ and $Q_{d}^{\prime}$ can be spawned several times since they belong to $\mathcal{E}$ (along with $L$ and $R$ ), and that each copy can thus create its own name $c_{m-1}$. Therefore, we must relate several $\left(P_{d}^{\prime \prime}, Q_{d}^{\prime \prime}\right)$ all with their own fresh $c_{m-1}$; the set $r e p^{\prime}$ exactly contains every such $c_{m-1}$. Notice that the names of $r e p^{\prime}$ are free, allowing the definition of $\mathcal{X}_{3}$ to list them as $\{\widetilde{c}\}$, the set of names created by the folds.

Now, in order for $P_{d}^{\prime \prime}$ to do a transition on private name $f$, it must react with a server $L$, modelling a recursive call to the left fold on the rest of the current list. In this case, not only does $P_{d}^{\prime \prime}$ reduce to $P_{d}^{\prime \prime \prime}=c_{m-1}(o) \cdot \overline{c_{m}}\langle o\rangle$, but the replication drawn from $L$ turns into $P_{d+1}=$ if null $l_{d+1}$ then $\overline{c_{m-1}}$ $\left\langle v_{d+1}^{l}\right\rangle$ else ... Naturally, $Q_{d}^{\prime \prime}$ follows as well, giving $Q_{d}^{\prime \prime \prime}=$ $c_{m-1}(o) \cdot \overline{c_{m}}\left\langle f v_{d}^{r} o\right\rangle$ and $Q_{d+1}=$ if null $l_{d+1}$ then $\overline{c_{m-1}}$ $\left\langle v_{d+1}^{r}\right\rangle$ else $\ldots$. Since $\left(P_{d}^{\prime \prime \prime}, Q_{d}^{\prime \prime \prime}\right)$ belongs to $E_{m}$ and $\left(P_{d+1}, Q_{d+1}\right)$ as well (since $E_{m} \supseteq E_{m-1}=E^{f, l, i}(m-1$, $r e p^{\prime}, r e p^{\prime} \cup$ cre) by definition), membership to $\mathcal{X}_{3}$ is still preserved. Notice that any $L$ (and related $R$ ) may be used for the above reaction, even one at a location where some other continuations already exist. Because the $P_{d+1}$ and $Q_{d+1}$ add up next to the server they come from, the definition of $\mathcal{E}$ in $\mathcal{X}_{3}$ contains products of arbitrary length $\prod_{h} A_{h}$ and $\prod_{h} B_{h}$ in parallel with $L$ and $R$. Analysis of the transitions of $P_{d+1}$ and $Q_{d+1}$ is the same as that of the transitions of $P_{d}$ and $Q_{d}$ and needs no further development.

Then, in order for $P_{d}^{\prime \prime \prime}$ to do a transition, it must react on $c_{m-1} \in r e p^{\prime}$. By definition, the only processes that can send on $c_{m-1}$ are $\overline{c_{m-1}}\left\langle f_{d+1}^{l}\right\rangle$ and $\overline{c_{m-1}}\left\langle f_{d+1}^{r}\right\rangle$ in $E_{m-1} \subseteq E_{m}$. Then $P_{d}^{\prime \prime \prime}$ reacts with $\overline{c_{m-1}}\left\langle f_{d+1}^{l}\right\rangle$ and turns into $\overline{c_{m}}\left\langle f_{d}^{l}\right\rangle$ (with $f_{d}^{l}=f_{d+1}^{l}=$ fold-left $\left.f i l\right)$. Similarly, the process $\overline{c_{m-1}}\left\langle f_{d+1}^{r}\right\rangle$ reacts with $Q_{d}^{\prime \prime \prime}$ which then turns into $\overline{c_{m}}\left\langle f v_{d}^{r} f_{d+1}^{r}\right\rangle$, i.e. $\overline{c_{m}}$ $\left\langle f_{d}^{r}\right\rangle$, again preserving membership to $\mathcal{X}_{3}$.

Finally, the processes $\overline{c_{m}}\left\langle f_{d}^{l}\right\rangle$ and $\overline{c_{m}}\left\langle f_{d}^{r}\right\rangle$ may behave differently depending on where $c_{m}$ comes from. If $c_{m}$ is private, then they can react with some continuations $c_{m}(o)$. $\overline{c_{m+1}}\langle o\rangle$ and $\left.c_{m}(o) \cdot \overline{c_{m+1}}\left\langle f v_{r_{d-1}} o\right\rangle\right)$ that are related by $E_{m+1}=E^{f, l, i}\left(m+1\right.$, rep" ${ }^{\prime \prime}$, cre $\backslash$ rep $)$. Then, $\overline{c_{m}}\left\langle f_{d}^{l}\right\rangle$ and $\overline{c_{m}}$ $\left\langle f_{d}^{r}\right\rangle$ both turn into 0 while the continuations' continuations are still related by $E_{m+1}$, like we showed for $E_{m}$ in the previous paragraph. (There are no other reactions between elements of $E_{m}$ and $E_{n}$ with $m \neq n$.) Otherwise, necessarily $c_{m}=k$ by definition of $\mathcal{X}_{3}$ and thus, by definition of $E$, the same value fold-left $f i l=$ fold-right $f l i$ is output to public channel $k$.

This concludes our proof that elements of $\mathcal{X}=\mathcal{X}_{1} \cup \mathcal{X}_{2} \cup$ $\mathcal{X}_{3}$ satisfy the clauses of environmental bisimulations (up-to environment), and thus that $a, b, f \vdash P$ and $a, b, f r \vdash Q$ are bisimilar with public names $a$ and $b$.

\section{NON-BISIMILAR EXAMPLES}

\section{A. Non-Bisimilarity Due to Different Internal Reactions}

In the introduction, we gave an example of perhaps surprising (but rational) non-bisimilarity between located processes $l[\nu a . \nu b . P]$ and $l[\nu b . \nu a . P]$. A possibly even more surprising example would be the following:

$\left\{l_{1}, \omega\right\} \vdash l_{1}[\nu a .(a \mid \bar{a} \cdot \bar{\omega})] \not \varkappa_{\left\{\omega, l_{1}\right\}}\left\{l_{1}, \omega\right\} \vdash l_{1}[\nu a . \nu b .(a . b \mid \bar{a} \cdot \bar{b} \cdot \bar{\omega})]$

To see why these configurations are not bisimilar, we consider the duplication of the located processes after the name creations; for $s=\left\{l_{1}, l_{2}, a, \omega\right\}$, we have:

$$
\begin{aligned}
& s \vdash l_{1}[a \mid \bar{a} \cdot \bar{\omega}] \mid l_{2}[a \mid \bar{a} \cdot \bar{\omega}] \not_{\left\{\omega, l_{1}, l_{2}\right\}} \\
& s, b \vdash l_{1}[a . b \mid \bar{a} . \bar{b} \cdot \bar{\omega}] \mid l_{2}[a . b \mid \bar{a} \cdot \bar{b} \cdot \bar{\omega}]
\end{aligned}
$$

Consider now the transition of the right-hand side:

$s \vdash l_{1}[a . b \mid \bar{a} . \bar{b} . \bar{\omega}]\left|l_{2}[a . b \mid \bar{a} . \bar{b} . \bar{\omega}] \stackrel{\tau}{\rightarrow} s \vdash l_{1}[b \mid \bar{a} . \bar{b} \cdot \bar{\omega}]\right| l_{2}[a . b \mid \bar{b} \cdot \bar{\omega}]$

To match, the left-hand side may do a weak transition to one of the six following processes:

$$
\begin{array}{lll}
l_{1}[\bar{\omega}] \mid l_{2}[\bar{\omega}] & l_{1}[a \mid \bar{a} \cdot \bar{\omega}] \mid l_{2}[\bar{\omega}] & l_{1}[\bar{\omega}] \mid l_{2}[a \mid \bar{a} \cdot \bar{\omega}] \\
l_{1}[a \mid \bar{\omega}] \mid l_{2}[\bar{a} \cdot \bar{\omega}] & l_{1}[\bar{a} \cdot \bar{\omega}] \mid l_{2}[a \mid \bar{\omega}] & l_{1}[a \mid \bar{a} \cdot \bar{\omega}] \mid l_{2}[a \mid \bar{a} \cdot \bar{\omega}]
\end{array}
$$

with created names $s$. Suppose that the attacker then passivates $l_{1}$ on the right-hand side:

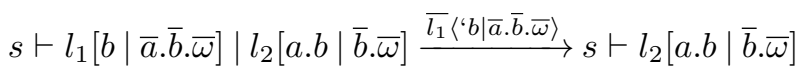

The resulting process is stuck, so because of the symmetry of bisimulations, the left-hand side must be able to passivate $l_{1}$ and become stuck too. The only way to achieve this is necessarily by doing a transition $\stackrel{\overline{l_{1}}\langle a \mid \bar{\omega}\rangle}{=} l_{1}, l_{2}, a, \omega \vdash l_{2}[\bar{a} \cdot \bar{\omega}]$. The attacker can then passivate the contents of $l_{2}$ in both sides of the bisimulation, and be left with processes 0 . Now, he can spawn back what was output during the passivation of $l_{1}$, and we thus have:

$$
s \vdash l_{1}[a \mid \bar{\omega}] \not \not_{\left\{\omega, l_{1}, l_{2}\right\}} s, b \vdash l_{1}[b \mid \bar{a} . \bar{b} \cdot \bar{\omega}]
$$

Obviously, the right-hand side is stuck, but the left one can exhibit $\bar{\omega}$, thus proving that the two processes are not bisimilar.

\section{B. Non-Bisimilarity Due to Different Number of Locations Used}

In Section V, we compared processes that recurse the same number of times and built bisimulations relating these processes such that whenever a process uses a location so does the other. We illustrate now that, in our distributed setting, the number of locations used does matter to draw some bisimilarity results.

Let us consider two implementations of the power function pow $(a, b)=a^{b}$, one of linear complexity, and the other of logarithmic complexity, as shown in Figure 3. Suppose that we want to replace the linear implementation $P$ by the logarithmic one $Q$ in a distributed system, and to check if 


$$
\begin{aligned}
& P=! p o w_{l i n}(a, b, k) \text {.if } b=0 \\
& \text { then } \bar{k}\langle 1\rangle \text { else } \nu c . \overline{p o w_{\text {lin }}}\langle a, b-1, c\rangle . c(m) . \bar{k}\langle a \times m\rangle \\
& Q=\text { ! pow } w_{\log }(a, b, k) \text {.if } b=0 \\
& \text { then } \bar{k}\langle 1\rangle \text { else if } b \% 2=0 \text { then } \overline{p o w_{l o g}}\langle a \times a, b \div 2, k\rangle \\
& \text { else } \nu c . \overline{p o w_{l o g}}\langle a, b-1, c\rangle . c(m) . \bar{k}\langle a \times m\rangle
\end{aligned}
$$

Fig. 3. Linear and logarithmic power functions

there will be no visible difference. We could model those systems as $l[P] \mid \overline{p o w_{l i n}}\langle a, b, k\rangle$ and $l[Q] \mid \overline{p o w l o g}\langle a, b, k\rangle$ for integers $a, b$ and public names $l, k$, and show their equivalence using our proof technique, trying to build a bisimulation $\mathcal{X}$ relating them, starting with $\left(\emptyset, l k, l k\right.$ pow $_{l i n}, l[P] \mid$ $\overline{\text { pow }_{\text {lin }}}\langle a, b, k\rangle, l k$ pow $\left._{\text {log }}, l[Q] \mid \overline{p_{0} w_{l o g}}\langle a, b, k\rangle\right) \in \mathcal{X}$. We consider the situation where location $l$ is passivated and then spawned $b$ times: $\left(\emptyset, l k, l k\right.$ pow $_{\text {lin }}, \prod_{i=1}^{b} l_{i}[P] \mid \overline{\text { powlin }}$ $\langle a, b, k\rangle, l k$ pow $\left.w_{\text {log }}, \prod_{i=1}^{b} l_{i}[Q] \mid \overline{p^{\prime} w_{\text {log }}}\langle a, b, k\rangle\right) \in \mathcal{X}$. Let us consider now the state where the linear version has unfolded all the $b$ recursive calls across $l_{1}, \ldots, l_{b}$, like: $l_{1}\left[P \mid c_{1}(x) . \bar{k}\right.$ $\langle a \times x\rangle]\left|l_{2}\left[P \mid c_{2}(x) . \overline{c_{1}}\langle a \times x\rangle\right]\right| \ldots \mid l_{b}\left[P \mid \overline{c_{b-1}}\langle 1\rangle\right]$. Similarly, the logarithmic version should (somehow) follow weakly: $l_{1}[Q \mid \ldots]\left|l_{2}[Q \mid \ldots]\right| \ldots \mid l_{b}[Q \mid \ldots]$. Suppose now that the attacker passivates the location $l_{1}$ that contains $c_{1}(x) \cdot \bar{k}$ $\langle a \times x\rangle$, so that the left hand-side of the bisimulation can no longer return its result. Then, by the definition of bisimilarity, the logarithmic implementation too must not return a value if $l_{1}$ is passivated. The attacker may now spawn back the passivated contents of $l_{1}$, and then repeat the same passivation test on each of $l_{2}, l_{3} \ldots$. In the end, we know that more than $\log (b)$ locations were necessary for the recursive calls of the logarithmic version of the power function, which is impossible by design since this implementation can do at most $\log (b)$ recursive calls. The two systems thus cannot be bisimilar.

\section{Mutual Simulation}

Although bisimilarity does not hold, we prove that the distributed linear power function approximates the logarithmic one by crafting a simulation relating them. We build the simulation $\mathcal{Y}=\mathcal{Y}_{1} \cup \mathcal{Y}_{2}$ as in Figure 4, in a manner very similar to that of Section V. Our explanations below will thus focus on main differences from Section V. First, we recall that, because we consider (weak) simulations, we require that transitions by LHS be (weakly) matched by transitions on RHS, but not the converse. Thus, in this example, while the simulation $\mathcal{Y}$ needs to keep in LHS all the intermediate states of the linear power calculation, it suffices to keep in RHS only the initial and final states of the logarithmic power calculation.

The initial states are related by $\mathcal{Y}_{1}$. As far as intermediate states are concerned, we decide that the processes of LHS that can do an observable action (i.e. an output to $k$ ) be related to processes in RHS able to do the same action, so as to guarantee satisfaction of the output clause of simulation. Therefore, we define $\mathcal{Y}_{2}$ such that subprocesses of LHS that have $k$ free in them (i.e. continuations of the initial call to $p o w_{l i n}$ ) are related to $\bar{k}\left\langle a^{b}\right\rangle$ on RHS (i.e. final state of the call to $p o w_{\text {log }}$ ), and that other subprocesses of LHS are related to 0 on RHS.

$$
\begin{aligned}
& \mathcal{Y}_{1}=\left\{\left(\mathcal{E}, r, r \text { pow } w_{\text {lin }}, \prod_{i}^{n} l_{i}[P] \mid \overline{p o w_{\text {lin }}}\langle a, b, k\rangle,\right.\right. \\
& \left.r \text { pow } w_{\text {log }}, \prod_{i}^{n} l_{i}[Q] \mid \overline{p_{\text {ow }}}\langle a, b, k\rangle\right) \mid \\
& \left.k, \tilde{l} \in r, \mathcal{E} \subseteq\left\{\left({ }^{\prime} P, ' Q\right)\right\}\right\} \\
& \mathcal{Y}_{2}=\left\{\left(\mathcal{E}, r, \widetilde{c} r \text { powlin }, \prod_{i} l_{i}\left[P_{i}\right], \widetilde{d} r \text { pow } \text { pog }_{\text {log }}, \prod_{i} l_{i}\left[Q_{i}\right]\right)\right. \\
& \mathcal{E}=\left\{\left(P\left|\prod_{h} A_{h}, Q\right| \prod_{h} B_{h}\right) \mid\right. \\
& \left.(\widetilde{A}, \widetilde{B}) \in E^{a, b}\left(b,\{k\}, \text { pow }_{\text {lin }} \text { pow } w_{\text {log }} r\right)\right\} \text {, } \\
& \left({ }^{(} \widetilde{P}, \cdot \widetilde{Q}\right) \in \mathcal{E}, k, \widetilde{l} \in r, \widetilde{c}=f n(\mathcal{E} .1) \backslash r \backslash\left\{\text { pow }_{\text {lin }}\right\}, \\
& \widetilde{d} \cap r \backslash\left\{\text { pow }_{\log }\right\}=\emptyset,|\widetilde{d}|=\operatorname{depth}(b), \\
& \operatorname{depth}(x)= \begin{cases}0 & \text { if } x=0 \\
\operatorname{depth}(x / 2) & \text { if } x \% 2=0 \\
1+\operatorname{depth}(x-1) & \text { otherwise }\}\end{cases} \\
& E^{a, b}(0, r e p, \text { cre })=\{ \\
& \text { (if } 0=0 \text { then } \overline{c_{0}}\langle 1\rangle \text { else } \ldots, R \text { ), }\left(\overline{c_{0}}\langle 1\rangle, R\right. \text { ) } \\
& \left.c_{0} \in \operatorname{rep}, R=\overline{c_{0}}\langle 1\rangle \text { if } b=0 \text {, otherwise } R=0\right\} \\
& E^{a, b}(m, \text { rep }, \text { cre })(\text { when } m>0)=\{ \\
& \text { (if } m=0 \text { then } \ldots \text { else } \nu c \cdot \overline{p o w} \text { lin }\langle a, m-1, c\rangle \cdot c(o) \cdot \overline{c_{m}}\langle a \times o\rangle, R \text { ), } \\
& \left(\nu c . \overline{p o w l i n}\langle a, m-1, c\rangle . c(o) \cdot \overline{c_{m}}\langle a \times o\rangle, R\right) \text {, } \\
& \left(\overline{p o w} \text { lin }\left\langle a, m-1, c_{m-1}\right\rangle \cdot c_{m-1}(o) \cdot \overline{c_{m}}\langle a \times o\rangle, R\right), \\
& \left(c_{m-1}(o) \cdot \overline{c_{m}}\langle a \times o\rangle, R\right),\left(\overline{c_{m}}\left\langle a^{m}\right\rangle, R\right),\left(P_{m-1}, 0\right) \\
& c_{m} \in r e p, c_{m-1} \in r e p^{\prime}, r e p^{\prime} \cap c r e=\emptyset \text {, rep finite, } \\
& P_{m-1} \in E^{a, b}\left(m-1, r e p^{\prime}, r e p^{\prime} \cup c r e\right) \text {, } \\
& \left.R=\overline{c_{m}}\left\langle a^{b}\right\rangle \text { if } m=b \text {, otherwise } R=0\right\}
\end{aligned}
$$

Fig. 4. Simulation $\mathcal{Y}=\mathcal{Y}_{1} \cup \mathcal{Y}_{2}$ between linear and logarithmic power functions

We now show that the set $\mathcal{Y}=\mathcal{Y}_{1} \cup \mathcal{Y}_{2}$ is a weak environmental simulation. We start with $\mathcal{Y}_{1}$ that relates (for $n=1$ and $l_{1}=l$ ) the processes we want to prove related: $l k$ pow $_{\text {lin }} \vdash l[P] \mid \overline{p o w}_{\text {lin }}\langle a, b, k\rangle$ and $l k$ pow $w_{\text {log }} \vdash l[Q] \mid \overline{p o w_{\text {log }}}$ $\langle a, b, k\rangle)$. Suppose that the client $\overline{p o w_{\text {lin }}}\langle a, b, k\rangle$ of LHS reacts with located server $l_{i}[P]$, leaving a process $P_{b}=$ (if $b=$ 0 then $\bar{k}\langle 1\rangle$ else $\nu c \cdot \overline{p o w}$ lin $\langle a, b-1, c\rangle . c(m) . \bar{k}\langle a \times m\rangle)$ at $l_{i}$. RHS can follow by reacting weakly (doing all calculations on the spot) with $l[Q]$, leaving process $l_{i}\left[\bar{k}\left\langle a^{b}\right\rangle\right]$. The rests are related by $\mathcal{Y}_{2}$ up-to environment since $\left\{\left({ }^{\prime} P,{ }^{\prime} Q\right),\left({ }^{\prime} P\left|P_{b},{ }^{\prime} Q\right| \bar{k}\right.\right.$ $\left.\left.\left\langle a^{b}\right\rangle\right)\right\} \subseteq \mathcal{E}$.

Then, if $b=0, P_{b}$ reduces to $\bar{k}\langle 1\rangle$, while RHS's $\bar{k}$ $\left\langle a^{b}\right\rangle=\bar{k}\langle 1\rangle$ follows weakly by not doing any transition. The continuations $\bar{k}\langle 1\rangle$ and $\bar{k}\langle 1\rangle$ preserve membership to $\mathcal{Y}_{2}$. Moreover, if LHS's $\bar{k}\langle 1\rangle$ outputs 1 to $k$, then so can RHS's $\bar{k}$ $\langle 1\rangle$ as expected.

Otherwise, if $b>0, P_{b}$ can reduce successively to $P_{b}^{\prime}=\nu c$. $\overline{p o w_{\text {lin }}}\langle a, b-1, c\rangle \ldots$ and to $P_{b}^{\prime \prime}=\overline{p o w_{\text {lin }}}\left\langle a, b-1, c_{b}\right\rangle \ldots$ In RHS, $\bar{k}\left\langle a^{b}\right\rangle$ follows both transitions weakly, still preserving membership to $\mathcal{Y}_{2}$.

Then $P_{b}^{\prime \prime}$ can react with a server $P: P_{b}^{\prime \prime}$ becomes $P_{b}^{\prime \prime \prime}=$ $c_{b-1}(o) . \bar{k}\langle a \times o\rangle, P$ becomes $P\left|P_{b-1}=P\right|$ if $(b-1)=$ 0 then $\overline{c_{b-1}}\langle 1\rangle$ else $\nu c \cdot \overline{p o w_{\text {lin }}}\langle a, b-2, c\rangle . c(m) \cdot \overline{c_{b-1}}\langle a \times m\rangle$, and $\bar{k}\left\langle a^{b}\right\rangle$ follows weakly. For example, we have $l_{1}\left[P \mid P_{b}^{\prime \prime}\right] \mid$ $l_{2}[P] \stackrel{\tau}{\rightarrow} l_{1}\left[P \mid P_{b}^{\prime \prime \prime}\right] \mid l_{2}\left[P \mid P_{b-1}\right]$ in LHS, and $l_{1}[Q \mid \bar{k}$ $\left.\left\langle a^{b}\right\rangle\right]\left|l_{2}[Q] \stackrel{\tau}{\rightarrow} l_{1}\left[Q \mid \bar{k}\left\langle a^{b}\right\rangle\right]\right| l_{2}[Q \mid 0]$ in RHS. It results from the above that $P_{b}^{\prime \prime \prime}$ is related to $\bar{k}\left\langle a^{b}\right\rangle$, and $P_{b-1}$ to 0 since no process was added in RHS by the weak transition. Membership to $\mathcal{Y}_{2}$ is still satisfied.

We skip the analysis of transitions of $P_{b-1}$, as it similar to that of transitions of $P_{b}$.

If $P_{b}^{\prime \prime \prime}$ inputs on $c_{b-1}$, it becomes $P_{b}^{\prime \prime \prime \prime}=\bar{k}\left\langle a^{b}\right\rangle$ because the only output to $c_{b-1}$ comes from $\overline{c_{b-1}}\left\langle a^{b-1}\right\rangle$ in 
$E^{a, b}\left(b-1, r e p^{\prime}, r e p^{\prime} \cup c r e\right)$. RHS follows weakly, still giving $\bar{k}\left\langle a^{b}\right\rangle$, and preserving membership to $\mathcal{Y}_{2}$. Finally, output of $a^{b}$ to $k$ by both processes can happen, satisfying the simulation's output clause.

This concludes our proof that elements of $\mathcal{Y}=\mathcal{Y}_{1} \cup \mathcal{Y}_{2}$ satisfy the clauses of environmental simulation (up-to environment), and thus that the distributed linear algorithm approximates the logarithmic one.

With the same approach, we may easily show that the linear algorithm simulates the logarithmic one as well (the previous simulation proof does not depend on the number of reduction steps on the left-hand side). This means that the two algorithms simulate each other even though they are not bisimilar, supporting the usefulness of mutual simulation in higher-order distribution.

By the same reasoning, the non-bisimilar processes in the introduction and Section VI-A can also be shown to be mutually similar.

\section{DISCUSSIONS}

We defined $\mathrm{HO} \pi \mathrm{Pn}$, the higher-order distributed $\pi$-calculus with passivation and name creation, and developed its equivalence and inequivalence theories. Although many of the inequivalences may have been counter-intuitive, we emphasise that they are rational in hindsight and reflect the reality of nonlinear higher-order distribution (not necessarily passivation but also duplication in general; cf. [2]).

Recently studied higher-order distributed process calculi include the Kell calculus [18], Homer [6] and the higher-order $\pi$-calculus with passivation ( $\mathrm{HO} \pi \mathrm{P})$ [8]. They are extensions of the $\pi$-calculus with the communication of processes and their execution inside locations, and all have name restriction semantics. Other distributed process calculi such as Ambients [1] and Dpi [4] identify name creation and name restriction semantics, but are not higher-order in our sense (of passing processes through channels).

Research on the Kell calculus and Homer led to defining sound and complete context bisimulations [13]. However, they critically rely on universal quantification on contexts and are almost as hard as reduction-closed barbed equivalence as proof methods. Later, Lenglet et al. [8] focused on $\mathrm{HO} \pi \mathrm{P}$, a calculus simpler than both the Kell calculus and Homer. In addition to sound and complete context bisimulations, they provided more practical normal bisimulations [13] that are sound and complete in the absence of name restriction but are unsound otherwise. Also for $\mathrm{HO} \pi \mathrm{P}$, Piérard and Sumii defined a sound but incomplete environmental bisimulation proof technique [11] with strong constraints (the environments could not contain any restriction operator nor higher-order inputs). Even though non-trivial equivalences of processes which could not be realistically proven with context bisimulations can be proven with this technique, the constraints have a severe impact on the variety of processes that can be considered.

The simplicity of $\mathrm{HO} \pi \mathrm{Pn}$, notably the transparency of locations, does not offer enough control over the communications between processes, and therefore hinders natural modelling of real systems where processes cannot freely interact with one another. Such systems can be modelled with non-transparent locations [18], [6], e.g. locations that only allow communications between processes from the same level or one level above/below. Moreover, passivation in $\mathrm{HO} \pi \mathrm{Pn}$ unifies failure, migration, and duplication as higher-order outputs, therefore mixing different behaviours. Even though identifying them keeps the model simple, their distinction may enable a more realistic modelling of higher-order distributed systems.

\section{REFERENCES}

[1] L. Cardelli and A. D. Gordon. Mobile ambients. In Foundations of Software Science and Computation Structures, volume 1378 of Lecture Notes in Computer Science, pages 140-155. Springer, 1998.

[2] G. Castagna, J. Vitek, and F. Z. Nardelli. The Seal Calculus. Information and Computation, 201(1):1-54, 2005.

[3] J. Dean and S. Ghemawat. Mapreduce: Simplified data processing on large clusters. In Proceedings of the 6th conference on Symposium on Operating Systems Design and Implementation, volume 6, pages 137150. USENIX Association, 2004.

[4] M. Hennessy and J. Riely. Resource access control in systems of mobile agents. Information and Computation, 173:82-120, 1998.

[5] Hewlett-Packard. Live migration across data centers and disaster tolerant virtualization architecture with HP storageworks cluster extension and Microsoft Hyper-V. http://h20195.www2.hp.com/V2/GetPDF.aspx/ 4AA2-6905ENW.pdf.

[6] T. Hildebrandt, J. C. Godskesen, and M. Bundgaard. Bisimulation congruences for Homer: a calculus of higher-order mobile embedded resources. Technical Report TR-2004-52, IT University of Copenhagen, 2004.

[7] K. Honda and N. Yoshida. On reduction-based process semantics. Theoretical Computer Science, 151(2):437-486, 1995.

[8] S. Lenglet, A. Schmitt, and J.-B. Stefani. Normal bisimulations in calculi with passivation. In Foundations of Software Science and Computational Structures, volume 5504 of Lecture Notes in Computer Science, pages 257-271. Springer, 2009.

[9] R. Milner. Communicating and Mobile Systems: the Pi-Calculus. Cambridge University Press, 1999.

[10] A. Piérard and E. Sumii. Appendix to a higher-order distributed calculus with name creation. http://www.kb.ecei.tohoku.ac.jp/ adrien/ pubs/AppendixCreation.pdf.

[11] A. Piérard and E. Sumii. Sound bisimulations for higher-order distributed process calculus. In Foundations of Software Science and Computational Structures, volume 6604 of Lecture Notes in Computer Science, pages 123-137. Springer, 2011.

[12] D. Sangiorgi. Expressing Mobility in Process Algebras: First-Order and Higher-Order Paradigms. PhD thesis, University of Edinburgh, 1992.

[13] D. Sangiorgi. Bisimulation for higher-order process calculi. Information and Computation, 131:141-178, 1996.

[14] D. Sangiorgi, N. Kobayashi, and E. Sumii. Environmental bisimulations for higher-order languages. ACM Transactions on Programming Languages and Systems, 33:5:1-5:69, 2011.

[15] D. Sangiorgi and D. Walker. The $\pi$-calculus: a Theory of Mobile Processes. Cambridge University Press, 2001.

[16] N. Sato and E. Sumii. The higher-order, call-by-value applied picalculus. In Asian Symposium on Programming Languages and Systems, volume 5904 of Lecture Notes in Computer Science, pages 311-326. Springer, 2009.

[17] D. Schmidt and P. Dhawan. Live migration with Xen virtualization software. http://www.dell.com/downloads/global/power/ ps2q06-20050322-Schmidt-OE.pdf.

[18] A. Schmitt and J.-B. Stefani. The Kell calculus: A family of higherorder distributed process calculi. In Global Computing, volume 3267 of Lecture Notes in Computer Science, pages 146-178. Springer, 2004.

[19] I. Stark. Names and Higher-Order Functions. PhD thesis, University of Cambridge, 1994. Also available as Technical Report 363, University of Cambridge Computer Laboratory.

[20] E. Sumii and B. C. Pierce. A bisimulation for dynamic sealing. Theoretical Computer Science, 375(1-3):169-192, 2007.

[21] E. Sumii and B. C. Pierce. A bisimulation for type abstraction and recursion. Journal of the ACM, 54:1-43, 2007. 


\section{A Higher-order $\pi$-calculus with passivation and name creation}

\section{Syntax}

The syntax of $\mathrm{HO} \pi \mathrm{Pn}$ processes $P, Q$ is given by the following grammar:

$$
\begin{aligned}
& P, Q::=0|a(X) . P| \bar{a}\langle M\rangle . P|(P \mid P)| a[P]|\nu a . P| ! P \mid \operatorname{run}(M) \\
& M, N::=X \mid ' P
\end{aligned}
$$

We define the functions that returns the free names and free variables respectively as:

$$
\begin{aligned}
& f n(0) \quad=\emptyset \quad f v(0) \quad=\emptyset \\
& f n(a(X) . P)=\{a\} \cup f n(P) \quad f v(a(X) . P)=f v(P) \backslash\{X\} \\
& f n(\bar{a}\langle M\rangle . P)=\{a\} \cup f n(M) \cup f n(P) f v(\bar{a}\langle M\rangle . P)=f v(M) \cup f v(P) \\
& f n\left(P_{1} \mid P_{2}\right)=f n\left(P_{1}\right) \cup f n\left(P_{2}\right) \quad f v\left(P_{1} \mid P_{2}\right)=f v\left(P_{1}\right) \cup f v\left(P_{2}\right) \\
& f n(a[P])=\{a\} \cup f n(P) \quad f v(a[P]) \quad=f v(P) \\
& f n(\nu a . P)=f n(P) \backslash\{a\} \quad f v(\nu a . P)=f v(P) \\
& f n(! P)=f n(P) \quad f v(! P)=f v(P) \\
& f n(\operatorname{run}(M))=f n(M) \quad f v(\operatorname{run}(M))=f v(M) \\
& f n(X)=\emptyset \quad f v(X)=\{X\} \\
& f n\left({ }^{\prime} P\right) \quad=f n(P) \quad f v\left({ }^{\circ} P\right) \quad=f v(P)
\end{aligned}
$$

We conveniently write $f n(X, Y, \ldots, Z)($ resp. $f v(X, Y, \ldots, Z))$ to denote $\bigcup f n(S)$ $S \in\{X, Y, \ldots, Z\}$

(resp. $\bigcup f v(S))$.

$S \in\{X, Y, \ldots, Z\}$

\section{Labelled transitions system}

Definition A.1. [Configuration]

$A$ configuration $s \vdash P$ is the pair of a set $s$ of names and a process $P$ such that $f n(P) \subseteq s$. We casually write $s x$ or $s, x$ for $s \cup\{x\}$ or $s \cup x$ when $x$ is a name or a set of names.

The transitions semantics of $\mathrm{HO} \pi \mathrm{Pn}$ is given by the following labelled transitions system:

$$
\begin{gathered}
\overline{s \vdash a(X) . P \stackrel{a(M)}{\longrightarrow} s \vdash P\{M / X\}} \text { Ho-In } \\
\frac{s \vdash P \stackrel{\alpha}{\rightarrow} s^{\prime} \vdash P^{\prime} \quad\left(s^{\prime} \backslash s\right) \cap f n(Q)=\emptyset}{s \vdash P\left|Q \stackrel{\alpha}{\rightarrow} s^{\prime} \vdash P^{\prime}\right| Q} \text { PAR-L }
\end{gathered}
$$




$$
\begin{gathered}
\frac{s \vdash P \stackrel{\alpha}{\rightarrow} s^{\prime} \vdash P^{\prime} \quad\left(s^{\prime} \backslash s\right) \cap f n(Q)=\emptyset}{s \vdash Q\left|P \stackrel{\alpha}{\rightarrow} s^{\prime} \vdash Q\right| P^{\prime}} \text { PAR-R } \\
\frac{s \vdash P \stackrel{\bar{a}\langle M\rangle}{\rightarrow} s \vdash P^{\prime} \quad s \vdash Q \stackrel{a(M)}{\rightarrow} s \vdash Q^{\prime}}{s \vdash P\left|Q \stackrel{\tau}{\rightarrow} s \vdash P^{\prime}\right| Q^{\prime}} \text { REACT-L } \\
\frac{s \vdash P \stackrel{a(M)}{\rightarrow} s \vdash P^{\prime} \quad s \vdash Q \stackrel{\bar{a}\langle M\rangle}{\rightarrow} s \vdash Q^{\prime}}{s \vdash P\left|Q \stackrel{\tau}{\rightarrow} s \vdash P^{\prime}\right| Q^{\prime}} \text { REACT-R } \\
\frac{s \vdash ! P \mid P \stackrel{\alpha}{\rightarrow} s^{\prime} \vdash P^{\prime}}{s \vdash ! P \stackrel{\alpha}{\rightarrow} s^{\prime} \vdash P^{\prime}} \operatorname{REP} \quad \frac{a \notin s}{s \vdash \nu a . P \stackrel{\tau}{\rightarrow} s, a \vdash P} \text { CREATE } \\
\frac{s \vdash P \stackrel{\alpha}{\rightarrow} s^{\prime} \vdash P}{s \vdash a[P] \stackrel{\alpha}{\rightarrow} s^{\prime} \vdash a\left[P^{\prime}\right]} \operatorname{TRANSP} \frac{s \vdash a[P] \stackrel{\bar{a}\langle\cdot P\rangle}{\rightarrow} s \vdash 0}{s \vdash \operatorname{Pun}\left({ }^{(} P\right) \stackrel{\tau}{\rightarrow} s \vdash P} \text { RUN }
\end{gathered}
$$

with the following function on labels

$$
n(\alpha)= \begin{cases}\emptyset & \text { if } \alpha=\tau \\ \{a\} \cup f n(M) & \text { if } \alpha=a(M) \text { or } \alpha=\bar{a}\langle M\rangle\end{cases}
$$

and the notation $\widetilde{x}$ to denote the sequence $x_{0}, x_{1}, \ldots x_{n}$.

Definition A.2. Structural congruence $\equiv$ is the smallest relation on processes such that:

$$
\begin{aligned}
& \frac{Q \equiv P}{P \equiv Q} \mathrm{~S} \text {-SYM } \quad \frac{P \equiv R \quad R \equiv Q}{P \equiv P} \text { S-REFL } \quad \frac{P \equiv Q}{P \text { TrAnS }} \\
& \overline{P \equiv P \mid 0} \text { S-EMPTY } \overline{P_{1}\left|\left(P_{2} \mid P_{3}\right) \equiv\left(P_{1} \mid P_{2}\right)\right| P_{3}} \text { S-Assoc } \\
& \frac{P \equiv Q}{P_{1}\left|P_{2} \equiv P_{2}\right| P_{1}} \text { S-Commut } \quad \frac{P \equiv Q}{\nu a . P \equiv \nu a \cdot Q} \text { S-Create } \quad \frac{P}{a(X) \cdot P \equiv a(X) \cdot Q} \text { S-IN } \\
& \frac{P_{1} \equiv Q_{1} \quad P_{2} \equiv Q_{2}}{\bar{a}\left\langle{ }^{\prime} P_{1}\right\rangle \cdot P_{2} \equiv \bar{a}\left\langle{ }^{\prime} Q_{1}\right\rangle \cdot Q_{2}} \text { S-OUT } \quad \frac{P \equiv Q}{! P \equiv ! P \mid P} \text { S-REP } \quad \frac{P \equiv ! Q}{! P} \text { S-BANG } \\
& \frac{P_{1} \equiv Q_{1} \quad P_{2} \equiv Q_{2}}{P_{1}\left|P_{2} \equiv Q_{1}\right| Q_{2}} \mathrm{~S} \text {-ComP } \quad \frac{P \equiv Q}{a[P] \equiv a[Q]} \mathrm{S}-\text { LOC } \quad \frac{P \equiv Q}{\operatorname{run}\left({ }^{\prime} P\right) \equiv \operatorname{run}\left({ }^{\prime} Q\right)} \mathrm{S}-\mathrm{RUN}
\end{aligned}
$$

Definition A.3. Structural congruence on labels $\equiv$ is defined by:

$$
\overline{\tau \equiv \tau} \mathrm{L}-\mathrm{TAU} \quad \frac{M \equiv N}{a(M) \equiv a(N)} \mathrm{L}-\mathrm{IN} \quad \frac{M \equiv N}{\bar{a}\langle M\rangle \equiv \bar{a}\langle N\rangle} \mathrm{L}-\mathrm{OUT}
$$

Lemma A.4. [Reduction preserves structural congruence] If $P \equiv Q$ then 
(a) for all $\alpha, P^{\prime}, s, s^{\prime}$, if $s \vdash P \stackrel{\alpha}{\rightarrow} s^{\prime} \vdash P^{\prime}$ then either

$i$. there are $a, M$ such that if $\alpha \equiv \bar{a}\langle M\rangle$ or $\alpha \equiv \tau$, then there are $\beta, Q^{\prime}$ such that $s \vdash Q \stackrel{\beta}{\rightarrow} s^{\prime} \vdash Q^{\prime}, \alpha \equiv \beta$ and $P^{\prime} \equiv Q^{\prime}$, or

ii. there are $a, M$ such that if $\alpha \equiv a(M)$, then for all $\beta$ such that $\alpha \equiv \beta$, there is $Q^{\prime}$ such that $s \vdash Q \stackrel{\beta}{\rightarrow} s^{\prime} \vdash Q^{\prime}$ and $P^{\prime} \equiv Q^{\prime}$, and

(b) for all $\alpha, Q^{\prime}, s, s^{\prime}$, if $s \vdash Q \stackrel{\alpha}{\rightarrow} s^{\prime} \vdash Q^{\prime}$ then either

$i$. there are $a, M$ such that if $\alpha \equiv \bar{a}\langle M\rangle$ or $\alpha \equiv \tau$, then there are $\beta, P^{\prime}$ such that $s \vdash P \stackrel{\beta}{\rightarrow} s \vdash P^{\prime}, \alpha \equiv \beta$ and $P^{\prime} \equiv Q^{\prime}$, or

ii. there are $a, M$ such that if $\alpha \equiv a(M)$, then for all $\beta$ such that $\alpha \equiv \beta$, there is $P^{\prime}$ such that $s \vdash P \stackrel{\beta}{\rightarrow} s^{\prime} \vdash P^{\prime}$ and $P^{\prime} \equiv Q^{\prime}$.

Proof. By induction on the derivations of $P \equiv Q$. 


\section{B Environmental bisimulations of $\mathrm{HO} \pi \mathrm{Pn}$}

\section{Generalities}

Definition B.1. [Contexts]

We define multi-hole contexts for terms $C$ (contexts that have holes for terms) and multi-hole contexts for processes $C_{p}$ (contexts that have holes for processes) as:

$$
\begin{aligned}
D_{p} & :=X \mid{ }^{\prime} C_{p} \\
C_{p} & :=[\cdot]_{i}|0| a(X) \cdot C_{p}\left|\bar{a}\left\langle D_{p}\right\rangle \cdot C_{p}\right|\left(C_{p} \mid C_{p}\right)\left|a\left[C_{p}\right]\right| \nu a \cdot C_{p}\left|! C_{p}\right| \operatorname{run}\left(D_{p}\right) \\
D & ::=[\cdot]_{i}|X|{ }^{\prime} C \\
C & ::=0|a(X) \cdot C| \bar{a}\langle D\rangle . C|(C \mid C)| a[C]|\nu a . C| ! C \mid \operatorname{run}(D)
\end{aligned}
$$

Unless explicitly specified otherwise, the word "context" will denote a context for terms.

Definition B.2. [Context closures] We write

$$
\begin{aligned}
& (\mathcal{E} ; r)^{\circ}=\{(C[\widetilde{M}], C[\widetilde{N}]) \mid b n(C) \cap f n(\widetilde{M}, \widetilde{N})=\emptyset, f n(C) \subseteq r, \quad(\widetilde{M}, \widetilde{N}) \in \mathcal{E}\} \\
& (\mathcal{E} ; r)^{\star}=\{(D[\widetilde{M}], D[\widetilde{N}]) \mid b n(D) \cap f n(\widetilde{M}, \widetilde{N})=\emptyset, f n(D) \subseteq r,(\widetilde{M}, \widetilde{N}) \in \mathcal{E}\}
\end{aligned}
$$

Definition B.3. [Reduction-closed barbed equivalence]

Reduction-closed barbed equivalence $\approx$ is the largest binary relation on configurations indexed with a set of names $r \subseteq s \cap t$ such that when $s \vdash P \approx_{r} t \vdash Q$,

$-s \vdash P \stackrel{\tau}{\rightarrow} s^{\prime} \vdash P^{\prime}$ implies there are $Q^{\prime}$ and $t^{\prime}$ such that $t \vdash Q \Rightarrow t^{\prime} \vdash Q^{\prime}$ and $s^{\prime} \vdash P^{\prime} \approx_{r} t^{\prime} \vdash Q^{\prime}$,

$-s \vdash P \downarrow_{\mu}$ implies $t \vdash Q \Downarrow_{\mu}$ if $\mu$ or $\bar{\mu}$ is in $r$,

- the converse of the above two, on $Q$, and

- for all $R$ such that $f n(R) \cap((s \cup t) \backslash r)=\emptyset$, we have $s \cup f n(R) \vdash P \mid R \approx_{r \cup f n(R)}$ $t \cup f n(R) \vdash Q \mid R$.

Definition B.4. [Non-capturing reduction-closed barbed congruence]

Reduction-closed barbed congruence $\dot{\sim}$ is the largest binary relation on variableclosed configurations indexed with a set of names $r \subseteq s \cap t$ such that when $s \vdash P \dot{\approx}_{r} t \vdash Q$,

$-s \vdash P \stackrel{\tau}{\rightarrow} s^{\prime} \vdash P^{\prime}$ implies there are $Q^{\prime}$ and $t^{\prime}$ such that $t \vdash Q \Rightarrow t^{\prime} \vdash Q^{\prime}$ and $s^{\prime} \vdash P^{\prime} \dot{\approx}_{r} t^{\prime} \vdash Q^{\prime}$,

$-s \vdash P \downarrow_{\mu}$ implies $t \vdash Q \Downarrow_{\mu}$ if $\mu$ or $\bar{\mu}$ is in $r$,

- the converse of the above two, on $Q$, and

- for all $C$ context with holes for processes such that $f n(C) \cap((s \cup t) \backslash r)=$ $b n(C) \cap f n(P, Q)=\emptyset$, we have $s \cup f n(C) \vdash C[P] \dot{\tilde{\sim}}_{r \cup f n(C)} t \cup f n(C) \vdash C[Q]$. 
Note 1. Notice that this definition does not allow capturing names in $r$ (nor $s$ and $t$ ) and is therefore not that of a real congruence.

Definition B.5. [Reduction-closed barbed approximation]

Reduction-closed barbed approximation $\lesssim i s$ the largest binary relation on configurations indexed with a set of names $r \subseteq s \cap t$ such that when $s \vdash P \geqq r t \vee Q$,

$-s \vdash P \stackrel{\tau}{\rightarrow} s^{\prime} \vdash P^{\prime}$ implies there are $Q^{\prime}$ and $t^{\prime}$ such that $t \vdash Q \Rightarrow t^{\prime} \vdash Q^{\prime}$ and $s^{\prime} \vdash P^{\prime} \lesssim r t^{\prime} \vdash Q^{\prime}$,

$-s \vdash P \downarrow_{\mu}$ implies $t \vdash Q \Downarrow_{\mu}$ if $\mu$ or $\bar{\mu}$ is in $r$,

- for all $R$ such that $f n(R) \cap((s \cup t) \backslash r)=\emptyset$, we have $s \cup f n(R) \vdash P \mid R \lesssim r \cup f n(R)$ $t \cup f n(R) \vdash Q \mid R$.

Definition B.6. [Non-capturing reduction-closed barbed pre-congruence]

Reduction-closed barbed pre-congruence $\lesssim$ is the largest binary relation on variableclosed configurations indexed with a set of names $r \subseteq s \cap t$ such that when

$s \vdash P \dot{\lesssim}_{r} t \vdash Q$,

$-s \vdash P \stackrel{\tau}{\rightarrow} s^{\prime} \vdash P^{\prime}$ implies there are $Q^{\prime}$ and $t^{\prime}$ such that $t \vdash Q \Rightarrow t^{\prime} \vdash Q^{\prime}$ and $s^{\prime} \vdash P^{\prime} \dot{\lesssim}_{r} t^{\prime} \vdash Q^{\prime}$,

$-s \vdash P \downarrow_{\mu}$ implies $t \vdash Q \Downarrow_{\mu}$ if $\mu$ or $\bar{\mu}$ is in $r$,

- for all $C$ context with holes for processes such that $f n(C) \cap((s \cup t) \backslash r)=$ $b n(C) \cap f n(P, Q)=\emptyset$, we have $s \cup f n(C) \vdash C[P] \dot{\lesssim}_{r \cup f n(C)} t \cup f n(C) \vdash C[Q]$.

Note 2. Notice that this definition does not allow capturing names in $r$ (nor $s$ and $t$ ) and is therefore not that of a real pre-congruence.

Definition B.7. [Environmental simulation]

$\mathcal{X}$ is an environmental simulation if for all $(s \vdash P) \mathcal{X}_{\mathcal{E} ; r}(t \vdash Q)$,

1. if $s \vdash P \stackrel{\tau}{\rightarrow} s^{\prime} \vdash P^{\prime}$ then there is $t^{\prime} \vdash Q^{\prime}$ such that $t \vdash Q \stackrel{\tau}{\Rightarrow} t^{\prime} \vdash Q^{\prime}$ and $\left(s^{\prime} \vdash P^{\prime}\right) \mathcal{X}_{\mathcal{E} ; r}\left(t^{\prime} \vdash Q^{\prime}\right)$,

2. if $s \vdash P \stackrel{a(M)}{\longrightarrow} s \vdash P^{\prime}$ with $a \in r$, then for all $(M, N) \in(\mathcal{E} ; r)^{\star}$ there is $t^{\prime} \vdash Q^{\prime}$ such that $t \vdash Q \stackrel{a(N)}{\Longrightarrow} t^{\prime} \vdash Q^{\prime}$ and $\left(s \vdash P^{\prime}\right) \mathcal{X}_{\mathcal{E} ; r}\left(t^{\prime} \vdash Q^{\prime}\right)$,

3. if $s \vdash P \stackrel{\bar{a}\langle M\rangle}{\longrightarrow} s \vdash P^{\prime}$ with $a \in r$, then there are $t^{\prime} \vdash Q^{\prime}$ and $N$ such that $t \vdash Q \stackrel{\bar{a}\langle N\rangle}{\Longrightarrow} t^{\prime} \vdash Q^{\prime}$ and $\left(s \vdash P^{\prime}\right) \mathcal{X}_{(M, N) \oplus \mathcal{E} ; r}\left(t^{\prime} \vdash Q^{\prime}\right)$,

4. for all $l \in r$ and $\left({ }^{\prime} P_{1},{ }^{\prime} Q_{1}\right) \in \mathcal{E}$, we have $\left(s \vdash P \mid l\left[P_{1}\right]\right) \mathcal{X}_{\mathcal{E} ; r}\left(t \vdash Q \mid l\left[Q_{1}\right]\right)$, and

5. for all $n \notin s \cup t$, we have $(s, n \vdash P) \mathcal{X}_{\mathcal{E} ; r, n}(t, n \vdash Q)$.

Definition B.8. [Environmental bisimulation] $\mathcal{X}$ is an environmental bisimulation if for all $(s \vdash P) \mathcal{X}_{\mathcal{E} ; r}(t \vdash Q)$,

1. if $s \vdash P \stackrel{\tau}{\rightarrow} s^{\prime} \vdash P^{\prime}$ then there is $t^{\prime} \vdash Q^{\prime}$ such that $t \vdash Q \stackrel{\tau}{\Rightarrow} t^{\prime} \vdash Q^{\prime}$ and $\left(s^{\prime} \vdash P^{\prime}\right) \mathcal{X}_{\mathcal{E} ; r}\left(t^{\prime} \vdash Q^{\prime}\right)$, 
2. if $s \vdash P \stackrel{a(M)}{\longrightarrow} s \vdash P^{\prime}$ with $a \in r$, then for all $(M, N) \in(\mathcal{E} ; r)^{\star}$ there is $t^{\prime} \vdash Q^{\prime}$ such that $t \vdash Q \stackrel{a(N)}{\Longrightarrow} t^{\prime} \vdash Q^{\prime}$ and $\left(s \vdash P^{\prime}\right) \mathcal{X}_{\mathcal{E} ; r}\left(t^{\prime} \vdash Q^{\prime}\right)$,

3. if $s \vdash P \stackrel{\bar{a}\langle M\rangle}{\longrightarrow} s \vdash P^{\prime}$ with $a \in r$, then there are $t^{\prime} \vdash Q^{\prime}$ and $N$ such that $t \vdash Q \stackrel{\bar{a}\langle N\rangle}{\Longrightarrow} t^{\prime} \vdash Q^{\prime}$ and $\left(s \vdash P^{\prime}\right) \mathcal{X}_{(M, N) \oplus \mathcal{E} ; r}\left(t^{\prime} \vdash Q^{\prime}\right)$,

4. for all $l \in r$ and $\left({ }^{\prime} P_{1},{ }^{\prime} Q_{1}\right) \in \mathcal{E}$, we have $\left(s \vdash P \mid l\left[P_{1}\right]\right) \mathcal{X}_{\mathcal{E} ; r}\left(t \vdash Q \mid l\left[Q_{1}\right]\right)$,

5. for all $n \notin s \cup t$, we have $(s, n \vdash P) \mathcal{X}_{\mathcal{E} ; r, n}(t, n \vdash Q)$, and

6 . the converse of the three first clauses, on $Q$ 's transitions.

Definition B.9. [Context closure of an environmental relation] We define

$$
\begin{aligned}
\mathcal{X}^{\star}=\{(\mathcal{E}, r, s, P, t, Q) \mid & P \equiv P_{0} \mid P_{1}, \\
& Q \equiv Q_{0} \mid Q_{1}, \\
& \left(s, r^{\prime} \vdash P_{0}\right) \mathcal{X}_{\mathcal{E}^{\prime} ; r, r^{\prime}}\left(t, r^{\prime} \vdash Q_{0}\right), \\
& \left(P_{1}, Q_{1}\right) \in\left(\mathcal{E}^{\prime} ; r r^{\prime}\right)^{\circ}, \\
& \mathcal{E} \subseteq\left(\mathcal{E}^{\prime} ; r r^{\prime}\right)^{\star}, \\
& \left.r^{\prime} \cap(s \cup t)=\emptyset\right\}
\end{aligned}
$$

Definition B.10. [Environmental simulation up-to context]

$\mathcal{X}$ is an environmental simulation up-to context if for all $(s \vdash P) \mathcal{X}_{\mathcal{E} ; r}(t \vdash Q)$,

1. if $s \vdash P \stackrel{\tau}{\rightarrow} s^{\prime} \vdash P^{\prime}$ then there is $t^{\prime} \vdash Q^{\prime}$ such that $t \vdash Q \stackrel{\tau}{\Rightarrow} t^{\prime} \vdash Q^{\prime}$ and $\left(s^{\prime} \vdash P^{\prime}\right) \mathcal{X}_{\mathcal{E} ; r}^{\star}\left(t^{\prime} \vdash Q^{\prime}\right)$,

2. if $s \vdash P \stackrel{a(M)}{\longrightarrow} s \vdash P^{\prime}$ with $a \in r$, then for all $(M, N) \in(\mathcal{E} ; r)^{\star}$ there is $t^{\prime} \vdash Q^{\prime}$ such that $t \vdash Q \stackrel{a(N)}{\Longrightarrow} t^{\prime} \vdash Q^{\prime}$ and $\left(s \vdash P^{\prime}\right) \mathcal{X}_{\mathcal{E} ; r}^{\star}\left(t^{\prime} \vdash Q^{\prime}\right)$,

3. if $s \vdash P \stackrel{\bar{a}\langle M\rangle}{\longrightarrow} s \vdash P^{\prime}$ with $a \in r$, then there are $t^{\prime} \vdash Q^{\prime}$ and $N$ such that $t \vdash Q \stackrel{\bar{a}\langle N\rangle}{\Longrightarrow} t^{\prime} \vdash Q^{\prime}$ and $\left(s \vdash P^{\prime}\right) \mathcal{X}_{(M, N) \oplus \mathcal{E} ; r}^{\star}\left(t^{\prime} \vdash Q^{\prime}\right)$,

4. for all $l \in r$ and $\left({ }^{\prime} P_{1},{ }^{\prime} Q_{1}\right) \in \mathcal{E}$, we have $\left(s \vdash P \mid l\left[P_{1}\right]\right) \mathcal{X}_{\mathcal{E} ; r}^{\star}\left(t \vdash Q \mid l\left[Q_{1}\right]\right)$, and

5. for all $n \notin s \cup t$, we have $(s, n \vdash P) \mathcal{X}_{\mathcal{E} ; r, n}(t, n \vdash Q)$.

Definition B.11. [Environmental bisimulation up-to context]

$\mathcal{X}$ is an environmental bisimulation up-to context if for all $(s \vdash P) \mathcal{X}_{\mathcal{E} ; r}(t \vdash Q)$,

1. if $s \vdash P \stackrel{\tau}{\rightarrow} s^{\prime} \vdash P^{\prime}$ then there is $t^{\prime} \vdash Q^{\prime}$ such that $t \vdash Q \stackrel{\tau}{\Rightarrow} t^{\prime} \vdash Q^{\prime}$ and $\left(s^{\prime} \vdash P^{\prime}\right) \mathcal{X}_{\mathcal{E} ; r}^{\star}\left(t^{\prime} \vdash Q^{\prime}\right)$,

2. if $s \vdash P \stackrel{a(M)}{\longrightarrow} s \vdash P^{\prime}$ with $a \in r$, then for all $(M, N) \in(\mathcal{E} ; r)^{\star}$ there is $t^{\prime} \vdash Q^{\prime}$ such that $t \vdash Q \stackrel{a(N)}{\Longrightarrow} t^{\prime} \vdash Q^{\prime}$ and $\left(s \vdash P^{\prime}\right) \mathcal{X}_{\mathcal{E} ; r}^{\star}\left(t^{\prime} \vdash Q^{\prime}\right)$,

3. if $s \vdash P \stackrel{\bar{a}\langle M\rangle}{\longrightarrow} s \vdash P^{\prime}$ with $a \in r$, then there are $t^{\prime} \vdash Q^{\prime}$ and $N$ such that $t \vdash Q \stackrel{\bar{a}\langle N\rangle}{\Longrightarrow} t^{\prime} \vdash Q^{\prime}$ and $\left(s \vdash P^{\prime}\right) \mathcal{X}_{(M, N) \oplus \mathcal{E} ; r}^{\star}\left(t^{\prime} \vdash Q^{\prime}\right)$,

4. for all $l \in r$ and $\left({ }^{\prime} P_{1},{ }^{\prime} Q_{1}\right) \in \mathcal{E}$, we have $\left(s \vdash P \mid l\left[P_{1}\right]\right) \mathcal{X}_{\mathcal{E} ; r}^{\star}\left(t \vdash Q \mid l\left[Q_{1}\right]\right)$,

5. for all $n \notin s \cup t$, we have $(s, n \vdash P) \mathcal{X}_{\mathcal{E} ; r, n}(t, n \vdash Q)$, and

6 . the converse of the three first clauses, on $Q$ 's transitions. 


\section{Soundness of environmental bisimulations}

Lemma B.12. If $(P, Q) \in(\mathcal{E} ; r)^{\circ}$ and $s \vdash P \stackrel{a(M)}{\longrightarrow} s \vdash P^{\prime}$ then for all $N$ there is $Q^{\prime}$ such that $t \vdash Q \stackrel{a(N)}{\longrightarrow} t \vdash Q^{\prime}$ and $\left(P^{\prime}, Q^{\prime}\right) \in((M, N) \oplus \mathcal{E} ; r)^{\circ}$.

Proof. By induction on the transition derivation $s \vdash P \stackrel{a(M)}{\longrightarrow} s \vdash P^{\prime}$. There are five cases to check.

1. Case In: $C=a(X) \cdot C_{1}$

We have that $s \vdash P=s \vdash a(X) \cdot C_{1}[\widetilde{M}] \stackrel{a(M)}{\longrightarrow} s \vdash C_{1}[\widetilde{M}]\{M / X\}$ and that $t \vdash Q=t \vdash a(X) . C_{1}[\widetilde{N}] \stackrel{a(N)}{\longrightarrow} t \vdash C_{1}[\widetilde{N}]\{N / X\}$. We are done since we replace term $X$ by terms $M$ and $N$, hence $\left(C_{1}[\widetilde{M}]\{M / X\}, C_{1}[\widetilde{N}]\{N / X\}\right) \in$ $((M, N) \oplus \mathcal{E} ; r)^{\circ}$.

2. Case PAR-L: $C=C_{1} \mid C_{2}$

We have that $s \vdash P=s \vdash C_{1}[\widetilde{M}]\left|C_{2}[\widetilde{M}] \stackrel{a(M)}{\longrightarrow} P_{1}^{\prime}\right| C_{2}[\widetilde{M}]$, i.e. $s \vdash$ $C_{1}[\widetilde{M}] \stackrel{a(M)}{\longrightarrow} s \vdash P_{1}^{\prime}$. By the induction hypothesis $t \vdash C_{1}[\widetilde{N}] \stackrel{a(N)}{\longrightarrow} t \vdash Q_{1}^{\prime}$ and $\left(P_{1}^{\prime}, Q_{1}^{\prime}\right) \in((M, N) \oplus \mathcal{E} ; r)^{\circ}$, from which we derive $\left(\left(P_{1}^{\prime} \mid C_{2}[\widetilde{M}]\right),\left(Q_{1}^{\prime} \mid\right.\right.$ $\left.\left.C_{2}[\tilde{N}]\right)\right) \in((M, N) \oplus \mathcal{E} ; r)^{\circ}$ as well as $t \vdash C_{1}[\tilde{N}]\left|C_{2}[\tilde{N}] \stackrel{a(N)}{\longrightarrow} t \vdash Q_{1}^{\prime}\right| C_{2}[\tilde{N}]$.

3. Case PAR-R: $C=C_{1} \mid C_{2}$

Similar.

4. Case Transp: $C=l\left[C_{1}\right]$

We have that $s \vdash P=s \vdash l\left[C_{1}[\widetilde{M}]\right] \stackrel{a(M)}{\longrightarrow} s \vdash l\left[P_{1}^{\prime}\right]$, that is $s \vdash C_{1}[\widetilde{M}] \stackrel{a(M)}{\longrightarrow}$ $s \vdash P_{1}^{\prime}$. By the induction hypothesis, we have that $t \vdash C_{1}[\tilde{N}] \stackrel{a(N)}{\longrightarrow} t \vdash$ $Q_{1}^{\prime}$ and $\left(P_{1}^{\prime}, Q_{1}^{\prime}\right) \in((M, N) \oplus \mathcal{E} ; r)^{\circ}$, from which we derive $\left(l\left[P_{1}^{\prime}\right], l\left[Q_{1}^{\prime}\right]\right) \in$ $((M, N) \oplus \mathcal{E} ; r)^{\circ}$ as well as $t \vdash l\left[C_{1}[\widetilde{N}]\right] \stackrel{a(N)}{\longrightarrow} t \vdash l\left[Q_{1}^{\prime}\right]$.

5. Case Rep: $C=! C_{1}$

We have that $s \vdash P=s \vdash ! C_{1}[\widetilde{M}] \stackrel{a(M)}{\longrightarrow} s \vdash P^{\prime}$, i.e. $s \vdash ! C_{1}[\widetilde{M}] \mid C_{1}[\widetilde{M}] \stackrel{a(M)}{\longrightarrow}$ $s \vdash P^{\prime}$. By the induction hypothesis, we have that $t \vdash ! C_{1}[\tilde{N}] \mid C_{1}[\tilde{N}] \stackrel{a(N)}{\longrightarrow} t \vdash$ $Q^{\prime}$ and $\left(P^{\prime}, Q^{\prime}\right) \in((M, N) \oplus \mathcal{E} ; r)^{\circ}$. Thus $t \vdash ! C_{1}[\tilde{N}] \stackrel{a(N)}{\longrightarrow} t \vdash Q^{\prime}$ and still $\left(P^{\prime}, Q^{\prime}\right) \in((M, N) \oplus \mathcal{E} ; r)^{\circ}$.

Lemma B.13. If $(P, Q) \in(\mathcal{E} ; r)^{\circ}$ and $s \vdash P \stackrel{\bar{a}\langle M\rangle}{\longrightarrow} s \vdash P^{\prime}$ then there are $Q^{\prime}$ and $N$ such that $t \vdash Q \stackrel{\bar{a}\langle N\rangle}{\longrightarrow} t \vdash Q^{\prime},\left(P^{\prime}, Q^{\prime}\right) \in(\mathcal{E} ; r)^{\circ}$ and $(M, N) \in(\mathcal{E} ; r)^{\star}$.

Proof. By induction on the transition derivation $P \stackrel{\bar{a}\langle M\rangle}{\longrightarrow} P^{\prime}$. There are six cases to check.

1. Case Output: $C=\bar{a}\left\langle{ }^{\prime} C_{1}\right\rangle \cdot C_{2}$

We have that $s \vdash P=\bar{a}\left\langle\left\langle^{\prime} C_{1}[\widetilde{M}]\right\rangle \cdot C_{2}[\widetilde{M}] \stackrel{\bar{a}\left\langle{ }^{\prime} C_{1}[\widetilde{M}]\right\rangle}{\longrightarrow} s \vdash C_{2}[\widetilde{M}]\right.$ and that $t \vdash Q_{1}=\bar{a}\left\langle{ }^{\prime} C_{1}[\widetilde{N}]\right\rangle \cdot C_{2}[\widetilde{N}] \stackrel{\bar{a}\left\langle{ }^{\prime} C_{1}[\tilde{N}]\right\rangle}{\longrightarrow} t \vdash C_{2}[\widetilde{N}]$. It is immediate to confirm that $\left({ }^{\prime} C_{1}[\widetilde{M}],{ }^{\prime} C_{1}[\widetilde{N}]\right) \in(\mathcal{E} ; r)^{\star}$ and $\left(C_{2}[\widetilde{M}], C_{2}[\widetilde{N}]\right) \in(\mathcal{E} ; r)^{\circ}$ hold. 
2. Case PAR-L: $C=C_{1} \mid C_{2}$

We have that $s \vdash P=C_{1}[\widetilde{M}]\left|C_{2}[\widetilde{M}] \stackrel{\bar{a}\langle M\rangle}{\longrightarrow} s \vdash P_{1}^{\prime}\right| C_{2}[\widetilde{M}]$, i.e. $s \vdash C_{1}[\widetilde{M}] \stackrel{\bar{a}\langle M\rangle}{\longrightarrow}$ $s \vdash P_{1}^{\prime}$. By the induction hypothesis, we have that $t \vdash C_{1}[\widetilde{N}] \stackrel{\bar{a}\langle N\rangle}{\longrightarrow} t \vdash Q_{1}^{\prime}$ and $\left(P_{1}^{\prime}, Q_{1}^{\prime}\right) \in(\mathcal{E} ; r)^{\circ}$ and $(M, N) \in(\mathcal{E} ; r)^{\star}$. Hence $t \vdash C_{1}[\tilde{N}] \mid C_{2}[\tilde{N}] \stackrel{\bar{a}\langle N\rangle}{\longrightarrow} t \vdash$ $Q_{1}^{\prime} \mid C_{2}[\widetilde{N}]$, and $\left(\left(P_{1}^{\prime} \mid C_{2}[\widetilde{M}]\right),\left(Q_{1}^{\prime} \mid C_{2}[\widetilde{N}]\right)\right) \in(\mathcal{E} ; r)^{\circ}$.

3. Case PAR-R: $C=C_{1} \mid C_{2}$

Similar.

4. Case Transp: $C=l\left[C_{1}\right]$

We have that $s \vdash P=l\left[C_{1}[\widetilde{M}]\right] \stackrel{\bar{a}\langle M\rangle}{\longrightarrow} s \vdash l\left[P_{1}^{\prime}\right]$, i.e. $s \vdash C_{1}[\widetilde{M}] \stackrel{\bar{a}\langle M\rangle}{\longrightarrow} s \vdash P_{1}^{\prime}$. By the induction hypothesis, we have $t \vdash C_{1}[\widetilde{N}] \stackrel{a\langle N\rangle}{\longrightarrow} t \vdash Q_{1}^{\prime},\left(P_{1}^{\prime}, Q_{1}^{\prime}\right) \in(\mathcal{E} ; r)^{\circ}$ and $(M, N) \in(\mathcal{E} ; r)^{\star}$. From this we derive $t \vdash l\left[C_{1}[\tilde{N}]\right] \stackrel{\bar{a}\langle N\rangle}{\longrightarrow} l\left[Q_{1}^{\prime}\right]$ and $\left(l\left[P_{1}^{\prime}\right], l\left[Q_{1}^{\prime}\right]\right) \in(\mathcal{E} ; r)^{\circ}$ and we are done.

5. Case Passiv: $C=l\left[C_{1}\right]$

We have that $s \vdash P=s \vdash l\left[C_{1}[\widetilde{M}]\right] \stackrel{\bar{l}\left\langle{ }^{‘} C_{1}[\widetilde{M}]\right\rangle}{\longrightarrow} 0$. Immediately, we have $t \vdash Q=$ $t \vdash l\left[C_{1}[\widetilde{N}]\right] \stackrel{\bar{l}\left\langle{ }^{\prime} C_{1}[\widetilde{N}]\right\rangle}{\longrightarrow} 0$ with $\left({ }^{\circ} C_{1}[\widetilde{M}],{ }^{\prime} C_{1}[\widetilde{N}]\right) \in(\mathcal{E} ; r)^{\star}$ and $(0,0) \in(\mathcal{E} ; r)^{\circ}$.

6. Case REP: $C=! C_{1}$

We have that $s \vdash P=s \vdash ! C_{1}[\widetilde{M}] \stackrel{\bar{a}\langle M\rangle}{\longrightarrow} s \vdash P^{\prime}$, i.e. $s \vdash ! C_{1}[\widetilde{M}] \mid C_{1}[\widetilde{M}] \stackrel{\bar{a}\langle M\rangle}{\longrightarrow}$ $s \vdash P^{\prime}$. By the induction hypothesis, we have $t \vdash ! C_{1}[\tilde{N}] \mid C_{1}[\widetilde{N}] \stackrel{a\langle N\rangle}{\longrightarrow} t \vdash Q^{\prime}$, $(M, N) \in(\mathcal{E} ; r)^{\star}$ and $\left(P^{\prime}, Q^{\prime}\right) \in(\mathcal{E} ; r)^{\circ}$, hence $! C_{1}[\widetilde{N}] \stackrel{\bar{a}\langle N\rangle}{\longrightarrow} Q^{\prime}$ and we are done.

Proposition B.14. [Non-interference of names]

1. If $s \vdash P_{0}\left|P_{1} \stackrel{\alpha}{\rightarrow} s^{\prime} \vdash P_{0}^{\prime}\right| P_{1}$ then for any $x$, we can assume that $s, x \vdash$ $P_{0}\left|P_{1} \stackrel{\alpha}{\rightarrow} s^{\prime}, x \vdash P_{0}^{\prime}\right| P_{1}$ using implicit $\alpha$-conversion in $s \vdash P_{0} \mid P_{1}$.

2. If $s \vdash P_{0} \stackrel{\alpha}{\Rightarrow} s^{\prime} \vdash P_{0}^{\prime}$ then for any $P_{1}$ such that $f n\left(P_{1}\right) \cap\left(s^{\prime} \backslash s\right)$, we have $s \vdash P_{0}\left|P_{1} \stackrel{\alpha}{\Rightarrow} s^{\prime} \vdash P_{0}^{\prime}\right| P_{1}$.

Lemma B.15. [Input and output preserve environmental bisimulation up-to context]

Let $\mathcal{Y}$ be an environmental bisimulation up-to context and $\mathcal{X}=\{(\mathcal{E}, r, s, P, t, Q) \mid$ $\left.(s \vdash P) \mathcal{Y}_{\mathcal{E} ; r}^{\star}(t \vdash Q)\right\}$. Then, for all $(s \vdash P) \mathcal{X}_{\mathcal{E} ; r}(t \vdash Q)$,

1. if $s \vdash P \stackrel{a(M)}{\longrightarrow} s \vdash P^{\prime}$ with a in $r$, then for all $(M, N) \in(\mathcal{E} ; r)^{\star}$ there are $Q^{\prime}$, $t^{\prime}$ such that $t \vdash Q \stackrel{a(N)}{\Longrightarrow} t^{\prime} \vdash Q^{\prime}$ and $\left(s \vdash P^{\prime}\right) \mathcal{X}_{\mathcal{E} ; r}\left(t^{\prime} \vdash Q^{\prime}\right)$,

2. if $s \vdash P \stackrel{\bar{a}\langle M\rangle}{\longrightarrow} s \vdash P^{\prime}$ with a in $r$, then there are $Q^{\prime}, N$, $t^{\prime}$ such that $t \vdash$ $Q \stackrel{\bar{a}\langle N\rangle}{\Longrightarrow} t^{\prime} \vdash Q^{\prime}$ and $\left(s \vdash P^{\prime}\right) \mathcal{X}_{(M, N) \oplus \mathcal{E} ; r}\left(t^{\prime} \vdash Q^{\prime}\right)$, and

3. the converse of the above two hold for $Q$ 's transitions too.

Proof. Suppose $(s \vdash P) \mathcal{Y}_{\mathcal{E} ; r}^{\star}(t \vdash Q)$, therefore for some $P_{0}, P_{1}, Q_{0}, Q_{1}, \mathcal{E}^{\prime}, r^{\prime}$, we have $P \equiv P_{0}\left|P_{1}, Q \equiv Q_{0}\right| Q_{1}, r^{\prime} \cap(s \cup t)=\emptyset, \mathcal{E} \subseteq\left(\mathcal{E}^{\prime} ; r r^{\prime}\right)^{\star},\left(s, r^{\prime} \vdash\right.$ 
$\left.P_{0}\right) \mathcal{Y}_{\mathcal{E}^{\prime} ; r r^{\prime}}\left(t, r^{\prime} \vdash Q_{0}\right)$ and $\left(P_{1}, Q_{1}\right) \in\left(\mathcal{E}^{\prime} ; r r^{\prime}\right)^{\circ}$. We are going to analyse all the possible input/output transitions.

1. Case: Input

There are two subcases for this transition:

(a) Subcase: $s \vdash P_{0}\left|P_{1} \stackrel{a(M)}{\longrightarrow} s \vdash P_{0}^{\prime}\right| P_{1}$

By $s \vdash P_{0}\left|P_{1} \stackrel{a(M)}{\longrightarrow} s \vdash P_{0}^{\prime}\right| P_{1}$, we know that we have $s \vdash P_{0} \stackrel{a(M)}{\longrightarrow} s \vdash P_{0}^{\prime}$, and therefore $s, r^{\prime} \vdash P_{0} \stackrel{a(M)}{\longrightarrow} s, r^{\prime} \vdash P_{0}^{\prime}$. By $\mathcal{E} \subseteq\left(\mathcal{E}^{\prime} ; r r^{\prime}\right)^{\star}$, we have $(\mathcal{E} ; r)^{\star} \subseteq\left(\mathcal{E}^{\prime} ; r r^{\prime}\right)^{\star}$, and thus, by $\left(s, r^{\prime} \vdash P_{0}\right) \mathcal{Y}_{\mathcal{E}^{\prime} ; r r^{\prime}}\left(t, r^{\prime} \vdash Q_{0}\right)$ and $s, r^{\prime} \vdash P_{0} \stackrel{a(M)}{\longrightarrow} s, r^{\prime} \vdash P_{0}^{\prime}$, we have (i) $t, r^{\prime} \vdash Q_{0} \stackrel{a(N)}{\longrightarrow} t^{\prime}, r^{\prime} \vdash Q_{0}^{\prime}$ and (ii) $\left(s, r^{\prime} \vdash P_{0}^{\prime}\right) \mathcal{Y}_{\mathcal{E}^{\prime} ; r r^{\prime}}^{\star}\left(t^{\prime}, r^{\prime} \vdash Q_{0}^{\prime}\right)$. (i) tells us that $t \vdash Q_{0} \mid Q_{1} \stackrel{a(N)}{\Longrightarrow}$ $t^{\prime} \vdash Q_{0}^{\prime} \mid Q_{1} \equiv Q^{\prime}$, and (ii) tells us, using the up-to techniques, that $\left(s \vdash P^{\prime}\right) \mathcal{Y}_{\mathcal{E} ; r}^{\star}\left(t^{\prime} \vdash Q^{\prime}\right)$, hence $\left(s \vdash P^{\prime}\right) \mathcal{X}_{\mathcal{E} ; r}\left(t^{\prime} \vdash Q^{\prime}\right)$.

(b) Subcase: $s \vdash P_{0}\left|P_{1} \stackrel{a(M)}{\longrightarrow} P_{0}\right| P_{1}^{\prime}$

By $s \vdash P_{0}\left|P_{1} \stackrel{a(M)}{\longrightarrow} s \vdash P_{0}\right| P_{1}^{\prime}$, we know that we have $s \vdash P_{1} \stackrel{a(M)}{\longrightarrow}$ $s \vdash P_{1}^{\prime}$, and therefore that we have $s, r^{\prime} \vdash P_{1} \stackrel{a(M)}{\longrightarrow} s, r^{\prime} \vdash P_{1}^{\prime}$. By Lemma B.12, we have that (i) $t, r^{\prime} \vdash Q_{1} \stackrel{a(N)}{\longrightarrow} t, r^{\prime} \vdash Q_{1}^{\prime}$ and (ii) $\left(P_{1}^{\prime}, Q_{1}^{\prime}\right) \in$ $\left((M, N) \oplus \mathcal{E}^{\prime} ; r r^{\prime}\right)^{\circ}$. Since $(M, N) \in(\mathcal{E} ; r)^{\star} \subseteq\left(\mathcal{E}^{\prime} ; r r^{\prime}\right)^{\star}$, (ii) actually implies $\left(P_{1}^{\prime}, Q_{1}^{\prime}\right) \in\left(\mathcal{E}^{\prime} ; r r^{\prime}\right)^{\circ}$, and thus we have, for $Q^{\prime} \equiv Q_{0} \mid Q_{1}^{\prime},(s \vdash$ $\left.P^{\prime}\right) \mathcal{Y}_{\mathcal{E} ; r}^{\star}\left(t \vdash Q^{\prime}\right)$, that is, $\left(s \vdash P^{\prime}\right) \mathcal{X}_{\mathcal{E} ; r}\left(t \vdash Q^{\prime}\right)$. (i) implies $t \vdash Q_{0} \mid$ $Q_{1} \stackrel{a(N)}{\longrightarrow} t \vdash Q_{0} \mid Q_{1}^{\prime}$, and we are done.

2. Case: Output

There are two cases for this transition:

(a) Subcase: $s \vdash P_{0}\left|P_{1} \stackrel{\bar{a}\langle M\rangle}{\longrightarrow} s \vdash P_{0}^{\prime}\right| P_{1}$

By $s \vdash P_{0}\left|P_{1} \stackrel{\bar{a}\langle M\rangle}{\longrightarrow} s \vdash P_{0}^{\prime}\right| P_{1}$, we know that we have $s \vdash P_{0} \stackrel{\bar{a}\langle M\rangle}{\longrightarrow} s \vdash P_{0}^{\prime}$, and therefore $s, r^{\prime} \vdash P_{0} \stackrel{\bar{a}\langle M\rangle}{\longrightarrow} s, r^{\prime} \vdash P_{0}^{\prime}$. By $\left(s, r^{\prime} \vdash P_{0}\right) \mathcal{Y}_{\mathcal{E}^{\prime} ; r r^{\prime}}\left(t, r^{\prime} \vdash Q_{0}\right)$ and $s, r^{\prime} \vdash P_{0} \stackrel{\bar{a}\langle M\rangle}{\longrightarrow} s, r^{\prime} \vdash P_{0}^{\prime}$, we have (i) $t, r^{\prime} \vdash Q_{0} \stackrel{\bar{a}\langle N\rangle}{\longrightarrow} t^{\prime}, r^{\prime} \vdash Q_{0}^{\prime}$ and (ii) $\left(s, r^{\prime} \vdash P_{0}^{\prime}\right) \mathcal{Y}_{(M, N) \oplus \mathcal{E}^{\prime} ; r r^{\prime}}^{\star}\left(t^{\prime}, r^{\prime} \vdash Q_{0}^{\prime}\right)$. (i) tells us that $t \vdash Q_{0} \mid Q_{1} \stackrel{\bar{a}\langle N\rangle}{\Longrightarrow}$ $t^{\prime} \vdash Q_{0}^{\prime} \mid Q_{1} \equiv Q^{\prime}$, and (ii) tells us, using the up-to techniques and the fact that $(M, N) \oplus \mathcal{E} \subseteq\left((M, N) \oplus \mathcal{E}^{\prime} ; r r^{\prime}\right)^{\star}$, that $\left(s \vdash P^{\prime}\right) \mathcal{Y}_{(M, N) \oplus \mathcal{E} ; r}^{\star}\left(t^{\prime} \vdash Q^{\prime}\right)$, hence $\left(s \vdash P^{\prime}\right) \mathcal{X}_{(M, N) \oplus \mathcal{E} ; r}\left(t^{\prime} \vdash Q^{\prime}\right)$.

(b) Subcase: $s \vdash P_{0}\left|P_{1} \stackrel{\bar{a}\langle M\rangle}{\longrightarrow} P_{0}\right| P_{1}^{\prime}$

By $s \vdash P_{0}\left|P_{1} \stackrel{\bar{a}\langle M\rangle}{\longrightarrow} s \vdash P_{0}\right| P_{1}^{\prime}$, we know that we have $s \vdash P_{1} \stackrel{\bar{a}\langle M\rangle}{\longrightarrow} s \vdash P_{1}^{\prime}$, and therefore that we have $s, r^{\prime} \vdash P_{1} \stackrel{\bar{a}\langle M\rangle}{\longrightarrow} s, r^{\prime} \vdash P_{1}^{\prime}$. By Lemma B.12, we have that (i) $t, r^{\prime} \vdash Q_{1} \stackrel{\bar{a}\langle N\rangle}{\longrightarrow} t, r^{\prime} \vdash Q_{1}^{\prime}$ and (ii) $\left(P_{1}^{\prime}, Q_{1}^{\prime}\right) \in\left(\mathcal{E}^{\prime} ; r r^{\prime}\right)^{\circ}$ and $(M, N) \in\left(\mathcal{E}^{\prime} ; r r^{\prime}\right)^{\star}$. (ii) means that $\left(s \vdash P^{\prime}\right) \mathcal{Y}_{(M, N) \oplus \mathcal{E} ; r}^{\star}\left(t \vdash Q^{\prime}\right)$ for $Q^{\prime}=Q_{0} \mid Q_{1}^{\prime}$, that is, $\left(s \vdash P^{\prime}\right) \mathcal{X}_{(M, N) \oplus \mathcal{E} ; r}\left(t \vdash Q^{\prime}\right)$, and (i) implies $t \vdash Q_{0}\left|Q_{1} \stackrel{\bar{a}\langle N\rangle}{\longrightarrow} t \vdash Q_{0}\right| Q_{1}^{\prime}$, and we are done. 
3. Case: The converse of the above two cases on $Q$ 's transitions.

Similar to clauses 1 and 2 .

Definition B.16. For all processes $A, B$, we write $A<B$ and $B>A$ if there are $C_{p}$ and $R$ such that $A=C_{p}[R]$ and $B=C_{p}\left[\right.$ run $\left.^{6} R\right]$. We write $P_{0} \leq P_{n}$ if $P_{0}<\cdots<P_{n}$ for some $n \geq 0$, and $A \leq_{n} B$ if $A=P_{0}<\cdots<P_{m}=B$ for some $m \leq n$. We naturally write $A \geq B$ whenever $B \leq A$, and extend $\leq$ and $\geq$ 's definitions to terms and labels.

We use the metavariables $P^{+}$and $P^{-}$along with $P$ when we mean that $P \leq$ $P^{+}$and that $P^{-} \leq P$. (The notations $(\cdot)^{+}$and $(\cdot)^{-}$therefore do not represent operators.) Similarly, we use the metavariables $M^{+}$and $M^{-}$to represent runexpansions and run-erasures of term $M$.

Definition B.17. [run-transition]

We write $s \vdash P \stackrel{\text { run }}{\longrightarrow} s \vdash P^{\prime}$ when $s \vdash P \stackrel{\tau}{\rightarrow} s \vdash P^{\prime}$ is derived using the rule RuN. Then, we write $s \vdash P_{0} \stackrel{\text { run }^{n}}{\longrightarrow} s \vdash P_{n}$ to mean that $s \vdash P_{0} \stackrel{\text { run }}{\longrightarrow} \cdots \stackrel{\text { run }}{\longrightarrow} s \vdash P_{n}$, and $s \vdash P \stackrel{\text { run }}{\Longrightarrow} \vdash Q$ when $s \vdash P \stackrel{\text { run }^{n}}{\longrightarrow} \vdash Q$ for some $n \geq 0$.

Lemma B.18. Let $\mathcal{X}_{S}=\{(\mathcal{E}, P, Q) \mid(P, Q) \in S, \mathcal{E} \subseteq S\}$. If $(\mathcal{E}, P, Q) \in \mathcal{X}_{<}$, then for any $r, s, t$

- if $s \vdash P \stackrel{\text { run }}{\longrightarrow} s \vdash P^{\prime}$ then

- there is $Q^{\prime}$ such that $t \vdash Q \stackrel{\text { run }}{\longrightarrow} t \vdash Q^{\prime}$ and $\left(\mathcal{E}, P^{\prime}, Q^{\prime}\right) \in \mathcal{X}_{<}$, or

- $t \vdash Q \stackrel{\text { run }}{\longrightarrow} t \vdash P \stackrel{\text { run }}{\longrightarrow} t \vdash P^{\prime}$ and $\left(\mathcal{E}, P^{\prime}, P^{\prime}\right) \in \mathcal{X}_{\leq_{1}}$ with $P^{\prime}=Q^{\prime}$,

- if $s \vdash P \stackrel{\tau}{\rightarrow} s, x \vdash P^{\prime}$ (not with the RUN rule) then there is $Q^{\prime}$ such that $t \vdash Q \stackrel{\text { run }}{\longrightarrow} \stackrel{\tau}{\rightarrow} t, x \vdash Q^{\prime}$, and $\left(\mathcal{E}, P^{\prime}, Q^{\prime}\right) \in \mathcal{X}_{\leq}$,

- if $s \vdash P \stackrel{\bar{a}\langle M\rangle}{\longrightarrow} s \vdash P^{\prime}$ then there are $Q^{\prime}, M \leq_{1} N$ such that $t \vdash Q \stackrel{\text { run }}{\longrightarrow} \stackrel{\bar{a}\langle N\rangle}{\longrightarrow} t \vdash$ $Q^{\prime}$, and $\left((M, N) \oplus \mathcal{E}, P^{\prime}, Q^{\prime}\right) \in \mathcal{X}_{\leq}$,

- if $s \vdash P \stackrel{a(M)}{\longrightarrow} s \vdash P^{\prime}$ then for all $(M, N) \in(\mathcal{E} ; r)^{\star}$ there is $Q^{\prime}$ such that $t \vdash Q \stackrel{\text { run }}{\longrightarrow} \stackrel{a(N)}{\longrightarrow} t \vdash Q^{\prime}$, and $\left(\mathcal{E}, P^{\prime}, Q^{\prime}\right) \in \mathcal{X}_{\leq}$,

- the converse on $Q$ 's transitions (without run pre-steps).

Similarly for $>$.

Proof. By induction on the derivation transition of $s \vdash P \stackrel{\alpha}{\rightarrow} s^{\prime} \vdash P^{\prime}$ (or $t \vdash$ $\left.Q \stackrel{\beta}{\rightarrow} t^{\prime} \vdash Q\right)$.

- Output

- Case P's output

There are two subcases: the context outputs, or some $R_{i}$.

* Subcase $s \vdash P=C_{p}[R] \stackrel{\bar{a}\langle M\rangle}{\longrightarrow} s \vdash P^{\prime}$ by an output by the context.

We have $s \vdash Q=C_{p}\left[\operatorname{run}^{\natural} R\right] \stackrel{\bar{a}\langle N\rangle}{\longrightarrow} s \vdash Q^{\prime}$. We are done as either $P^{\prime}=C_{p}^{\prime}[R]<C_{p}^{\prime}\left[\operatorname{run}^{\prime} R\right]=Q^{\prime}$ and $M=N$, or $P^{\prime}=Q^{\prime}$ and $M<N$, hence $\left((M, N) \oplus \mathcal{E}, P^{\prime}, Q^{\prime}\right) \in \mathcal{X}_{\leq}$. 
* Subcase $s \vdash P=C_{p}\left[R_{i}\right] \stackrel{\bar{a}\langle M\rangle}{\longrightarrow} s \vdash C_{p}\left[R_{i}^{\prime}\right]=P^{\prime}$.

We have $s \vdash Q=C_{p}\left[\right.$ run $\left.^{\natural} R_{i}\right] \stackrel{\text { run }}{\longrightarrow} s \vdash C_{p}\left[R_{i}\right] \stackrel{\bar{a}\langle N\rangle}{\longrightarrow} s \vdash C_{p}\left[R_{i}^{\prime}\right]$. We have that $M=N$ and that $P^{\prime}=Q^{\prime}$ hence $\left((M, N) \oplus \mathcal{E}, P^{\prime}, Q^{\prime}\right) \in \mathcal{X}_{\leq}$.

- Case $Q$ 's output:

There is only one subcase, as $R$ cannot output for it is guarded.

* Subcase $s \vdash Q=C_{p}\left[\operatorname{run}^{\natural} R\right] \stackrel{\bar{a}\langle N\rangle}{\longrightarrow} s \vdash P^{\prime}$ by an output from the context.

Then $s \vdash P=s \vdash C_{p}\left[{ }^{[} R\right] \stackrel{\bar{a}\langle M\rangle}{\longrightarrow} s \vdash Q^{\prime}$. We are done as either $P^{\prime}=Q^{\prime}$ - Reduction and $M<N$ or $P^{\prime}<Q^{\prime}$ and $M=n$, hence $\left((M, N) \oplus \mathcal{E}, P^{\prime}, Q^{\prime}\right) \in \mathcal{X}_{\leq}$.

- Case P's reduction:

There are four subcases: the context reduces, sends $R$, receives from $R$, or $R$ reduces.

* Subcase $s \vdash P=C_{p}[R] \stackrel{\tau}{\rightarrow} s^{\prime} \vdash P^{\prime}$ by a reduction by the context. We can do a case analysis on how the transition is done.

1. Subsubcase run-transition.

We have in fact $s \vdash P=C_{p}[R] \stackrel{\text { run }}{\longrightarrow} s \vdash C_{p}^{\prime}[R]$ and also $s \vdash Q=$ $C_{p}\left[\right.$ run $\left.^{6} R\right] \stackrel{\text { run }}{\longrightarrow} s \vdash C_{p}^{\prime}\left[\right.$ run $\left.^{6} R\right]$. Therefore, $\left(\mathcal{E}, P^{\prime}, Q^{\prime}\right) \in \mathcal{X}_{<} \subseteq \mathcal{X}_{\leq 1}$.

2. Subsubcase alloc-transition.

We have in fact $s \vdash P=C_{p}[R] \stackrel{\tau}{\rightarrow} s^{\prime} \vdash C_{p}^{\prime}[R]$ and also $s \vdash Q=$ $C_{p}\left[\right.$ run $\left.^{\natural} R\right] \stackrel{\tau}{\rightarrow} s^{\prime} \vdash C_{p}^{\prime}\left[\right.$ run $\left.^{\natural} R\right]$. Therefore, $\left(\mathcal{E}, P^{\prime}, Q^{\prime}\right) \in \mathcal{X}_{<} \subseteq \mathcal{X}_{\leq}$.

3. Subsubcase other $\tau$-transition.

We have in fact $s \vdash P=C_{p}[R] \stackrel{\tau}{\rightarrow} s \vdash C_{p}^{\prime}\left[\widetilde{R}^{\prime}\right]$ because it may send $R$ and duplicate or discard it, or substitute some variable in it for an other process. Therefore, we have $s \vdash Q=C_{p}\left[\operatorname{run}^{\prime} R\right] \stackrel{\tau}{\rightarrow} s \vdash$ $C_{p}^{\prime}\left[\widetilde{\text { run }^{6} R^{\prime}}\right]$ with the same number $n$ of copies since the same reaction can be done. We then have $P^{\prime}<\cdots<Q^{\prime}$ with $n$ " $<$ ", hence $\left(\mathcal{E}, P^{\prime}, Q^{\prime}\right) \in \mathcal{X}_{\leq}$.

* Subcase $s \vdash P=C_{p}[R] \stackrel{\tau}{\rightarrow} s \vdash C_{p}^{\prime}\left[R^{\prime}\{M / X\}\right]$ by a communication between the context and $R$.

Then $s \vdash Q=C_{p}\left[\right.$ run $\left.^{6} R\right] \stackrel{\text { run }}{\longrightarrow} s \vdash C_{p}[R] \stackrel{\tau}{\rightarrow} s \vdash C_{p}^{\prime}\left[R^{\prime}\{N / X\}\right]$. Since $M=N$ has to hold, we have $C_{p}^{\prime}\left[R^{\prime}\{M / X\}\right]=C_{p}^{\prime}\left[R^{\prime}\{N / X\}\right]$ hence $\left(\mathcal{E}, P^{\prime}, Q^{\prime}\right) \in \mathcal{X}_{<}$.

* Subcase $s \vdash P=C_{p}[R] \stackrel{\tau}{\rightarrow} s \vdash C_{p}^{\prime}\left[R^{\prime}, A\right]$ by a communication between the context and $R$.

Then $s \vdash Q=C_{p}\left[\right.$ run $\left.^{\natural} R\right] \stackrel{\text { run }}{\longrightarrow} s \vdash C_{p}[R] \stackrel{\tau}{\rightarrow} s \vdash C_{p}^{\prime}\left[R^{\prime}, A\right]$. We have $C_{p}^{\prime}\left[R^{\prime}, A\right]=C_{p}^{\prime}\left[R^{\prime}, A\right]$, hence $\left(\mathcal{E}, P^{\prime}, Q^{\prime}\right) \in \mathcal{X}_{\leq}$.

* Subcase $s \vdash P=C_{p}[R] \stackrel{\tau}{\rightarrow} s^{\prime} \vdash C_{p}\left[R^{\prime}\right]$ by a reduction of some $R$.

Then $s \vdash Q=C_{p}\left[\right.$ run $\left.^{\prime} R\right] \stackrel{\text { run }}{\longrightarrow} s \vdash C_{p}[R] \stackrel{\tau}{\rightarrow} s^{\prime} \vdash C_{p}\left[R^{\prime}\right]$. We have $C_{p}\left[R^{\prime}\right]=C_{p}\left[R^{\prime}\right]$ hence $\left(\mathcal{E}, P^{\prime}, Q^{\prime}\right) \in \mathcal{X}_{\leq}$.

- Case $Q$ 's reduction:

There are only two subcases, either the context reduces, or some run around $R$ is consumed. All the other subcases would imply $R$, but it is not in a redex position. 
* Subcase $s \vdash Q \stackrel{\tau}{\rightarrow} s^{\prime} \vdash Q^{\prime}$ by a reduction from the context. We can do a case analysis on how the transition was done.

1. Subsubcase run-transition.

We have in fact $s \vdash Q=C_{p}\left[\right.$ run $\left.^{\natural} R\right] \stackrel{\text { run }}{\longrightarrow} s \vdash C_{p}^{\prime}\left[\right.$ run $\left.^{\natural} R\right]$. Therefore $s \vdash P=C_{p}[R] \stackrel{\text { run }}{\longrightarrow} s \vdash C_{p}^{\prime}[R]$ and $\left(\mathcal{E}, C_{p}^{\prime}[R], s, C_{p}^{\prime}\left[\right.\right.$ run $\left.\left.^{\prime} R\right]\right) \in \mathcal{X}_{<}$.

2. Subsubcase alloc-transition.

Similarly.

3. Subsubcase other $\tau$-transition.

We have in fact $s \vdash Q=C_{p}\left[\right.$ run $\left.^{\natural} R\right] \stackrel{\tau}{\rightarrow} s \vdash C_{p}^{\prime}\left[\widetilde{\text { run }^{\natural} R^{\prime}}\right]$ since the transition may substitute a variable in $R$ for a process. Therefore we have $s \vdash P=C_{p}\left[R^{\prime}\right] \stackrel{\tau}{\rightarrow} s \vdash C_{p}^{\prime}\left[\widetilde{R}^{\prime}\right]$, and we then have $P^{\prime}<$ $\cdots<Q^{\prime}$, hence $\left(\mathcal{E}, P^{\prime}, Q^{\prime}\right) \in \mathcal{X}_{\leq}$.

* Subcase $s \vdash Q=C_{p}\left[\right.$ run $\left.^{\natural} R\right] \stackrel{\text { run }}{\longrightarrow} s \vdash C_{p}[R]$. We are done since - Input: $Q^{\prime}=P$, hence $\left(\mathcal{E}, P, Q^{\prime}\right) \in \mathcal{X}_{\leq_{1}}$.

- Case $P$ 's input:

There are two subcases: the context inputs, or $R$ does.

* Subcase $s \vdash P=C_{p}[R] \stackrel{a(M)}{\longrightarrow} s \vdash C_{p}^{\prime}\left[R^{\prime}\{M / X\}, \widetilde{M}\right]$ by an input by the context.

Then, for all $(M, N) \in(<; s)^{\star} \subseteq \leq_{1}$, we have $s \vdash Q=C_{p}[$ run $R] \stackrel{a(N)}{\longrightarrow}$ $s \vdash C_{p}^{\prime}\left[\operatorname{run}^{\natural} R^{\prime}\{N / X\}, \widetilde{N}\right]$. We are done as $P^{\prime}=s \vdash C_{p}^{\prime}\left[R, R^{\prime}\{M / X\}, \widetilde{M}\right]<$ $\cdots<C_{p}^{\prime}\left[\operatorname{run}^{6} R^{\prime}\{N / X\}, \widetilde{N}\right]=Q^{\prime}$ hence $\left(\mathcal{E}, P^{\prime}, Q^{\prime}\right) \in \mathcal{X}_{\leq}$.

* Subcase $s \vdash P=C_{p}[R] \stackrel{a(M)}{\longrightarrow} s \vdash C_{p}\left[R^{\prime}\{M / X\}\right]=P^{\prime}$.

Then, for all $(M, N) \in(<; s)^{\star} \subseteq \leq_{1}$, we have $s \vdash Q=C_{p}\left[\right.$ run $\left.^{\natural} R\right] \stackrel{\text { run }}{\longrightarrow}$ $s \vdash C_{p}[R] \stackrel{a(N)}{\longrightarrow} s \vdash C_{p}\left[R^{\prime}\{N / X\}\right]=Q^{\prime}$. Since $M \leq_{1} N$, we have $C_{p}\left[R^{\prime}\{M / X\}\right]<\cdots<C_{p}\left[R^{\prime}\{N / X\}\right]$ hence $\left(\mathcal{E}, P^{\prime}, Q^{\prime}\right) \in \mathcal{X}_{\leq}$.

- Case $Q$ 's input:

There is only one subcase, as $R$ cannot input for it is guarded.

$* s \vdash Q=C_{p}\left[\right.$ run $\left.^{\natural} R\right] \stackrel{a(N)}{\longrightarrow} s \vdash C_{p}^{\prime}\left[\right.$ run $\left.^{\natural} R^{\prime}\{N / X\}, N\right]$ by an input from the context, and thus for all $(M, N) \in(<; s)^{\star} \subseteq \leq_{1}$, we have $s \vdash P=s \vdash C_{p}[R] \stackrel{a(M)}{\longrightarrow} s \vdash C_{p}^{\prime}\left[R^{\prime}\{M / X\}, M\right]$. We are done as $C_{p}^{\prime}\left[R^{\prime}\{M / X\}, M\right]<\cdots<C_{p}^{\prime}\left[\operatorname{run}^{\natural} R^{\prime}\{N / X\}, N\right]$, hence $\left(\mathcal{E}, P^{\prime}, Q^{\prime}\right) \in$ $\mathcal{X}_{\leq}$

Corollary B.19. If $P<Q$ and $s \vdash P \stackrel{\text { run }}{\Longrightarrow} s \vdash P^{\prime}$ then $s \vdash Q \stackrel{\text { run }}{\Longrightarrow} s \vdash Q^{\prime}$ and $P^{\prime}=Q^{\prime}$ or $P^{\prime}<Q^{\prime}$, and conversely.

Proof. By induction on the number of run's in $s \vdash P \stackrel{\text { run }^{n}}{\Longrightarrow} s \vdash P^{\prime}$, using Lemma B.18.

Corollary B.20. If $P \leq_{m} Q$ and $s \vdash P \stackrel{\text { run }}{\Longrightarrow} s \vdash P^{\prime}$ then $s \vdash Q \stackrel{\text { run }}{\Longrightarrow} s \vdash Q^{\prime}$ and $P^{\prime} \leq_{n} Q^{\prime}$, with $n \leq m$ and conversely.

Proof. By induction on the number of $<$ 's in $P \leq Q$, using Corollary B.19. 
Lemma B.21. Suppose $P_{0} \leq P_{m}$ and $s \vdash P_{0} \stackrel{\text { run }}{\longrightarrow} \stackrel{\alpha}{\longrightarrow} s^{\prime} \vdash P_{0}^{\prime}$ without using the RuN or REACT- $\{\mathrm{L}, \mathrm{R}\}$ rules for transition $\stackrel{\alpha}{\rightarrow}$. Then, $s \vdash P_{m} \stackrel{\text { run }}{\longrightarrow} \stackrel{\beta}{\rightarrow} s^{\prime} \vdash P_{m}^{\prime}$, $\alpha \leq \beta$ and $P_{0}^{\prime} \leq P_{m}^{\prime}$. And conversely.

Proof. By induction on the number of " $<$ " in $\leq$.

Direct

- Case 0

Trivial.

- Case $\neq 0$

We have $s \vdash P_{0} \stackrel{\text { run }}{\longrightarrow} s \vdash P_{0}^{\prime \prime} \stackrel{\alpha}{\rightarrow} s^{\prime} \vdash P_{0}^{\prime}$ and $P_{0} \leq P_{m}$, so we can apply Corollary B.20 to have $s \vdash P_{m} \stackrel{\text { run }}{\Longrightarrow} s \vdash P_{m}^{\prime \prime}$ and $P_{0}^{\prime \prime} \leq P_{m}^{\prime \prime}$, that is $P_{0}^{\prime \prime}<$ $P_{1}^{\prime \prime}<\cdots<P_{m}^{\prime \prime}$ with at most as many "<". Then, $s \vdash P_{0}^{\prime \prime} \stackrel{\alpha}{\rightarrow} s^{\prime} \vdash P_{0}^{\prime}$, so, by Lemma B.18, we have $s \vdash P_{1}^{\prime \prime} \stackrel{\text { run }}{\longrightarrow} \stackrel{\gamma}{\rightarrow} s^{\prime} \vdash P_{1}^{\prime}$ with $\alpha \leq \gamma$ and $P_{0}^{\prime} \leq P_{1}^{\prime}$. We can apply the induction hypothesis to $s \vdash P_{1}^{\prime \prime} \stackrel{\text { run }}{\longrightarrow} \stackrel{\gamma}{\rightarrow} s^{\prime} \vdash P_{1}^{\prime}$ and $P_{1}^{\prime \prime} \leq P_{m}^{\prime \prime}$ to obtain that $s \vdash P_{m}^{\prime \prime} \stackrel{\text { run }}{\longrightarrow} \stackrel{\beta}{\rightarrow} s^{\prime} \vdash P_{m}^{\prime}, \alpha \leq \beta$ and $P_{1}^{\prime} \leq P_{m}^{\prime}$. Therefore, $P_{0}^{\prime} \leq P_{1}^{\prime} \leq P_{m}^{\prime}, \alpha \leq \gamma \leq \beta$, and $s \vdash P_{m} \stackrel{\text { run }}{\Longrightarrow} s \vdash P_{m}^{\prime \prime} \stackrel{\text { run }}{\longrightarrow} \stackrel{\beta}{\rightarrow} s^{\prime} \vdash P_{m}^{\prime}$ as desired.

- Converse

By Corollary B.20, we can get rid of the initial run's and just consider the simpler hypothesis $P_{0} \leq P_{m}$ and $s \vdash P_{m} \stackrel{\beta}{\rightarrow} s^{\prime} \vdash P_{m}^{\prime}$.

- Case 0

Trivial

- Case $\neq 0$

By Lemma B.18 and $P_{0}<\cdots<P_{m-1}<P_{m}$ we have that $s \vdash P_{m-1} \stackrel{\gamma}{\rightarrow}$ $s^{\prime} \vdash P_{m-1}^{\prime}$ with $P_{m-1}^{\prime} \leq P_{m}^{\prime}$ and $\gamma \leq \beta$. We just call the induction hypothesis on $P_{0} \leq P_{m-1}$ and $s \vdash P_{m-1} \stackrel{\gamma}{\rightarrow} s^{\prime} \vdash P_{m-1}^{\prime}$ and we obtain as desired $s \vdash P_{0} \stackrel{\alpha}{\rightarrow} s^{\prime} \vdash P_{0}^{\prime}$, with $\alpha \leq \gamma \leq \beta$. and $P_{0}^{\prime} \leq P_{m-1}^{\prime} \leq P_{m}^{\prime}$.

Lemma B.22. Suppose $P_{0} \leq P_{m}$ and $s \vdash P_{0} \stackrel{\text { run }}{\longrightarrow} \stackrel{\tau}{\rightarrow} s \vdash P_{0}^{\prime}$ using rule REACT$\{\mathrm{L}, \mathrm{R}\}$ for the $\stackrel{\tau}{\rightarrow}$ transition. Then, $s \vdash P_{m} \stackrel{\text { run }}{\longrightarrow} \stackrel{\tau}{\rightarrow} s \vdash P_{m}^{\prime}$, and $P_{0}^{\prime} \leq P_{m}^{\prime}$. And conversely

Proof. Using Lemma B.21, considering that $P_{0}$ communicates a term $N$.

- We have $s \vdash P_{0} \stackrel{\text { run }}{\longrightarrow} \stackrel{\tau}{\rightarrow} s \vdash P_{0}^{\prime}$, doing a reaction, that is, $s \vdash P_{0} \stackrel{\text { run }}{\longrightarrow} \stackrel{a(N)}{\longrightarrow} s \vdash P_{0 l}$ for some process $N$ and channel $a$. So, by Lemma B.21, we have $s \vdash P_{m} \stackrel{\text { run }}{\Longrightarrow}$ $s \vdash P_{m l} \stackrel{a\left(N^{+}\right)}{\longrightarrow}$. with $P_{0} \leq P_{m l}$. Now, as we also have $s \vdash P_{0} \stackrel{\bar{a}\langle N\rangle}{\longrightarrow} s \vdash P_{0 r}$, by Lemma B.21, we have $s \vdash P_{m l} \stackrel{\text { run }}{\longrightarrow} s \vdash P_{m r} \stackrel{\bar{a}\left\langle N^{+^{\prime}}\right\rangle}{\longrightarrow} \cdot$ with $P_{0} \leq P_{m r}$. As it happens that $P_{m r}$ can reduce through the communication of an expansion of $N$ on channel $a$, we have $s \vdash P_{m r} \stackrel{\tau}{\rightarrow} s \vdash P_{m}^{\prime}$ with $P_{0}^{\prime} \leq P_{m}^{\prime}$ and we are done.

- Converse

Similarly. 
Corollary B.23. The sets $\mathcal{X}_{\leq}$and $\mathcal{X}_{\geq}$are both preserved by input, output and reduction.

Proof. Consequence of Corollary B.20 and Lemmas B.21 and B.22.

Corollary B.24. For any contexts $C$ and its erasures $C_{1}^{-}$and $C_{2}^{-}$, for any processes $\widetilde{P}$ and $\widetilde{Q}$, if $s \vdash C_{1}^{-}[\widetilde{P}] \stackrel{\alpha}{\rightarrow} s, x \vdash C_{1}^{\prime-}[\widetilde{P}]$ then $t \vdash C_{2}^{-}[\widetilde{Q}] \stackrel{\beta}{\Rightarrow} t, x \vdash C_{2}^{\prime-}[\widetilde{Q}]$ with $C_{1}^{\prime-}$ and $C_{2}^{\prime-}$ erasures of $C^{\prime}, \alpha$ and $\beta$ erasures of some $\gamma$, and possibly $x=\emptyset$.

Proof. Consequence of Corollary B.23, more precisely of Lemma B.18 focusing on the cases where the context only does a transition.

Definition B.25. [Minimal transition of run-expanded processes]

Suppose that $A \leq B, s \vdash A \stackrel{\alpha}{\rightarrow} s^{\prime} \vdash A^{\prime}, s \vdash B \stackrel{\text { run }^{n}}{\longrightarrow} \stackrel{\beta}{\rightarrow} s^{\prime} \vdash B^{\prime}$ with $\alpha \leq \beta$ and that $A^{\prime} \leq B^{\prime}$. We say that $s \vdash B \stackrel{\text { run }^{n}}{\longrightarrow} \stackrel{\beta}{\rightarrow} s^{\prime} \vdash B^{\prime}$ is minimal with respect to $s \vdash A \stackrel{\alpha}{\rightarrow} s^{\prime} \vdash A^{\prime}$ if and only if for all $s \vdash B \stackrel{\text { run }^{m}}{\longrightarrow} \stackrel{\gamma}{\rightarrow} s^{\prime} \vdash B^{\prime \prime}$ with $A^{\prime} \leq B^{\prime \prime}$ and $\alpha \leq \gamma$, we have $n \leq m$.

Lemma B.26. [Minimality and run-transition]

Suppose that $s \vdash B \stackrel{\text { run }}{\longrightarrow} s \vdash B^{\prime \prime} \stackrel{\text { run } n^{n-1}}{\longrightarrow} \stackrel{\beta}{\longrightarrow} s^{\prime} \vdash B^{\prime}$ with $n>0$ is minimal with respect to $s \vdash A \stackrel{\alpha}{\rightarrow} s^{\prime} \vdash A^{\prime}$. We have that $s \vdash B^{\prime \prime} \stackrel{\text { run }{ }^{n-1}}{\longrightarrow} \stackrel{\beta}{\rightarrow} s^{\prime} \vdash B^{\prime}$ too is minimal with respect to $s \vdash A \stackrel{\alpha}{\rightarrow} s^{\prime} \vdash A^{\prime}$.

Proof. By reductio ad absurdum. Suppose that $s \vdash B^{\prime \prime} \stackrel{\text { run }}{\longrightarrow} \stackrel{\beta}{\longrightarrow} s^{\prime} \vdash B^{\prime}$ is not minimal with respect to $s \vdash A \stackrel{\alpha}{\rightarrow} s^{\prime} \vdash A^{\prime}$. There must be a minimal transition $s \vdash B^{\prime \prime} \stackrel{\text { run }^{m}}{\longrightarrow} \stackrel{\gamma}{\rightarrow} s^{\prime} \vdash B^{\prime \prime \prime}$ with $s \vdash A^{\prime} \leq s \vdash B^{\prime \prime \prime}, \alpha \leq \gamma$, and $m<n-1$. Then we have a derivation $s \vdash B \stackrel{\text { run }}{\longrightarrow} s \vdash B^{\prime \prime} \stackrel{\text { run }^{m}}{\longrightarrow} \stackrel{\gamma}{\rightarrow} s^{\prime} \vdash B^{\prime \prime \prime}$ of length $m+1<n$ with $s \vdash A^{\prime} \leq s \vdash B^{\prime \prime \prime}$, which contradicts the assumption that $s \vdash B \stackrel{\text { run }}{\longrightarrow} s \vdash$ $B^{\prime \prime} \stackrel{\text { run } n^{n-1}}{\longrightarrow} \stackrel{\beta}{\rightarrow} s^{\prime} \vdash B^{\prime}$ is minimal.

Lemma B.27. [Minimality and contexts]

For all $s \vdash Q \stackrel{\text { run }^{n}}{\longrightarrow} \stackrel{\beta}{\rightarrow} s^{\prime} \vdash Q^{\prime}$ minimal with respect to $s \vdash P \stackrel{\alpha}{\rightarrow} s^{\prime} \vdash P^{\prime}$,

- for all evaluation context $C$ and its erasure $C^{-}, s \vdash C[Q] \stackrel{\text { run }}{\longrightarrow} \stackrel{\beta}{\rightarrow} s^{\prime} \vdash C\left[Q^{\prime}\right]$ is minimal with respect to $s \vdash C^{-}[P] \stackrel{\alpha}{\rightarrow} s^{\prime} \vdash C^{-}\left[P^{\prime}\right]$,

- if $Q=Q_{0}\left|Q_{1}, Q^{\prime}=Q_{0}^{\prime}\right| Q_{1}^{\prime}$, and $P=P_{0} \mid P_{1}$ with $P_{0} \leq Q_{0}, P_{1} \leq Q_{1}$, then for all $l$ and $m, s \vdash l\left[Q_{0}\right]\left|m\left[Q_{1}\right] \stackrel{\text { run }^{n}}{\longrightarrow} \stackrel{\beta}{\rightarrow} s^{\prime} \vdash l\left[Q_{0}^{\prime}\right]\right| m\left[Q_{1}^{\prime}\right]$ is minimal with respect to $s \vdash l\left[P_{0}\right]\left|m\left[P_{1}\right] \stackrel{\alpha}{\rightarrow} s^{\prime} \vdash l\left[P_{0}^{\prime}\right]\right| m\left[P_{1}^{\prime}\right]$.

Proof. Immediate, as none of the above operations can reduce the number of run's that have to be deleted, and as they all preserve membership to $\leq$. 
Definition B.28. [run-erased context closure]

We define the run-erased context closure $(\mathcal{E} ; r)^{-}$of environment $\mathcal{E}$ with names $r$ as $\leq(\mathcal{E} ; r)^{\star} \geq$, that is $\left\{(M, N) \mid M \leq A, N \leq B,(A, B) \in(\mathcal{E} ; r)^{\star}\right\}$. Notice that $(\mathcal{E} ; r)^{-}$may erase run's inside elements related by $\mathcal{E}$ too.

We also write $(s \vdash P) \mathcal{Y}_{\mathcal{E} ; r}^{-}(t \vdash Q)$ if $(s \vdash P \leq) \mathcal{Y}_{\leq \mathcal{E} \geq ; r}^{\star}(\geq t \vdash Q)$ (which implies $\left.\mathcal{Y}^{\star} \subseteq \mathcal{Y}^{-}\right)$. In other words $(s \vdash P) \mathcal{Y}_{\mathcal{E} ; r}^{-}(t \vdash Q)$ if $P \equiv P_{0}\left|P_{1}, Q \equiv Q_{0}\right| Q_{1}$, $\left(s, r^{\prime} \vdash P_{0} \leq\right) \mathcal{Y}_{\mathcal{E}^{\prime} ; r r^{\prime}}\left(\geq t, r^{\prime} \vdash Q_{0}\right),\left({ }^{\prime} P_{1},{ }^{\prime} Q_{1}\right) \in\left(\mathcal{E}^{\prime} ; r r^{\prime}\right)^{-}, \mathcal{E} \subseteq\left(\mathcal{E}^{\prime} ; r r^{\prime}\right)^{-}$, and $r^{\prime} \cap(s \cup t)=\emptyset$.

Corollary B.29. [run-erasure preserves run-erased context closure of environmental bisimulation up-to context]

If $(s \vdash P) \mathcal{Y}_{\mathcal{E}_{;} ;}^{-}(t \vdash Q), P^{-} \leq P, Q^{-} \leq Q$ and $\mathcal{E}^{-} \leq \mathcal{E}$ then $\left(s \vdash P^{-}\right) \mathcal{Y}_{\mathcal{E}^{-} ; r}^{-}(t \vdash$ $\left.Q^{-}\right)$.

Proof. From transitivity of $\leq$ and $\geq$ given by Definition B.16.

Lemma B.30. [Addition of fresh names preserves environmental bisimulation up-to context and its run-erased context closure]

Let $\mathcal{Y}$ be an environmental bisimulation up-to context. If $(s \vdash P) \mathcal{Y}_{\mathcal{E} ; r}^{\star}(t \vdash Q)$ and $l \notin s \cup t$, then $(s, l \vdash P) \mathcal{Y}_{\mathcal{E} ; l \oplus r}^{\star}(t, l \vdash Q)$. Similarly, if $(s \vdash P) \mathcal{Y}_{\mathcal{E} ; r}^{-}(t \vdash Q)$ and $l \notin s \cup t$, then $(s, l \vdash P) \mathcal{Y}_{\mathcal{E} ; l \oplus r}^{-}(t, l \vdash Q)$.

Proof. By simple set arithmetic and use of definitions.

- Case $\mathcal{Y}^{\star}$

Given $P=P_{0}\left|P_{1}, Q=Q_{0}\right| Q_{1}$ such that $\left(s, x \vdash P_{0}\right) \mathcal{Y}_{\mathcal{E}^{\prime}: r}\left(t, x \vdash Q_{0}\right)$, $\left(P_{1}, Q_{1}\right) \in\left(\mathcal{E}^{\prime} ; r x\right)^{\circ}, x \cap(s \cup t)=\emptyset$, and $\mathcal{E} \subseteq\left(\mathcal{E}^{\prime} ; r x\right)^{\star}$, and assuming $l \neq x$ (otherwise it is immediate), it holds that

- $\left(s, x \vdash P_{0}\right) \mathcal{Y}_{\mathcal{E}^{\prime} ; l \oplus r x}\left(t, x \vdash Q_{0}\right)$ by clause 5 of environmental bisimulation up-to context,

- $\left(P_{1}, Q_{1}\right) \in\left(\mathcal{E}^{\prime} ; l \oplus r\right)^{\circ} \subseteq\left(\mathcal{E}^{\prime} ; l \oplus r x\right)^{\circ}$,

- $\mathcal{E} \subseteq\left(\mathcal{E}^{\prime} ; r x\right)^{\star} \subseteq\left(\mathcal{E}^{\prime} ; l \oplus r x\right)^{\circ}$,

- $x \notin s \cup t$

Therefore, $(s \vdash P) \mathcal{Y}_{\mathcal{E} ; l \oplus r}^{\star}(t \vdash Q)$ holds.

- Case $\mathcal{Y}^{-}$

We have some $P^{+} \geq P, Q^{+} \geq Q, \mathcal{E}^{+} \geq \mathcal{E}$ such that $\left(s \vdash P^{+}\right) \mathcal{Y}_{\mathcal{E}^{+} ; r}^{\star}\left(t \vdash Q^{+}\right)$.

Therefore, according to the above case, we have $\left(s \vdash P^{+}\right) \mathcal{Y}_{\mathcal{E}^{+} ; l \oplus r}^{\star}\left(t \vdash Q^{+}\right)$, hence $(s \vdash P) \mathcal{Y}_{\mathcal{\mathcal { E }} ; l \oplus r}^{-}(t \vdash Q)$ by Definition B.28.

Lemma B.31. [Spawning preserves context closure of environmental bisimulation up-to context]

Let $\mathcal{Y}$ be an environmental bisimulation up-to context. For all $(s \vdash P) \mathcal{Y}_{\mathcal{E} ; r}^{\star}(t \vdash$ $Q), l \in r$ and $\left({ }^{\prime} P_{2},{ }^{\prime} Q_{2}\right) \in \mathcal{E}$, we have $\left(s \vdash P \mid l\left[P_{2}\right]\right) \mathcal{Y}_{\mathcal{E} ; r}^{\star}\left(t \vdash Q \mid l\left[Q_{2}\right]\right)$.

Proof. We have $P \equiv P_{0} \mid P_{1}$ and $Q \equiv Q_{0} \mid Q_{1}$, with $\left(s, r^{\prime} \vdash P_{0}\right) \mathcal{Y}_{\mathcal{E}^{\prime} ; r r^{\prime}}\left(t, r^{\prime} \vdash Q_{0}\right)$, $\left(P_{1}, Q_{1}\right) \in\left(\mathcal{E}^{\prime} ; r r^{\prime}\right)^{\circ}, \mathcal{E} \subseteq\left(\mathcal{E}^{\prime} ; r r^{\prime}\right)^{\star}$, and $r^{\prime} \notin s \cup t$. By $\left({ }^{\prime} P_{2},{ }^{\prime} Q_{2}\right) \in \mathcal{E}$, we have either (' $\left.P_{2},{ }^{\prime} Q_{2}\right) \in \mathcal{E}^{\prime}$ or $\left(P_{2}, Q_{2}\right) \in\left(\mathcal{E}^{\prime} ; r r^{\prime}\right)^{\circ}$. In the former case, it holds 
that $\left(s, r^{\prime} \vdash P_{0} \mid l\left[P_{2}\right]\right) \mathcal{Y}_{\mathcal{E}^{\prime} ; r r^{\prime}}^{\star}\left(t, r^{\prime} \vdash Q_{0} \mid l\left[Q_{2}\right]\right)$ by clause 4 of environmental bisimulation up-to context, hence $\left(s \vdash P_{0}\left|l\left[P_{2}\right]\right| P_{1}\right) \mathcal{Y}_{\mathcal{E} ; r}^{\star}\left(t \vdash Q_{0}\left|l\left[Q_{2}\right]\right| Q_{1}\right)$ upto environment, context and name creation. In the latter case, we immediately have $\left(P_{1}\left|l\left[P_{2}\right], Q_{1}\right| l\left[Q_{2}\right]\right) \in\left(\mathcal{E}^{\prime} ; r r^{\prime}\right)^{\circ}$, hence $\left(s \vdash P \mid l\left[P_{2}\right]\right) \mathcal{Y}_{\mathcal{E} ; r}^{\star}\left(t \vdash Q \mid l\left[Q_{2}\right]\right)$.

Lemma B.32. [run-transitions of $\left.(\mathcal{E} ; r)^{\circ}\right]$

Suppose that $\left(P_{1}, Q_{1}\right)=(C[\widetilde{M}], C[\widetilde{N}]) \in(\mathcal{E} ; r)^{\circ}$ and that $s \vdash P_{1} \stackrel{\text { run }}{\longrightarrow} s \vdash$ $P_{1}^{\prime}$. Then there is a $Q_{1}^{\prime}$ such that $t \vdash Q_{1} \stackrel{\text { run }}{\longrightarrow} t \vdash Q_{1}^{\prime}$ and either $\left(P_{1}^{\prime}, Q_{1}^{\prime}\right)=$ $\left(C^{\prime}[\widetilde{M}], C^{\prime}[\widetilde{N}]\right) \in(\mathcal{E} ; r)^{\circ}$ or $\left({ }^{\prime} P_{1}^{\prime},{ }^{\prime} Q_{1}^{\prime}\right)=\left({ }^{\prime} C_{p}\left[\operatorname{run}\left(\widetilde{M^{\prime}}\right), A\right],{ }^{\prime} C_{p}\left[\operatorname{run}\left(\widetilde{N}^{\prime}\right), B\right]\right) \in$ $(\mathcal{E} ; r)^{-}$with $(A, B)$ in redex position (i.e. not under a run, a $\nu$, an $a(\cdot)$ or an $\bar{a}\langle\cdot\rangle)$ and $\left(\widetilde{M}^{\prime}, ' A\right)=\widetilde{M},\left(\widetilde{N}^{\prime},{ }^{\prime} B\right)=\widetilde{N}$.

Proof. By induction on the transition derivation of $s \vdash P_{1} \stackrel{\text { run }}{\longrightarrow} s \vdash P 1^{\prime}$. The only case of interest is the RUN one, developed below. The others (PAR-L, PARR,REP and TRANSP) are straightforward.

1. Case Run: $C=\operatorname{run}\left(C_{1}\right)$

There are two subcases

(a) $C_{1}={ }^{\prime} C_{1}$

We have $s \vdash P_{1}=s \vdash \operatorname{run}\left({ }^{\prime} C_{1}[\widetilde{M}]\right) \stackrel{\text { run }}{\longrightarrow} s \vdash C_{1}[\widetilde{M}]$ and $t \vdash Q_{1}=t \vdash$ $\operatorname{run}\left({ }^{\prime} C_{1}[\tilde{N}]\right) \stackrel{\text { run }}{\longrightarrow} t \vdash C_{1}[\tilde{N}]$ with $\left(C_{1}[\widetilde{M}], C_{2}[\tilde{N}]\right) \in(\mathcal{E} ; r)^{\circ}$.

(b) $C_{1}=[\cdot]$

We have $s \vdash P_{1}=\operatorname{run}\left({ }^{\prime} A\right) \stackrel{\text { run }}{\longrightarrow} s \vdash A, t \vdash Q_{1} \stackrel{\text { run }}{\longrightarrow} t \vdash B$ with $\left({ }^{\prime} A,{ }^{\prime} B\right) \in$ $\mathcal{E} \subseteq(\mathcal{E} ; r)^{-}$(we can assume that $(A, B) \notin(\mathcal{E} ; r)^{\circ}$, otherwise we could have handled this situation in the above subcase) and obviously $(A, B)$ in redex position.

Lemma B.33. [Create-transitions of $(\mathcal{E} ; r)^{\circ}$ ]

Suppose that $\left(P_{1}, Q_{1}\right)=(C[\widetilde{M}], C[\widetilde{N}]) \in(\mathcal{E} ; r)^{\circ}$ and that $s \vdash P_{1} \stackrel{\tau}{\rightarrow} s, a \vdash P_{1}^{\prime}$ by the CREATE rule. Then there is a $Q_{1}^{\prime}$ such that $t \vdash Q_{1} \stackrel{\tau}{\rightarrow} t, a \vdash Q_{1}^{\prime}$ and $\left(P_{1}^{\prime}, Q_{1}^{\prime}\right)=\left(C^{\prime}[\widetilde{M}], C^{\prime}[\widetilde{N}]\right) \in(\mathcal{E} ; r a)^{\circ}$.

Proof. By induction on the transition derivation of $s \vdash P_{1} \stackrel{\tau}{\rightarrow} s, a \vdash P_{1}^{\prime}$. The only case of interest is the CREATE one, developed below. The others (PAR-L, PAR-R, REP and TRANSP) are straightforward.

1. Case CReate: $C=\nu a . C_{1}$

We have $s \vdash P_{1}=s \vdash \nu a . C_{1}[\widetilde{M}] \stackrel{\tau}{\rightarrow} s, a \vdash C_{1}[\widetilde{M}]$ and $t \vdash Q_{1}=t \vdash \nu a . C_{1}[\widetilde{N}]^{\tau} \rightarrow$ $t, a \vdash C_{1}[\widetilde{N}]$ with $\left(C_{1}[\widetilde{M}], C_{1}[\widetilde{N}]\right) \in(\mathcal{E} ; r a)^{\circ}$.

Lemma B.34. [Non-run non-alloc $\tau$-transitions of $\left.(\mathcal{E} ; r)^{\circ}\right]$

Suppose that $\left(P_{1}, Q_{1}\right) \in(\mathcal{E} ; r)^{\circ}$ and that $s \vdash P_{1} \stackrel{\tau}{\rightarrow} s \vdash P_{1}^{\prime}$ is not derived with Run nor CREATE. Then there is a $Q_{1}^{\prime}$ such that $t \vdash Q_{1} \stackrel{\tau}{\rightarrow} t \vdash Q_{1}^{\prime}$ and $\left(P_{1}^{\prime}, Q_{1}^{\prime}\right)=$ $(\mathcal{E} ; r)^{\circ}$.

Proof. By induction on the transition derivation of $s \vdash P_{1} \stackrel{\tau}{\rightarrow} s \vdash P 1^{\prime}$. One case of interest is the REACT-L one, developed below. The others (REACT-R, PAR-L, PAR-R, REP and TRANSP) are similar or straightforward. 
- Case React-L: $C=C 1 \mid C 2$

We have $s \vdash C_{1}[\widetilde{M}]\left|C_{2}[\widetilde{M}] \stackrel{\tau}{\rightarrow} s \vdash P_{1}^{\prime}\right| P_{2}^{\prime}$ with $s \vdash C_{1}[\widetilde{M}] \stackrel{\bar{a}\langle M\rangle}{\longrightarrow} s \vdash P_{1}^{\prime}$ and $s \vdash C_{2}[\widetilde{M}] \stackrel{a(M)}{\longrightarrow} s \vdash P_{2}^{\prime}$ So, by Lemmas B.12 and B.13 we have $t \vdash$ $C_{1}[\widetilde{N}] \stackrel{\bar{a}\langle N\rangle}{\longrightarrow} t \vdash Q_{1}^{\prime}$ with $\left(P_{1}^{\prime}, Q_{1}^{\prime}\right) \in(\mathcal{E} ; r)^{\circ}$ and $(M, N) \in(\mathcal{E} ; r)^{\star}$, and $t \vdash$ $C_{2}[\tilde{N}] \stackrel{a(N)}{\longrightarrow} t \vdash Q_{2}^{\prime}$ with $\left(P_{2}^{\prime}, Q_{2}^{\prime}\right) \in((M, N) \oplus \mathcal{E} ; r)^{\circ}=(\mathcal{E} ; r)^{\circ}$. Therefore, $t \vdash C_{1}[\widetilde{N}]\left|C_{2}[\widetilde{N}] \stackrel{\tau}{\rightarrow} t \vdash Q_{1}^{\prime}\right| Q_{2}^{\prime}$ and $\left(P_{1}^{\prime}\left|P_{2}^{\prime}, Q_{1}^{\prime}\right| Q_{2}^{\prime}\right) \in(\mathcal{E} ; r)^{\circ}$.

Lemma B.35. [Reduction and environmental bisimulation up-to context]

Let $\mathcal{Y}$ be an environmental bisimulation up-to context. If $(s \vdash P) \mathcal{Y}_{\mathcal{E} ; r}^{\star}(t \vdash Q)$ and $s \vdash P \rightarrow s^{\prime} \vdash P^{\prime}$ then there is a $Q^{\prime}$ such that $t \vdash Q \Rightarrow t^{\prime} \vdash Q^{\prime}$ and $\left(s^{\prime} \vdash\right.$ $\left.P^{\prime}\right) \mathcal{Y}_{\mathcal{E} ; r}^{-}\left(t^{\prime} \vdash Q^{\prime}\right)$.

Proof. Suppose $(s \vdash P) \mathcal{Y}_{\mathcal{E} ; r}^{\star}(t \vdash Q)$, therefore for some $P_{0}, P_{1}, Q_{0}, Q_{1}, \mathcal{E}^{\prime}, r^{\prime}$, we have $P \equiv P_{0}\left|P_{1}, Q \equiv Q_{0}\right| Q_{1}, r^{\prime} \cap(s \cup t)=\emptyset, \mathcal{E} \subseteq\left(\mathcal{E}^{\prime} ; r r^{\prime}\right)^{\star},\left(s, r^{\prime} \vdash\right.$ $\left.P_{0}\right) \mathcal{Y}_{\mathcal{E}^{\prime} ; r r^{\prime}}\left(t, r^{\prime} \vdash Q_{0}\right)$ and $\left(P_{1}, Q_{1}\right) \in\left(\mathcal{E}^{\prime} ; r r^{\prime}\right)^{\circ}$.

We are going to analyse all the possible reduction transitions. We recall that $\mathcal{Y}^{\star} \subseteq \mathcal{Y}^{-}$.

1. Case: $s \vdash P \stackrel{\tau}{\rightarrow} s^{\prime} \vdash P^{\prime}$. We have four cases for the transitions of $P_{0} \mid P_{1}$ :

(a) Subcase $s, r^{\prime} \vdash P_{0} \stackrel{\tau}{\rightarrow} s^{\prime}, r^{\prime} \vdash P_{0}^{\prime}$

By $\left(s, r^{\prime} \vdash P_{0}\right) \mathcal{Y}_{\mathcal{E}^{\prime} ; r r^{\prime}}\left(t, r^{\prime} \vdash Q_{0}\right)$, we have that $t, r^{\prime} \vdash Q_{0} \Rightarrow t^{\prime}, r^{\prime} \vdash Q_{0}^{\prime}$ and $\left(s^{\prime}, r^{\prime} \vdash P_{0}^{\prime}\right) \mathcal{Y}_{\mathcal{E}^{\prime}: r r^{\prime}}^{\star^{\prime}}\left(t^{\prime}, r^{\prime} \vdash Q_{0}^{\prime}\right)$. Therefore, by $t, r^{\prime} \vdash Q_{0} \mid Q_{1} \Rightarrow t^{\prime}, r^{\prime} \vdash$ $Q_{0}^{\prime} \mid Q_{1}$ we have $t \vdash Q_{0}\left|Q_{1} \Rightarrow t^{\prime} \vdash Q_{0}^{\prime}\right| Q_{1}$ since the created names can be guaranteed not in $f n\left(Q_{1}\right)$, and by up-to context and environment and name creation, we have $\left(s^{\prime} \vdash P_{0}^{\prime} \mid P_{1}\right) \mathcal{Y}_{\mathcal{E} ; r}^{\star}\left(t^{\prime} \vdash Q_{0}^{\prime} \mid Q_{1}\right)$

(b) Subcase $s, r^{\prime} \vdash P_{1} \stackrel{\tau}{\rightarrow} s^{\prime}, r^{\prime} \vdash P_{1}^{\prime}$

There are several cases, depending on the last derivation rule used.

i. Non-run non-alloc transition, $s^{\prime}=s$

By Lemma B.34, we have $t, r^{\prime} \vdash Q_{1} \stackrel{\tau}{\rightarrow} t, r^{\prime} \vdash Q_{1}^{\prime}$ and $\left(P_{1}^{\prime}, Q_{1}^{\prime}\right) \in$ $\left(\mathcal{E}^{\prime} ; r r^{\prime}\right)^{\circ}$. Therefore, $t \vdash Q_{1} \stackrel{\tau}{\rightarrow} t \vdash Q_{1}^{\prime}$, hence $t \vdash Q_{0}\left|Q_{1} \stackrel{\tau}{\rightarrow} t \vdash Q_{0}\right| Q_{1}^{\prime}$. Finally, $\left(s^{\prime} \vdash P^{\prime}\right) \mathcal{Y}_{\mathcal{E} ; r}^{\star}\left(t^{\prime} \vdash Q^{\prime}\right)$ with $t^{\prime}=t$ and we are done.

ii. Create transition, $s^{\prime}=s, a$

By Lemma B.33, we have $t, r^{\prime} \vdash Q_{1} \stackrel{\tau}{\rightarrow} t, r^{\prime}, a \vdash Q_{1}^{\prime}$, hence $t \vdash Q_{0} \mid$ $Q_{1} \stackrel{\tau}{\rightarrow} t, a \vdash Q_{0} \mid Q_{1}^{\prime}$, and $\left(P_{1}^{\prime}, Q_{1}^{\prime}\right) \in\left(\mathcal{E} ; r r^{\prime} a\right)^{\circ}$. By freshness of $a$, we can use clause 5 of environmental bisimulation up-to and have $\left(s, r^{\prime}, a \vdash P_{0}\right) \mathcal{Y}_{\mathcal{E}^{\prime} ; r r^{\prime} a}\left(t, r^{\prime}, a \vdash Q_{0}\right)$ as well as $\left(r^{\prime}, a\right) \cap(s \cup t)=\emptyset$. Finally, $\mathcal{E} \subseteq\left(\mathcal{E}^{\prime} ; r r^{\prime}\right)^{\star} \subseteq\left(\mathcal{E}^{\prime} ; r r^{\prime} a\right)^{\star}$, giving $\left(s^{\prime} \vdash P^{\prime}\right) \mathcal{Y}_{\mathcal{E} ; r}^{\star}\left(t^{\prime} \vdash Q^{\prime}\right)$ and we are done.

iii. run transition, $s^{\prime}=s$

By Lemma B.32, we have $t, r^{\prime} \vdash Q_{1} \stackrel{\text { run }}{\longrightarrow} t, r^{\prime} \vdash Q_{1}^{\prime}$ (hence $t \vdash Q_{0} \mid$ $\left.Q_{1} \stackrel{\text { run }}{\longrightarrow} t \vdash Q_{0} \mid Q_{1}^{\prime}\right)$ and either $\left(P_{1}^{\prime}, Q_{1}^{\prime}\right)=\left(C^{\prime}[\widetilde{M}], C^{\prime}[\widetilde{N}]\right) \in\left(\mathcal{E}^{\prime} ; r r^{\prime}\right)^{\circ}$ or $\left({ }^{\prime} P_{1}^{\prime},{ }^{\prime} Q_{1}^{\prime}\right)=\left({ }^{\prime} C_{p}\left[\operatorname{run}\left(\widetilde{M^{\prime}}\right), A\right],{ }^{\prime} C_{p}\left[\operatorname{run}\left(\widetilde{N}^{\prime}\right), B\right]\right) \in\left(\mathcal{E}^{\prime} ; r r^{\prime}\right)^{-}$with $(' A, ' B) \in \mathcal{E}^{\prime}$. Therefore, $\left(s \vdash P^{\prime}\right) \mathcal{Y}_{\mathcal{E} ; r}^{-}\left(t \vdash Q^{\prime}\right)$ and we are done. 
(c) Subcase $s, r^{\prime} \vdash P_{0} \stackrel{\bar{a}\langle M\rangle}{\longrightarrow} s, r^{\prime} \vdash P_{0}^{\prime} \quad s, r^{\prime} \vdash P_{1} \stackrel{a(M)}{\longrightarrow} s, r^{\prime} \vdash P_{1}^{\prime}$

By $\left(s, r^{\prime} \vdash P_{0}\right) \mathcal{Y}_{\mathcal{E}^{\prime} ; r r^{\prime}}\left(t, r^{\prime} \vdash Q_{0}\right)$ and clause 4 of environmental bisimulation up-to context, we have $t, r^{\prime} \vdash Q_{0} \stackrel{\bar{a}\langle N\rangle}{\Longrightarrow} t^{\prime}, r^{\prime} \vdash Q_{0}^{\prime}$ and also $\left(s, r^{\prime} \vdash P_{0}^{\prime}\right) \mathcal{Y}_{(M, N) \oplus \mathcal{E}^{\prime} ; r r^{\prime}}^{\star}\left(t^{\prime}, r^{\prime} \vdash Q_{0}^{\prime}\right)$. Also, since $s, r^{\prime} \vdash P_{1} \stackrel{a(M)}{\longrightarrow} s, r^{\prime} \vdash P_{1}^{\prime}$ we have by Lemma B.12 that $t, r^{\prime} \vdash Q_{1} \stackrel{a(N)}{\longrightarrow} t, r^{\prime} \vdash Q_{1}^{\prime}$ and $\left(P_{1}^{\prime}, Q_{1}^{\prime}\right) \in$ $\left(\mathcal{E}^{\prime} \cup\{(M, N)\} ; r r^{\prime}\right)^{\circ}$.

Decomposing the transitions we know that, for some possibly empty set $y$ of names, $t, r^{\prime} \vdash Q_{0} \stackrel{\tau}{\Rightarrow} t, r^{\prime}, y \vdash Q_{0}^{\prime \prime} \stackrel{a(N)}{\longrightarrow} t, r^{\prime}, y \vdash Q_{0}^{\prime \prime \prime} \stackrel{\tau}{\Rightarrow} t^{\prime}, r^{\prime} \vdash Q_{0}^{\prime}$. Also, by $t, r^{\prime} \vdash Q_{1} \stackrel{a(N)}{\longrightarrow} t, r^{\prime} \vdash Q_{1}^{\prime}$ we have by $t, r^{\prime}, y \vdash Q_{1} \stackrel{a(N)}{\longrightarrow} t, r^{\prime}, y \vdash Q_{1}^{\prime}$. Thus, we have $t, r^{\prime} \vdash Q_{0}\left|Q_{1} \stackrel{\tau}{\Rightarrow} t, r^{\prime}, y \vdash Q_{0}^{\prime \prime}\right| Q_{1}$ by PAR-L, $t, r^{\prime}, y \vdash Q_{0}^{\prime \prime} \mid Q_{1} \stackrel{\tau}{\rightarrow}$ $t, r^{\prime}, y Q_{0}^{\prime \prime \prime} \mid Q_{1}^{\prime}$ by REACT-R, and finally $t, r^{\prime}, y \vdash Q_{0}^{\prime \prime \prime}\left|Q_{1}^{\prime} \stackrel{\tau}{\Rightarrow} t^{\prime}, r^{\prime} \vdash Q_{0}^{\prime}\right| Q_{1}^{\prime}$ by PAR-L. We can then therefore derive $t \vdash Q_{0}\left|Q_{1} \Rightarrow t^{\prime} \vdash Q_{0}^{\prime}\right| Q_{1}^{\prime}$.

By $\left(P_{1}^{\prime}, Q_{1}^{\prime}\right) \in\left(\mathcal{E}^{\prime} \cup\{(M, N)\} ; r r^{\prime}\right)^{\circ}$, we also easily have $\left(P_{1}^{\prime}, Q_{1}^{\prime}\right) \in$ $\left(\mathcal{E}^{\prime} \cup\{(M, N)\} ; r r^{\prime}\right)^{\circ}$, and we can derive up-to context from $\left(s, r^{\prime} \vdash\right.$ $\left.P_{0}^{\prime}\right) \mathcal{Y}_{(M, N) \oplus \mathcal{E}^{\prime} ; r r^{\prime}}^{\star}\left(t^{\prime}, r^{\prime} \vdash Q_{0}^{\prime}\right)$ that $\left(s \vdash P_{0}^{\prime} \mid P_{1}^{\prime}\right) \mathcal{Y}_{\mathcal{E} ; r}^{\star}\left(t^{\prime} \vdash Q_{0}^{\prime} \mid Q_{1}^{\prime}\right)$.

(d) Subcase $s \vdash P_{0} \stackrel{a(M)}{\longrightarrow} s \vdash P_{0}^{\prime} \quad s \vdash P_{1} \stackrel{\bar{a}\langle M\rangle}{\longrightarrow} s \vdash P_{1}^{\prime}$

By $s \vdash P_{1} \stackrel{\bar{a}\langle M\rangle}{\longrightarrow} s \vdash P_{1}^{\prime}$ we have $s, r^{\prime} \vdash P_{1} \stackrel{\bar{a}\langle M\rangle}{\longrightarrow} s, r^{\prime} \vdash P_{1}^{\prime}$, and then by Lemma B.13, we have that $t, r^{\prime} \vdash Q_{1} \stackrel{\bar{a}\langle N\rangle}{\longrightarrow} t, r^{\prime} \vdash Q_{1}^{\prime}$ and $(M, N) \in$ $\left(\mathcal{E}^{\prime} ; r r^{\prime}\right)^{\star}$ as well as $\left(P_{1}^{\prime}, Q_{1}^{\prime}\right) \in\left(\mathcal{E}^{\prime} ; r r^{\prime}\right)^{\circ}$. By clause 2 of environmental bisimulation up-to context and the input of $P_{0}$, we have $t, r^{\prime} \vdash Q_{0} \stackrel{a(N)}{\Longrightarrow}$ $t^{\prime}, r^{\prime} \vdash Q_{0}^{\prime}$ and $\left(P_{0}^{\prime}\right) \mathcal{Y}_{\mathcal{E}^{\prime} ; r r^{\prime}}^{\star}\left(Q_{0}^{\prime}\right)$.

Again, we can compose the transitions and obtain $t, r^{\prime} \vdash Q_{0} \mid Q_{1} \stackrel{\tau}{\Rightarrow} t^{\prime}, r^{\prime} \vdash$ $Q_{0}^{\prime} \mid Q_{1}^{\prime}$ as expected.

By $\left(P_{1}^{\prime}, Q_{1}^{\prime}\right) \in\left(\mathcal{E}^{\prime} ; r r^{\prime}\right)^{\circ}$, we also have $\left(P_{1}^{\prime}, Q_{1}^{\prime}\right) \in\left(\mathcal{E}^{\prime} ; r r^{\prime}\right)^{\circ}$, and we can then derive up-to context from $\left(s, r^{\prime} \vdash P_{0}^{\prime}\right) \mathcal{Y}_{\mathcal{E}^{\prime} ; r r^{\prime}}^{\star}\left(t^{\prime}, r^{\prime} \vdash Q_{0}^{\prime}\right)$ that $(s \vdash$ $\left.P_{0}^{\prime} \mid P_{1}^{\prime}\right) \mathcal{Y}_{\mathcal{E} ; r}^{\star}\left(t^{\prime} \vdash Q_{0}^{\prime} \mid Q_{1}^{\prime}\right)$.

2. Case: $t \vdash Q$ reduces.

Conversely.

Lemma B.36. [run-expanded output with spawning]

Suppose that $\left(s \vdash P_{0} \mid l\left[P_{1}\right]\right) \mathcal{Y}_{\mathcal{E} ; r}^{\star}\left(t \vdash Q_{0} \mid l\left[Q_{1}\right]\right)$ for an environmental bisimulation up-to context $\mathcal{Y}$ with $l \in r$ and that $s \vdash P_{1} \stackrel{\text { run }^{n}}{\longrightarrow} \stackrel{\bar{a}\langle M\rangle}{\longrightarrow} s \vdash P_{1}^{\prime}$ is minimal with respect to $s \vdash P_{1}^{-} \stackrel{\bar{a}\left\langle M^{-}\right\rangle}{\longrightarrow} s \vdash P_{1}^{\prime-}$. Then $t \vdash Q_{0}\left|l\left[Q_{1}\right] \stackrel{\bar{a}\langle N\rangle}{\longrightarrow} t^{\prime} \vdash Q_{0}^{\prime}\right| l\left[Q_{1}^{\prime}\right]$, and $\left(s \vdash P_{0}\right) \mathcal{Y}_{(M, N) \oplus\left({ }^{\prime} P_{1}^{\prime},{ }^{\prime} Q_{1}^{\prime}\right) \oplus \mathcal{E} ; r}^{-}\left(t^{\prime} \vdash Q_{0}^{\prime}\right)$

Proof. By induction on $n$.

- Case $n=0$

Immediate by Lemma B.15 used twice (once for the output of $M$ and $N$, and then once more for the passivation of $P_{1}^{\prime}$ and $\left.Q_{1}^{\prime}\right)$ and by the fact that $\mathcal{Y}^{\star} \subseteq \mathcal{Y}^{-}$. 
- Case $n>0$

By Lemma B.35 and Lemma B.26, we have two possible subcases preserving minimality after the first run-transition of $s \vdash P_{0} \mid l\left[P_{1}\right] \stackrel{\text { runn }}{\Longrightarrow} \stackrel{\bar{a}\langle M\rangle}{\longrightarrow} s \vdash$ $P_{0} \mid l\left[P_{1}^{\prime}\right]$, namely $s \vdash P_{0}\left|l\left[P_{1}\right] \stackrel{\text { run }}{\longrightarrow} s \vdash P_{0}\right| l\left[P_{1}^{\prime \prime}\right]$.

- Subcase "still in $\mathcal{Y}^{\star}$ "

We have $t \vdash Q_{0}\left|l\left[Q_{1}\right] \stackrel{\tau}{\Rightarrow} t^{\prime \prime} \vdash Q_{0}^{\prime \prime}\right| l\left[Q_{1}^{\prime \prime}\right]$ and $\left(s \vdash P_{0} \mid l\left[P_{1}^{\prime \prime}\right]\right) \mathcal{Y}_{\mathcal{E} ; r}^{\star}\left(t^{\prime \prime} \vdash\right.$ $\left.Q_{0}^{\prime \prime} \mid l\left[Q_{1}^{\prime \prime}\right]\right)$. As $s \vdash P_{0}\left|l\left[P_{1}^{\prime \prime}\right] \stackrel{\text { run } n^{n-1}}{\longrightarrow} \stackrel{\bar{a}\langle M\rangle}{\longrightarrow} s \vdash P_{0}\right| l\left[P_{1}^{\prime}\right]$ is still minimal with respect to $s \vdash P_{0}^{-}\left|l\left[P_{1}^{-}\right] \stackrel{\bar{a}\left\langle M^{-}\right\rangle}{\longrightarrow} s \vdash P_{0}^{-}\right| l\left[P_{1}^{-}\right]$, we can apply the induction hypothesis and get the desired results.

- Subcase "in $\mathcal{Y}^{-} \backslash \mathcal{Y}^{\star}$ "

We have $t \vdash Q_{0}\left|l\left[Q_{1}\right] \stackrel{\text { run }}{\longrightarrow} t \vdash Q_{0}\right| l\left[Q_{1}^{\prime \prime}\right]$ and $\left(s \vdash P_{0} \mid l\left[P_{1}^{\prime \prime}\right]\right) \mathcal{Y}_{\mathcal{E} ; r}^{-}(t \vdash$ $\left.Q_{0}^{\prime \prime} \mid l\left[Q_{1}^{\prime \prime}\right]\right)$, with $\left(P_{1}^{\prime \prime}, Q_{1}^{\prime \prime}\right)=\left(C_{p}[\operatorname{run}(\widetilde{M}), A], C_{p}[\operatorname{run}(\widetilde{N}), B]\right)$ with $(A, B)$ in redex position such that $\left({ }^{\circ} A,{ }^{\prime} B\right) \in \mathcal{E}^{\prime}$ and $(A, B) \notin\left(\mathcal{E}^{\prime} ; r r^{\prime}\right)^{\circ}$ for some $\mathcal{E}^{\prime}, r^{\prime}$ such that $\mathcal{E} \subseteq\left(\mathcal{E}^{\prime} ; r r^{\prime}\right)^{\star}, P_{0}\left|l\left[P_{1}\right] \equiv P_{A}\right| P_{B}, Q_{0}\left|l\left[Q_{1}\right] \equiv Q_{A}\right| Q_{B}$, $\left(s, r^{\prime} \vdash P_{A}\right) \mathcal{Y}_{\mathcal{E}^{\prime} ; r r^{\prime}}\left(t, r^{\prime} \vdash Q_{A}\right),\left(P_{B}, Q_{B}\right) \in\left(\mathcal{E}^{\prime} ; r r^{\prime}\right)^{\circ}, r^{\prime} \cap(s \cup t)=\emptyset$.

By $s \vdash P_{0}^{-}\left|l\left[P_{1}^{-}\right] \stackrel{\bar{a}\left\langle M^{-}\right\rangle}{\longrightarrow} s \vdash P_{0}^{-}\right| l\left[P_{1}^{\prime-}\right], P_{1}=C_{p}\left[\right.$ run $(\widetilde{M})$, run $\left.^{\natural} A\right]$, $P_{1}^{\prime \prime}=C_{p}[\operatorname{run}(\widetilde{M}), A]$ and $P_{1}^{-} \leq P_{1}^{\prime}$, we know that there is a runerasure $A^{-} \leq A$ such that $A^{-}$is in redex position in $P_{1}^{-}$and that $s, r^{\prime} \vdash A \stackrel{\bar{a}\langle M\rangle}{\longrightarrow} s, r^{\prime} \vdash A^{\prime}$ is minimal with respect to $s, r^{\prime} \vdash A^{-} \stackrel{\bar{a}\left\langle M^{-}\right\rangle}{\longrightarrow}$ $s, r^{\prime} \vdash A^{\prime-}$. Using Lemma B.30 (to add a fresh name $m$ ), and clause 4 of environmental bisimulation up-to context, as well as derived $s, r^{\prime}, m \vdash$ $A^{-} \stackrel{\bar{a}\left\langle M^{-}\right\rangle}{\longrightarrow} s, r^{\prime}, m \vdash A^{\prime-}$, we can apply the induction hypothesis to $\left(s, r^{\prime}, m \vdash P_{A} \mid m[A]\right) \mathcal{Y}_{\mathcal{E}^{\prime} ; r r^{\prime} m}^{\star}\left(t, r^{\prime}, m \vdash Q_{B} \mid m[B]\right)$. We obtain that $t, r^{\prime}, m \vdash Q_{B}\left|m[B] \stackrel{\bar{a}\langle N\rangle}{\Longrightarrow} t^{\prime}, r^{\prime}, m \vdash Q_{B}^{\prime}\right| m\left[B^{\prime}\right]$ and that also $\left(s, r^{\prime}, m \vdash\right.$ $\left.P_{A}\right) \mathcal{Y}_{(M, N) \oplus\left({ }^{\circ} A^{\prime},{ }^{\prime} B^{\prime}\right) \oplus \mathcal{E}^{\prime} ; r r^{\prime} m}^{-}\left(t^{\prime}, r^{\prime}, m \vdash Q_{B}^{\prime}\right)$. From the former, we can derive that $t^{\prime \prime} \vdash Q_{0}\left|l\left[Q_{1}^{\prime \prime}\right] \stackrel{\bar{a}\langle N\rangle}{\Longrightarrow} t^{\prime} \vdash Q_{0}^{\prime}\right| l\left[Q_{1}^{\prime}\right]$, (with $\left(P_{1}^{\prime}, Q_{1}^{\prime}\right)=\left(C_{p}\left[\operatorname{run}(\tilde{N}), A^{\prime}\right]\right.$, $\left.\left.C_{p}\left[\operatorname{run}(\widetilde{N}), B^{\prime}\right]\right)\right)$ and from the latter that $\left(s, r^{\prime}, m \vdash P_{0}\right) \mathcal{Y}_{(M, N) \oplus\left({ }^{\prime} A^{\prime},{ }^{\prime} B^{\prime}\right) \oplus \mathcal{E}^{\prime} ; r r^{\prime} m}^{-}$ $\left(t^{\prime}, r^{\prime}, m \vdash Q_{0}^{\prime}\right)$ up-to context, $\left(s, r^{\prime}, m \vdash P_{0}\right) \mathcal{Y}_{(M, N) \oplus\left({ }^{(} P_{1}^{\prime},{ }^{\prime} Q_{1}^{\prime}\right) \oplus \mathcal{E} ; r r^{\prime} m}^{-}$ $\left(t^{\prime}, r^{\prime}, m \vdash Q_{0}^{\prime}\right)$ up to environment, and $\left(s \vdash P_{0}\right) \mathcal{Y}_{(M, N) \oplus\left({ }^{\circ} P_{1}^{\prime},{ }^{\prime} Q_{1}^{\prime}\right) \oplus \mathcal{E} ; r}^{-}\left(t^{\prime} \vdash\right.$ $\left.Q_{0}^{\prime}\right)$ up-to name creation.

Corollary B.37. [run-expanded output $]$

Suppose that $\left(s \vdash P_{0} \mid P_{1}\right) \mathcal{Y}_{\mathcal{E}: r}^{\star}\left(t \vdash Q_{0} \mid Q_{1}\right)$ for an environmental bisimulation up-to context $\mathcal{Y}$ with $\left(s, x \vdash P_{0}\right) \mathcal{Y}_{\mathcal{E}^{\prime} ; r x}\left(t, x \vdash Q_{0}\right),\left(P_{1}, Q_{1}\right) \in\left(\mathcal{E}^{\prime} ; r x\right)^{\circ}, x \cap(s \cup$ $t)=\emptyset$, and that $s \vdash P_{0}\left|P_{1} \stackrel{\text { run }^{n}}{\longrightarrow} \stackrel{\bar{a}\langle M\rangle}{\longrightarrow} s \vdash P_{0}^{\prime}\right| P_{1}^{\prime}$ is minimal with respect to $s \vdash P_{0}^{-}\left|P_{1}^{-} \stackrel{\bar{a}\left\langle M^{-}\right\rangle}{\longrightarrow} s \vdash P_{0}^{\prime-}\right| P_{1}^{\prime-}$. Then $t \vdash Q_{0}\left|Q_{1} \stackrel{\bar{a}\langle N\rangle}{\Longrightarrow} t^{\prime} \vdash Q_{0}^{\prime}\right| Q_{1}^{\prime}$, and $\left(s \vdash P_{0}^{\prime} \mid P_{1}^{\prime}\right) \mathcal{Y}_{(M, N) \oplus \mathcal{E} ; r}^{-}\left(t^{\prime} \vdash Q_{0}^{\prime} \mid Q_{1}^{\prime}\right)$.

Proof. By induction on $n$. 
- Case $n=0$

As in the above Lemma B.36, immediate by Lemma B.15 and the fact that $\mathcal{Y}^{\star} \subseteq \mathcal{Y}^{-}$.

- Case $n>0$

By Lemma B.35 and Lemma B.26, we have two possible subcases preserving minimality after the first run-transition of $s \vdash P_{0}\left|P_{1} \stackrel{\text { run }^{n}}{\Longrightarrow} \stackrel{\bar{a}\langle M\rangle}{\longrightarrow} s \vdash P_{0}^{\prime}\right| P_{1}^{\prime}$.

- Subcase "still in $\mathcal{Y}^{\star}$ "

We have $s, r^{\prime} \vdash P_{0} \stackrel{\text { run }}{\longrightarrow} s, r^{\prime} \vdash P_{0}^{\prime \prime}$, hence $t \vdash Q_{0} \stackrel{\tau}{\Rightarrow} t^{\prime \prime} \vdash Q_{0}^{\prime \prime}$ and $(s \vdash$ $\left.P_{0}^{\prime \prime}\right) \mathcal{Y}_{\mathcal{E}^{\prime} ; r r^{\prime}}^{\star}\left(t^{\prime \prime} \vdash Q_{0}^{\prime \prime}\right)$, and also that $s, r^{\prime} \vdash P_{0}^{\prime \prime} \stackrel{r^{r u n^{n-1}}}{\longrightarrow} \stackrel{\bar{a}\langle M\rangle}{\longrightarrow} s \vdash P_{0}^{\prime}$ is minimal with respect to $s \vdash P_{0}^{-} \stackrel{\bar{a}\left\langle M^{-}\right\rangle}{\longrightarrow} s \vdash P_{0}^{\prime-}$. Thus, we can apply the induction hypothesis and get (i) $t^{\prime \prime} \vdash Q_{0}^{\prime \prime} \stackrel{\bar{a}\langle N\rangle}{\Longrightarrow} t^{\prime} \vdash Q_{0}^{\prime}$ as well as (ii) $\left(s, r^{\prime} \vdash P_{0}^{\prime}\right) \mathcal{Y}_{(M, N) \oplus \mathcal{E}^{\prime} ; r r^{\prime}}^{-}\left(t^{\prime}, r^{\prime} \vdash Q_{0}^{\prime}\right)$. Therefore, by (i) we have $t \vdash Q_{0}\left|Q_{1} \stackrel{\bar{a}\langle N\rangle}{\Longrightarrow} t^{\prime} \vdash Q_{0}^{\prime}\right| Q_{1}$. and by (ii) we have $\left(s \vdash P_{0}^{\prime} \mid P_{1}\right) \mathcal{Y}_{(M, N) \oplus \mathcal{E} ; r}^{-}$ $\left(t^{\prime} \vdash Q_{0}^{\prime} \mid Q_{1}\right)$ up-to environment and name creation for using $\mathcal{E}$ and removing $r^{\prime}$, and context for spawning $P_{1}$ and $Q_{1}$.

- Subcase "in $\mathcal{Y}^{-} \backslash \mathcal{Y}^{\star}$ "

We have $s, r^{\prime} \vdash P_{1} \stackrel{\text { run }}{\longrightarrow} s, r^{\prime} \vdash P_{1}^{\prime}$. Using Lemma B.30 to add a fresh name $l$ and the fact that $\left(P_{1}, Q_{1}\right) \in\left(\mathcal{E}^{\prime} ; r r^{\prime}\right)^{\circ}$, we have $\left(s, r^{\prime}, l \vdash P_{0} \mid\right.$ $\left.l\left[P_{1}\right]\right) \mathcal{Y}_{\mathcal{E} ; r}^{\star}\left(t, r^{\prime}, l \vdash Q_{0} \mid l\left[Q_{1}\right]\right)$. As $s, r^{\prime}, l \vdash P_{0} \mid l\left[P_{1}\right] \stackrel{\operatorname{run}^{n}}{\Longrightarrow} \stackrel{\bar{a}\langle M\rangle}{\longrightarrow} s, r^{\prime}, l \vdash$ $P_{0} \mid l\left[P_{1}^{\prime}\right]$ is minimal with respect to $s, r^{\prime}, l \vdash P_{0}^{-} \mid l\left[P_{1}^{-}\right] \stackrel{\bar{a}\left\langle M^{-}\right\rangle}{\longrightarrow} s, r^{\prime}, l \vdash$ $P_{0}^{-} \mid l\left[P_{1}^{\prime-}\right]$, we can use Lemma B.36 and have $t, r^{\prime}, l \vdash Q_{0} \mid l\left[Q_{1}\right] \stackrel{\bar{a}\langle N\rangle}{\Longrightarrow}$ $t^{\prime}, r^{\prime}, l \vdash Q_{0}^{\prime} \mid l\left[Q_{1}^{\prime}\right]$, hence $t \vdash Q_{0}\left|Q_{1} \stackrel{\bar{a}\langle N\rangle}{\Longrightarrow} t^{\prime} \vdash Q_{0}^{\prime}\right| Q_{1}^{\prime}$ and also $\left(s, r^{\prime}, l \vdash\right.$ $\left.P_{0}\right) \mathcal{Y}_{(M, N) \oplus\left({ }^{(} P_{1}^{\prime},{ }^{\prime} Q_{1}^{\prime}\right) \oplus \mathcal{E} ; r l}^{-}\left(t^{\prime}, r^{\prime}, l \vdash Q_{0}^{\prime}\right)$, hence $\left(s \vdash P_{0} \mid P_{1}^{\prime}\right) \mathcal{Y}_{(M, N) \oplus \mathcal{E} ; r}^{-}\left(t^{\prime} \vdash\right.$ $\left.Q_{0}^{\prime} \mid Q_{1}^{\prime}\right)$ up-to context, environment and name creation.

Corollary B.38. [Output preserves run-erased environmental bisimulation upto context]

For any environmental bisimulation up-to context $\mathcal{Y}$, if $(s \vdash P) \mathcal{Y}_{\mathcal{E} ; r}^{-}(t \vdash Q)$ and $s \vdash P \stackrel{\bar{a}\langle M\rangle}{\longrightarrow} s \vdash P^{\prime}$ with $a \in r$, then there are $Q^{\prime}, t^{\prime}$ such that $t \vdash Q \stackrel{\bar{a}\langle N\rangle}{\longrightarrow} t^{\prime} \vdash Q^{\prime}$ and $\left(s \vdash P^{\prime}\right) \mathcal{Y}_{(M, N) \oplus \mathcal{E} ; r}^{-}\left(t^{\prime} \vdash Q^{\prime}\right)$. The converse on $Q$ 's transition holds too.

Proof. By $\mathcal{Y}^{-}$'s definition, we know there are $P^{+}, Q^{+}$and $\mathcal{E}^{+}$such that $(s \vdash$ $\left.P^{+}\right) \mathcal{Y}_{\mathcal{E}^{+} ; r}^{\star}\left(t \vdash Q^{+}\right)$. Since $s \vdash P \stackrel{\bar{a}\langle M\rangle}{\longrightarrow} s \vdash P^{\prime}$, there is a minimal output transition $s \vdash P^{+} \stackrel{\text { run }}{\Longrightarrow} \stackrel{\bar{a}\left\langle M^{+}\right\rangle}{\longrightarrow} s \vdash P^{\prime+}$. By Lemma B.37, we have $t \vdash Q^{+} \stackrel{\bar{a}\left\langle N^{+}\right\rangle}{\Longrightarrow} t^{\prime} \vdash Q^{\prime+}$ and $\left(s \vdash P^{\prime+}\right) \mathcal{Y}_{\left(M^{+}, N^{+}\right) \oplus \mathcal{E}^{+} ; r}^{-}\left(t^{\prime} \vdash Q^{\prime+}\right)$ which implies by Corollary B.23 that $t \vdash Q$ can also weakly do an output transition $t \vdash Q \stackrel{\bar{a}\langle N\rangle}{\Longrightarrow} t^{\prime} \vdash Q^{\prime}$, such that $Q^{\prime} \leq Q^{\prime+}$ and $N \leq N^{+}$. By Corollary B.29, as $P^{\prime} \leq P^{\prime+}, Q^{\prime} \leq Q^{\prime+}$ and $(M, N) \oplus \mathcal{E} \leq\left(M^{+}, N^{+}\right) \oplus \mathcal{E}^{+}$, we have $\left(s \vdash P^{\prime}\right) \mathcal{Y}_{(M, N) \oplus \mathcal{E} ; r}^{-}\left(t^{\prime} \vdash Q^{\prime}\right)$ as desired. Visually, the following diagram holds. 


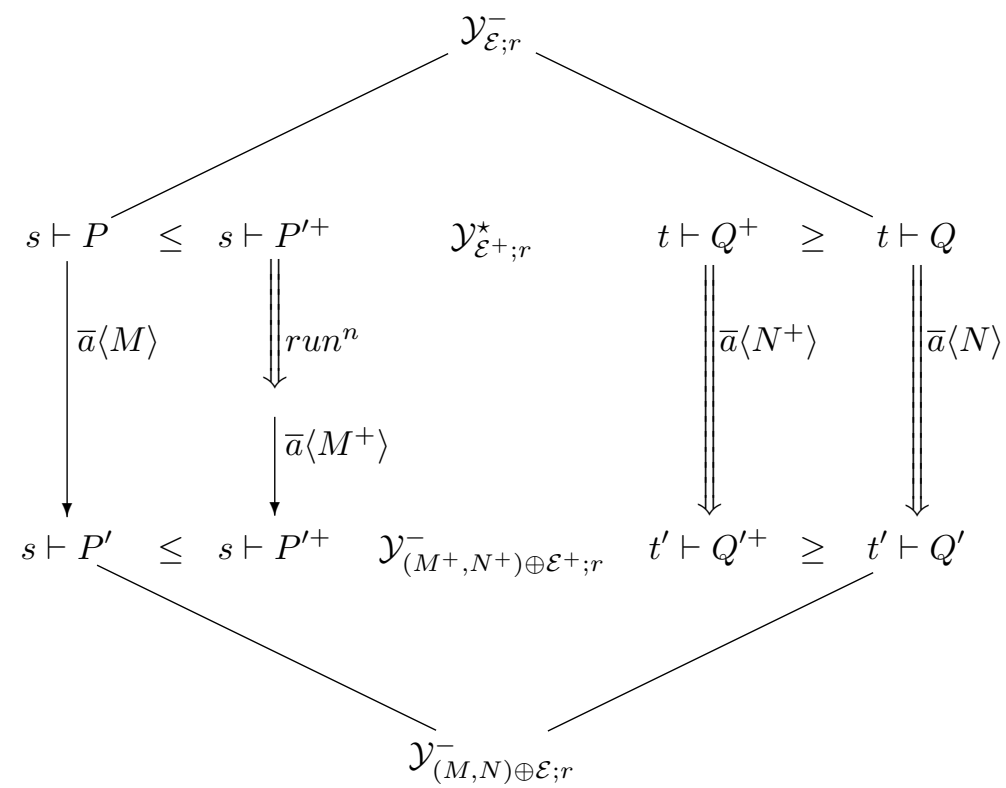

The converse on $Q$ 's transitions is shown similarly.

Lemma B.39. [run-expanded input]

Suppose that $(s \vdash P) \mathcal{Y}_{\mathcal{E} ; r}^{\star}(t \vdash Q)$ for an environmental bisimulation up-to context $\mathcal{Y}$ and that $s \vdash P \stackrel{\text { run nn }^{n}}{\longrightarrow} \stackrel{a(M)}{\longrightarrow} s \vdash P^{\prime}$ is minimal with respect to $s \vdash P^{-} \stackrel{a\left(M^{-}\right)}{\longrightarrow} s \vdash$ $P^{\prime-}$. Then for all $N$ such that $(M, N) \in(\mathcal{E} ; r)^{\star}, t \vdash Q \stackrel{a(N)}{\Longrightarrow} t^{\prime} \vdash Q^{\prime}$, and $\left(s \vdash P^{\prime}\right) \mathcal{Y}_{\mathcal{E} ; r}^{-}\left(t^{\prime} \vdash Q^{\prime}\right)$.

Proof. By induction on $n$.

- Case $n=0$

Immediate by Lemma B.15 and the fact that $\mathcal{Y}^{\star} \subseteq \mathcal{Y}^{-}$.

- Case $n>0$

By Lemma B.35 and Lemma B.26, we have two possible subcases preserving minimality after the first run-transition of $s \vdash P \stackrel{\text { run }^{n}}{\longrightarrow} \stackrel{a(M)}{\longrightarrow} s \vdash P^{\prime}$.

- Subcase $s \vdash P \stackrel{\text { run }}{\longrightarrow} s \vdash P^{\prime \prime}, t \vdash Q \stackrel{\tau}{\Rightarrow} t^{\prime \prime} \vdash Q^{\prime \prime},\left(s \vdash P^{\prime \prime}\right) \mathcal{Y}_{\mathcal{E}: r}^{\star}\left(t^{\prime \prime} \vdash Q^{\prime \prime}\right)$

We have that $s \vdash P^{\prime \prime} \stackrel{\text { run }^{n-1}}{\longrightarrow} \stackrel{a(M)}{\longrightarrow} s \vdash P^{\prime}$ is still minimal, so we can apply the induction hypothesis, and we are done.

- Subcase $s \vdash P \stackrel{\text { run }}{\longrightarrow} s \vdash P^{\prime \prime}, t \vdash Q \stackrel{\text { run }}{\longrightarrow} t \vdash Q^{\prime \prime},\left(s \vdash P^{\prime \prime}\right) \mathcal{Y}_{\mathcal{E}: r}^{-}\left(t \vdash Q^{\prime \prime}\right)$ with $P^{\prime \prime}=P_{0} \mid P_{1}$ and $Q^{\prime \prime}=Q_{0} \mid Q_{1}$, some $\mathcal{E}^{\prime}$ such that $\left(s, r^{\prime} \vdash P_{0}\right) \mathcal{Y}_{\mathcal{E}^{\prime} ; r r^{\prime}}\left(t, r^{\prime} \vdash\right.$ $\left.Q_{0}\right),\left(P_{1}, Q_{1}\right)=\left(C_{p}[\operatorname{run}(\widetilde{M}), A], C_{p}[\operatorname{run}(\widetilde{N}), B]\right)$ with $\left({ }^{\prime} A,{ }^{\prime} B\right) \in \mathcal{E}$ and $(A, B) \notin\left(\mathcal{E}^{\prime} ; r r^{\prime}\right)^{\circ},(\widetilde{M}, \widetilde{N}) \in \mathcal{E}^{\prime},\left(s, r^{\prime} \vdash P_{0}\right) \mathcal{Y}_{\mathcal{E}^{\prime} ; r r^{\prime}}\left(t, r^{\prime} \vdash Q_{0}\right)$ and $r^{\prime} \cap(s \cup t)=\emptyset$.

Using Lemma B.30 (to add a fresh $l$ to the know names) and clause 4 of environmental bisimulations up-to context, we have $\left(s, r^{\prime}, l \vdash P_{0} \mid\right.$ 
$l[A]) \mathcal{Y}_{\mathcal{E}^{\prime}: l \oplus r r^{\prime}}^{\star}\left(t, r^{\prime}, l \vdash Q_{0} \mid l[B]\right)$. Using an argument similar to the one in Lemma B.36, case 2, subcase 2, we know that we can apply the induction hypothesis to minimal transition $s, r^{\prime}, l \vdash P_{0} \mid l[A] \stackrel{\text { run }^{n-1}}{\longrightarrow} \stackrel{a(M)}{\longrightarrow} s, r^{\prime}, l \vdash$ $P_{0} \mid l\left[A^{\prime}\right]$. We obtain $t, r^{\prime}, l \vdash Q_{0}\left|l[B] \stackrel{a(N)}{\Longrightarrow} t^{\prime \prime}, r^{\prime}, l \vdash Q_{0}^{\prime \prime}\right| l\left[B^{\prime \prime}\right]$ and $\left(s, r^{\prime}, l \vdash P_{0} \mid l\left[A^{\prime}\right]\right) \mathcal{Y}_{\mathcal{E}^{\prime} ; l \oplus r r^{\prime}}^{-}\left(t^{\prime \prime}, r^{\prime}, l \vdash Q_{0}^{\prime \prime} \mid l\left[B^{\prime \prime}\right]\right)$. By Corollary B.38, after an output to channel $l$, we have $\left(s, r^{\prime}, l \vdash P_{0}\right) \mathcal{Y}_{\left(A^{\prime}, B^{\prime}\right) \oplus \mathcal{E}^{\prime} ; l \oplus r r^{\prime}}^{-}\left(t^{\prime}, r^{\prime}, l \vdash\right.$ $\left.Q_{0}^{\prime}\right)$, hence $\left(s \vdash P_{0} \mid C_{p}\left[\operatorname{run}(\widetilde{M}), A^{\prime}\right]\right) \mathcal{Y}_{\mathcal{E} ; r}^{-}\left(t^{\prime} \vdash Q_{0}^{\prime} \mid C_{p}\left[\operatorname{run}(\widetilde{N}), B^{\prime}\right]\right)$ upto environment, context and name creation. And of course, we do have $t \vdash Q \stackrel{a(N)}{\Longrightarrow} t^{\prime} \vdash Q_{0}^{\prime} \mid C_{p}\left[\operatorname{run}(\widetilde{N}), B^{\prime}\right]$.

Corollary B.40. [Input preserves run-erased environmental bisimulation upto context]

For any environmental bisimulation up-to context $\mathcal{Y}$, if $(s \vdash P) \mathcal{Y}_{\mathcal{E} ; r}^{-}(t \vdash Q)$ and $s \vdash P \stackrel{a(M)}{\longrightarrow} s \vdash P^{\prime}$ with $a \in r$, then there are $t^{\prime}, Q^{\prime}$ such that for all $(M, N) \in(\mathcal{E} ; r)^{-}, t \vdash Q \stackrel{a(N)}{\Longrightarrow} t^{\prime} \vdash Q^{\prime}$ and $\left(s \vdash P^{\prime}\right) \mathcal{Y}_{\mathcal{E} ; r}^{-}\left(t^{\prime} \vdash Q^{\prime}\right)$. The converse on $t \vdash Q$ 's transitions holds too.

Proof. By $\mathcal{Y}^{-}$'s definition, we know there are $s \vdash P^{+}, t \vdash Q^{+}$and $\mathcal{E}^{+}$such that $\left(s \vdash P^{+}\right) \mathcal{Y}_{\mathcal{E}^{+} ; r}^{\star}\left(t \vdash Q^{+}\right)$. Since $s \vdash P \stackrel{a(M)}{\longrightarrow} s \vdash P^{\prime}$, there is a minimal input transition $s \vdash P^{+} \stackrel{\text { run }}{\longrightarrow} \stackrel{a\left(M^{+}\right)}{\longrightarrow} s \vdash P^{\prime+}$. By Lemma B.39, we have $t \vdash Q^{+} \stackrel{a\left(N^{+}\right)}{\Longrightarrow} t \vdash Q^{\prime+}$ for any $\left(M^{+}, N^{+}\right) \in\left(\mathcal{E}^{+} ; r\right)^{\star}$ and $\left(s \vdash P^{\prime+}\right) \mathcal{Y}_{\mathcal{E}^{+} ; r}^{-}\left(t \vdash Q^{\prime+}\right)$ which implies by Corollary B.23 that $t \vdash Q$ can also weakly do an input transition $t \vdash Q \stackrel{a(N)}{\Longrightarrow} t^{\prime} \vdash Q^{\prime}$ such that $Q^{\prime} \leq Q^{\prime+}$ for any $N \leq N^{+}$, i.e. for any $(M, N) \in(\mathcal{E} ; r)^{-}$. By Corollary B.29, as $P^{\prime} \leq P^{\prime+}, Q^{\prime} \leq Q^{\prime+}$ and $\mathcal{E} \leq \mathcal{E}^{+}$, we have $\left(s \vdash P^{\prime}\right) \mathcal{Y}_{\mathcal{E} ; r}^{-}\left(t^{\prime} \vdash Q^{\prime}\right)$ as desired. Visually, the following diagram holds. 


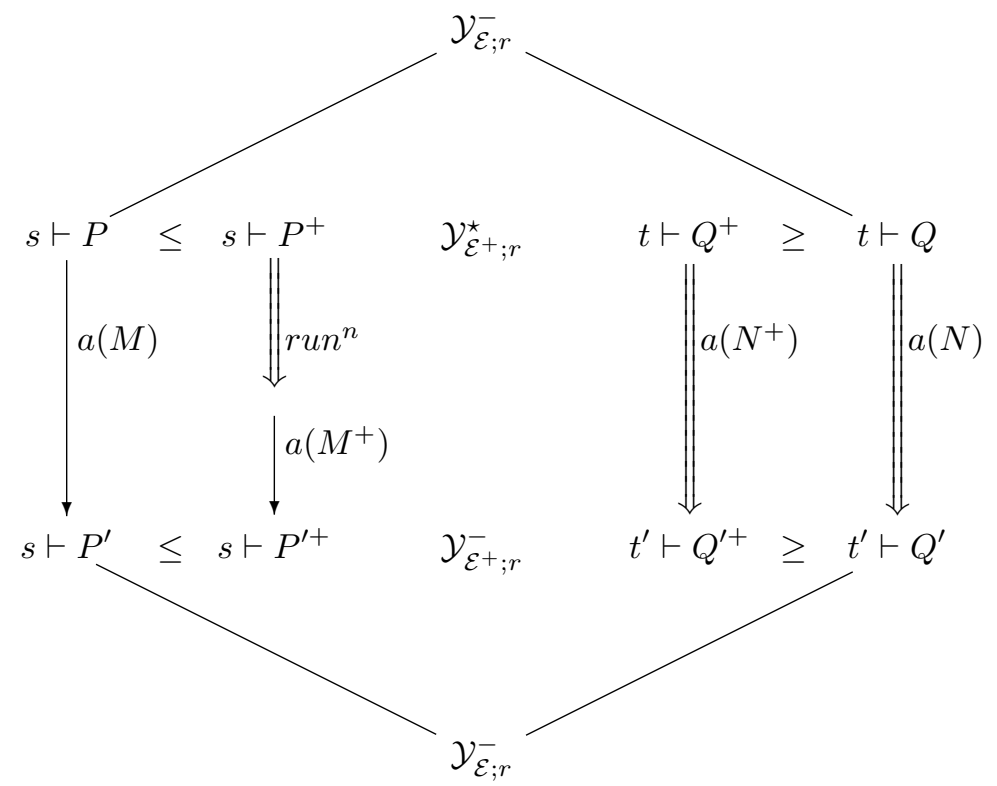

The converse on $t \vdash Q$ 's transitions is shown similarly.

Lemma B.41. [run-expanded reduction for environmental bisimulation up-to context]

Suppose that $(s \vdash P) \mathcal{Y}_{\mathcal{E} ; r}^{\star}(t \vdash Q)$ for an environmental bisimulation up-to context $\mathcal{Y}$, and that $s \vdash P \stackrel{\text { run }^{n}}{\longrightarrow} \stackrel{\tau}{\rightarrow} s^{\prime} \vdash P^{\prime}$ is minimal with respect to $s \vdash P^{-} \stackrel{\tau}{\rightarrow} s^{\prime} \vdash P^{\prime-}$, then $t \vdash Q \stackrel{\tau}{\Rightarrow} t^{\prime} \vdash Q^{\prime}$, and $\left(s^{\prime} \vdash P^{\prime}\right) \mathcal{Y}_{\mathcal{E} ; r}^{-}\left(t^{\prime} \vdash Q^{\prime}\right)$.

Proof. By induction on $n$.

- Case $n=0$

Immediate by Lemma B.35.

- Case $n>0$

By Lemma B.35 and Lemma B.26, we have two possible subcases preserving minimality after the first run-transition of $s \vdash P \stackrel{\text { run }^{n}}{\longrightarrow} \stackrel{\tau}{\rightarrow} s \vdash P^{\prime}$.

- Subcase $s \vdash P \stackrel{\text { run }}{\longrightarrow} s \vdash P^{\prime \prime}, t \vdash Q \stackrel{\tau}{\Rightarrow} t^{\prime \prime} \vdash Q^{\prime \prime},\left(s \vdash P^{\prime \prime}\right) \mathcal{Y}_{\mathcal{E} ; r}^{\star}\left(t^{\prime \prime} \vdash Q^{\prime \prime}\right)$

We have that $s \vdash P^{\prime \prime} \stackrel{\text { run nn-1 }^{\longrightarrow}}{\longrightarrow} \stackrel{\tau}{\rightarrow} \vdash P^{\prime}$ is still minimal with respect to $s \vdash P^{-} \stackrel{\tau}{\rightarrow} s \vdash P^{\prime-}$, so we can apply the induction hypothesis, and we are done.

- Subcase $s \vdash P \stackrel{\text { run }}{\longrightarrow} s \vdash P^{\prime \prime}, t \vdash Q \stackrel{\text { run }}{\longrightarrow} t \vdash Q^{\prime \prime},\left(s \vdash P^{\prime \prime}\right) \mathcal{Y}_{\mathcal{E} ; r}^{-}\left(t \vdash Q^{\prime \prime}\right)$ hold, and we have $P^{\prime \prime}=P_{0} \mid P_{1}$ and $Q^{\prime \prime}=Q_{0} \mid Q_{1}$ with $\left(s, r^{\prime} \vdash P_{0}\right) \mathcal{Y}_{\mathcal{E}^{\prime} ; r r^{\prime}}$ $\left(t, r^{\prime} \vdash Q_{0}\right),\left({ }^{\prime} P_{1},{ }^{\prime} Q_{1}\right)=\left({ }^{\prime} C_{p}[\operatorname{run}(\widetilde{M}), A],{ }^{\prime} C_{p}[\operatorname{run}(\widetilde{N}), B]\right) \in\left(\mathcal{E}^{\prime} ; r r^{\prime}\right)^{-}$ with $(A, B) \notin\left(\mathcal{E}^{\prime} ; r r^{\prime}\right)^{\circ},\left((\widetilde{M} ; ' A),\left(\widetilde{N},{ }^{\prime} B\right)\right) \in \mathcal{E}^{\prime}, r^{\prime} \cap(s \cup t)=\emptyset$. Since we know $s, r^{\prime} \vdash P^{-} \stackrel{\tau}{\rightarrow} s^{\prime}, r^{\prime} \vdash P^{\prime-}$ and that $s, r^{\prime} \vdash P \stackrel{\text { run }}{\longrightarrow} \stackrel{\tau}{\rightarrow} s^{\prime}, r^{\prime} \vdash P^{\prime}$ is minimal with respect to it, we can infer how $P$ weakly reduces to $P^{\prime}$. Let us analyse each possibility. 
* Subsubcase $A$ reacts with $P_{0}$

Using clause 5 of environmental bisimulation up-to context to add a new name $l$ to known names, and clause 4 to spawn $A$, we have $\left(s, l, r^{\prime} \vdash P_{0} \mid l[A]\right) \mathcal{Y}_{\mathcal{E}^{\prime} ; r r^{\prime} l}^{\star}\left(t, l, r^{\prime} \vdash Q_{0} \mid l[B]\right)$. Using an argument similar to the one in Lemma B.36, case 2, subcase 2, we know that we can apply the induction hypothesis to minimal transition $s, r^{\prime}, l \vdash P_{0} \mid$ $l[A] \stackrel{\text { run }{ }^{n-1}}{\longrightarrow} \stackrel{\tau}{\rightarrow} s^{\prime}, r^{\prime}, l \vdash P_{0}^{\prime} \mid l\left[A^{\prime}\right]$. We obtain $t, r^{\prime}, l \vdash Q_{0} \mid l[B] \stackrel{\tau}{\Rightarrow} t^{\prime \prime}, r^{\prime}, l \vdash$ $Q_{0}^{\prime \prime} \mid l\left[B^{\prime \prime}\right]$ and $\left(s^{\prime}, r^{\prime}, l \vdash P_{0}^{\prime} \mid l\left[A^{\prime}\right]\right) \mathcal{Y}_{\mathcal{E}^{\prime} ; r r^{\prime} l}^{-}\left(t^{\prime \prime}, r^{\prime}, l \vdash Q_{0}^{\prime \prime} \mid l\left[B^{\prime \prime}\right]\right)$. Therefore, by $\left(s^{\prime}, r^{\prime}, l \vdash P_{0}^{\prime} \mid l\left[A^{\prime}\right]\right) \mathcal{Y}_{\mathcal{E}^{\prime} ; r r^{\prime} l}^{-}\left(t^{\prime \prime}, r^{\prime}, l \vdash Q_{0}^{\prime \prime} \mid l\left[B^{\prime \prime}\right]\right)$ and by Corollary B.38, we have $\left(s^{\prime}, r^{\prime}, l \vdash P_{0}^{\prime}\right) \mathcal{Y}_{\left({ }^{\circ} A^{\prime},{ }^{\prime} B^{\prime}\right) \oplus \mathcal{E}^{\prime} ; r r^{\prime} l}^{-}\left(t^{\prime}, r^{\prime}, l \vdash Q_{0}^{\prime}\right)$ hence $\left(s^{\prime}, r^{\prime}, l \vdash P_{0}^{\prime} \mid C_{p}\left[\operatorname{run}(\widetilde{M}), A^{\prime}\right]\right) \mathcal{Y}_{\left({ }^{\prime} A^{\prime},{ }^{\prime} B^{\prime}\right) \oplus \mathcal{E}^{\prime} ; r r^{\prime} l}^{-}\left(t^{\prime}, r^{\prime}, l \vdash Q_{0}^{\prime} \mid\right.$ $\left.C_{p}\left[\operatorname{run}(\widetilde{N}), B^{\prime}\right]\right)$ up-to context, $\left(s^{\prime} \vdash P_{0}^{\prime} \mid C_{p}\left[\operatorname{run}(\widetilde{M}), A^{\prime}\right]\right) \mathcal{Y}_{\mathcal{E} ; r}^{-}\left(t^{\prime} \vdash\right.$ $\left.Q_{0}^{\prime} \mid C_{p}\left[\operatorname{run}(\widetilde{N}), B^{\prime}\right]\right)$ up-to environment and name creation. Also, $t, r^{\prime}, l \vdash Q^{\prime \prime} \stackrel{\tau}{\Rightarrow} t^{\prime}, r^{\prime}, l \vdash Q_{0}^{\prime} \mid C_{p}\left[\tilde{N}, B^{\prime}\right]$, holds as well from the above, hence $t \vdash Q \stackrel{\tau}{\Rightarrow} t^{\prime} \vdash Q_{0}^{\prime} \mid C_{p}\left[\right.$ run $\left.(\widetilde{N}), B^{\prime}\right]$.

* Subsubcase $A$ reduces alone

Similarly, but with $P_{0}^{\prime}=P_{0}$.

* Subsubcase $A$ reacts with an $A_{i}$ from $' \widetilde{A}=\widetilde{M}$ (or a run-erasure of it)

Let $G=C\left[^{[} A_{i}, \widetilde{M^{\prime}}\right]$ (resp. $H=C\left[^{[} B_{i}, \widetilde{N}^{\prime}\right]$ ) be the process of $P_{1}$ (resp. $Q_{1}$ ) in redex position that contains $A_{i}$ (resp. $B_{i}$ ) and reacts with the process containing $A$ according to rule REACT-R or REACT$\mathrm{L}$. Then there is a process context $C_{p}^{\prime}$ such that $C_{p}[A, \operatorname{run}(\widetilde{M})]=$ $C_{p}^{\prime}\left[A^{\prime}, G, \operatorname{run}\left(\widetilde{M^{\prime \prime}}\right)\right]$.

By clause 5 of environmental bisimulation up-to context to add a new name $l$ and clause 4 , we have $\left(s, r^{\prime}, l \vdash P_{0} \mid l[A]\right) \mathcal{Y}_{\mathcal{E}^{\prime} ; r r^{\prime} l}^{\star}\left(t, r^{\prime}, l \vdash Q_{0} \mid\right.$ $l[B])$. By Lemma B.30 to add a new name $m$ and by up-to context, we have $\left(s, r^{\prime}, l, m \vdash P_{0}|l[A]| m[G]\right) \mathcal{Y}_{\mathcal{E}^{\prime} ; m \oplus r r^{\prime} l}^{\star}\left(t, r^{\prime}, l, m \vdash Q_{0}|l[B]| m[H]\right)$. Then, $s, r^{\prime}, l, m \vdash P_{0}|l[A]| m[G] \stackrel{\text { run }}{\longrightarrow} \stackrel{\tau}{\rightarrow} s, r^{\prime}, l, m \vdash P_{0}\left|l\left[A^{\prime}\right]\right| m\left[G^{\prime}\right]$. Applying the induction hypothesis, we obtain $t, r^{\prime}, l, m \vdash Q_{0}|l[B]|$ $m[H] \stackrel{\tau}{\Rightarrow} t^{\prime \prime}, r^{\prime}, l, m \vdash Q_{0}^{\prime \prime}\left|l\left[B^{\prime \prime}\right]\right| m\left[H^{\prime \prime}\right]$, as well as $\left(s, r^{\prime}, l, m \vdash P_{0} \mid\right.$ $\left.l\left[A^{\prime}\right] \mid m\left[G^{\prime}\right]\right) \mathcal{Y}_{\mathcal{E}^{\prime} ; m \oplus r r^{\prime} l}^{-}\left(t^{\prime \prime}, r^{\prime}, l, m \vdash Q_{0}^{\prime}\left|l\left[B^{\prime \prime}\right]\right| m\left[H^{\prime \prime}\right]\right)$.

We can now passivate the contents of $l[]$ and $m[]$ and use up-to context, environment and name creation to get $\left(s \vdash P_{0} \mid C_{p}^{\prime}\left[A^{\prime}, G^{\prime}, \operatorname{run}\left(\widetilde{M}^{\prime \prime}\right)\right]\right) \mathcal{Y}_{\mathcal{E} ; r}^{-}$ $\left(t^{\prime} \vdash Q_{0}^{\prime} \mid C_{p}^{\prime}\left[B^{\prime}, H^{\prime}, \operatorname{run}\left(\widetilde{N}^{\prime \prime}\right)\right]\right)$. It also follows that $t \vdash Q \stackrel{\tau}{\Rightarrow} t^{\prime} \vdash$ $Q_{0}^{\prime} \mid C_{p}^{\prime}\left[B^{\prime}, H^{\prime}, \operatorname{run}\left(\widetilde{N}^{\prime \prime}\right)\right]$ as expected.

* Subsubcase $A$ outputs and reacts with the context

Let $C\left[\widetilde{M}^{\prime}\right]\left(\right.$ resp. $\left.C\left[\widetilde{N}^{\prime}\right]\right)$ be the process of $P_{1}$ (resp. $\left.Q_{1}\right)$ in redex position that reacts with the process containing $A$ according to rule REACT-R or REACT-L. Then there is another context $C_{p}^{\prime}$ such that $C_{p}[A, \operatorname{run}(\widetilde{M})]=C_{p}^{\prime}\left[A, C\left[\widetilde{M}^{\prime}\right], \operatorname{run}\left(\widetilde{M}^{\prime \prime}\right)\right]$. Using clause 5 of environmental bisimulation up-to context to add a new name $l$ and clause 4 
to spawn $A$, we have $\left(s, r^{\prime}, l \vdash P_{0} \mid l[A]\right) \mathcal{Y}_{\mathcal{E}^{\prime} ; r r^{\prime} l}^{\star}\left(t, r^{\prime}, l \vdash Q_{0} \mid l[B]\right)$. We can now apply Lemma B.37 to simulate $A$ 's output after runtransitions: $s, r^{\prime}, l \vdash P_{0}\left|l[A] \stackrel{r u n^{n-1}}{\Longrightarrow} \stackrel{\bar{a}\langle M\rangle}{\longrightarrow} s, r^{\prime}, l \vdash P_{0}\right| l\left[A^{\prime}\right]$, and we obtain $t, r^{\prime}, l \vdash Q_{0}\left|l[B] \stackrel{\bar{a}\langle N\rangle}{\Longrightarrow} t^{\prime \prime}, r^{\prime}, l \vdash Q_{0}^{\prime \prime}\right| l\left[B^{\prime \prime}\right]$, and $\left(s, r^{\prime}, l \vdash P_{0} \mid\right.$ $\left.l\left[A^{\prime}\right]\right) \mathcal{Y}_{(M, N) \oplus \mathcal{E}^{\prime} ; r r^{\prime} l}^{-}\left(t^{\prime \prime}, r^{\prime}, l \vdash Q_{0}^{\prime \prime} \mid l\left[B^{\prime \prime}\right]\right)$. We passivate the contents of $l[]$ and have $\left(s, r^{\prime}, l \vdash P_{0}\right) \mathcal{Y}_{\left({ }^{-} A^{\prime},{ }^{\prime} B^{\prime}\right) \oplus(M, N) \oplus \mathcal{E}^{\prime} ; r r^{\prime} l}\left(t^{\prime}, r^{\prime}, l \vdash Q_{0}^{\prime}\right)$. By Lemma B.12, we have that $s, r^{\prime}, l \vdash C[\widetilde{M}] \stackrel{a(M)}{\longrightarrow} s, r^{\prime}, l \vdash G$ and $t^{\prime \prime \prime}, r^{\prime}, l \vdash C[\tilde{N}] \stackrel{a(N)}{\longrightarrow} t^{\prime \prime \prime}, r^{\prime}, l \vdash H$ with $(G, H) \in\left((M, N) \oplus \mathcal{E}^{\prime} ; r r^{\prime} l\right)^{\circ}$ (for some "intermediate" $t^{\prime \prime \prime}$ ) such that we have $t^{\prime \prime}, r^{\prime}, l \vdash Q_{0}^{\prime \prime}\left|l\left[B^{\prime \prime}\right]\right|$ $C[\widetilde{N}] \stackrel{\tau}{\Rightarrow} t^{\prime \prime \prime}, r^{\prime}, l \vdash Q_{0}^{\prime \prime \prime}\left|l\left[B^{\prime \prime \prime}\right]\right| C[\widetilde{N}] \stackrel{\tau}{\rightarrow} t^{\prime \prime \prime}, r^{\prime}, l \vdash Q_{0}^{\prime \prime \prime \prime}\left|l\left[B^{\prime \prime \prime \prime}\right]\right| H \stackrel{\tau}{\Rightarrow}$ $t^{\prime}, r^{\prime}, l \vdash Q_{0}^{\prime}\left|l\left[B^{\prime}\right]\right| H$ hence $t \vdash Q_{0}\left|Q_{1} \stackrel{\tau}{\Rightarrow} t^{\prime} \vdash Q_{0}^{\prime}\right| Q_{1}^{\prime}$. From $\left(s, r^{\prime}, l \vdash\right.$ $\left.P_{0}\right) \mathcal{Y}_{\left({ }^{\prime} A^{\prime},{ }^{\prime} B^{\prime}\right) \oplus(M, N) \oplus \mathcal{E}^{\prime} ; r r^{\prime} l}\left(t^{\prime}, r^{\prime}, l \vdash Q_{0}^{\prime}\right)$. we build up-to context, environment and name creation $\left(s \vdash P_{0} \mid C_{p}^{\prime}\left[A^{\prime}, G, \operatorname{run}(\widetilde{M})\right]\right) \mathcal{Y}_{\mathcal{E} ; r}^{-}\left(t^{\prime} \vdash\right.$ $\left.Q_{0}^{\prime} \mid C_{p}\left[B^{\prime}, H, \operatorname{run}(\tilde{N})\right]\right)$.

* Subsubcase $A$ inputs and reacts with the context

Suppose the context outputs a process $M$ (resp. $N$ by Lemma B.13) by means of a process $C_{o}\left[\widetilde{M}^{\prime}\right]$ (resp. $\left.C_{o}\left[\widetilde{N}^{\prime}\right]\right)$. Using clause 5 of environmental bisimulation up-to context several times to add a new name $l$ and clause 4 to spawn $A$, we have $\left(s, r^{\prime}, l \vdash P_{0} \mid l[A]\right) \mathcal{Y}_{\mathcal{E}^{\prime} ; r r^{\prime} l}^{\star}$ $\left(t, r^{\prime}, l \vdash Q_{0} \mid l[B]\right)$. We can now apply Lemma B.39 to trigger $A$ 's input of $M$ and $Q_{0} \mid l[B]$ 's simulation of that input, and we obtain $\left(s, r^{\prime}, l \vdash P_{0} \mid l\left[A^{\prime}\right]\right) \mathcal{Y}_{\mathcal{E}^{\prime} ; r r^{\prime} l}^{-}\left(t^{\prime \prime}, r^{\prime}, l \vdash Q_{0}^{\prime \prime} \mid l\left[B^{\prime \prime}\right]\right)$. We passivate the content of $l[]$, obtaining $\left(s, r^{\prime}, l \vdash P_{0}\right) \mathcal{Y}_{\left({ }^{\prime} A^{\prime},{ }^{\prime} B^{\prime}\right) \oplus \mathcal{E}^{\prime} ; r r^{\prime} l}\left(t^{\prime}, r^{\prime}, l \vdash Q_{0}^{\prime}\right)$. We remove $r^{\prime}$ and $l$ up-to name creation from the known names and then replace $A^{\prime}$ and $B^{\prime}$ up-to context, giving $\left(s \vdash P_{0} \mid C_{p}^{\prime}\left[A^{\prime}, \operatorname{run}(\widetilde{M})\right]\right) \mathcal{Y}_{\left({ }^{-} A^{\prime},{ }^{\prime} B^{\prime}\right) \oplus \mathcal{E}^{\prime} ; r}^{-}$ $\left(t^{\prime} \vdash Q_{0}^{\prime} \mid C_{p}^{\prime}\left[B^{\prime}, \operatorname{run}(\tilde{N})\right]\right)$. Finally, up-to environment, we have $(s \vdash$ $\left.P_{0} \mid C_{p}^{\prime}\left[A^{\prime}, \operatorname{run}(\widetilde{M})\right]\right) \mathcal{Y}_{\mathcal{E} ; r}^{-}\left(t^{\prime} \vdash Q_{0}^{\prime} \mid C_{p}\left[B^{\prime}, \operatorname{run}(\widetilde{N})\right]\right)$. The transition $t \vdash Q_{0}\left|Q_{1} \stackrel{\tau}{\Rightarrow} t^{\prime} \vdash Q_{0}^{\prime}\right| C_{p}\left[B^{\prime}, \operatorname{run}(\widetilde{N})\right]$ is derived from the above, composing input $t \vdash Q_{0}\left|Q_{1} \stackrel{\tau}{\Rightarrow} \stackrel{a(N)}{\longrightarrow} \stackrel{\tau}{\Rightarrow} t^{\prime} \vdash Q_{0}^{\prime}\right| C_{p}\left[B^{\prime}, \operatorname{run}(\tilde{N})\right]$ with the output from the context.

Corollary B.42. [Reduction preserves run-erased environmental bisimulation up-to context]

For any environmental bisimulation up-to context $\mathcal{Y}$, if $(s \vdash P) \mathcal{Y}_{\mathcal{E} ; r}^{-}(t \vdash Q)$ and $s \vdash P \stackrel{\tau}{\rightarrow} s^{\prime} \vdash P^{\prime}$, then there are $t^{\prime}$ and $Q^{\prime}$ such that $t \vdash Q \stackrel{\tau}{\Rightarrow} t^{\prime} \vdash Q^{\prime}$ and $\left(s^{\prime} \vdash P^{\prime}\right) \mathcal{Y}_{\mathcal{E} ; r}^{-}\left(t^{\prime} \vdash Q^{\prime}\right)$. The converse on $t \vdash Q$ 's transitions holds too.

Proof. By $\mathcal{Y}^{-}$'s definition, we know there are $s \vdash P^{+}, t \vdash Q^{+}$and $\mathcal{E}^{+}$such that $\left(s \vdash P^{+}\right) \mathcal{Y}_{\mathcal{E}^{+} ; r}^{\star}\left(t \vdash Q^{+}\right)$. Since $s \vdash P \stackrel{\tau}{\rightarrow} s^{\prime} \vdash P^{\prime}$, there is a minimal reduction transition $s \vdash P^{+} \stackrel{\text { run }}{\Longrightarrow} \stackrel{\tau}{\rightarrow} s^{\prime} \vdash P^{\prime+}$. By Lemma B.41, we have $t \vdash Q^{+} \stackrel{\tau}{\Rightarrow} t^{\prime} \vdash Q^{\prime+}$ and $\left(s^{\prime} \vdash P^{\prime+}\right) \mathcal{Y}_{\mathcal{E}^{+} ; r}^{-}\left(t^{\prime} \vdash Q^{\prime+}\right)$ which implies by Corollary B.23 that $t \vdash Q$ can 
also weakly reduce to some $t^{\prime} \vdash Q^{\prime}$ such that $Q^{\prime} \leq Q^{\prime+}$. By Corollary B.29, as $P^{\prime} \leq P^{\prime+}, Q^{\prime} \leq Q^{\prime+}$ and $\mathcal{E} \leq \mathcal{E}^{+}$, we have $\left(s^{\prime} \vdash P^{\prime}\right) \mathcal{Y}_{\mathcal{E} ; r}^{-}\left(t^{\prime} \vdash Q^{\prime}\right)$ as desired. Visually, the following diagram holds.

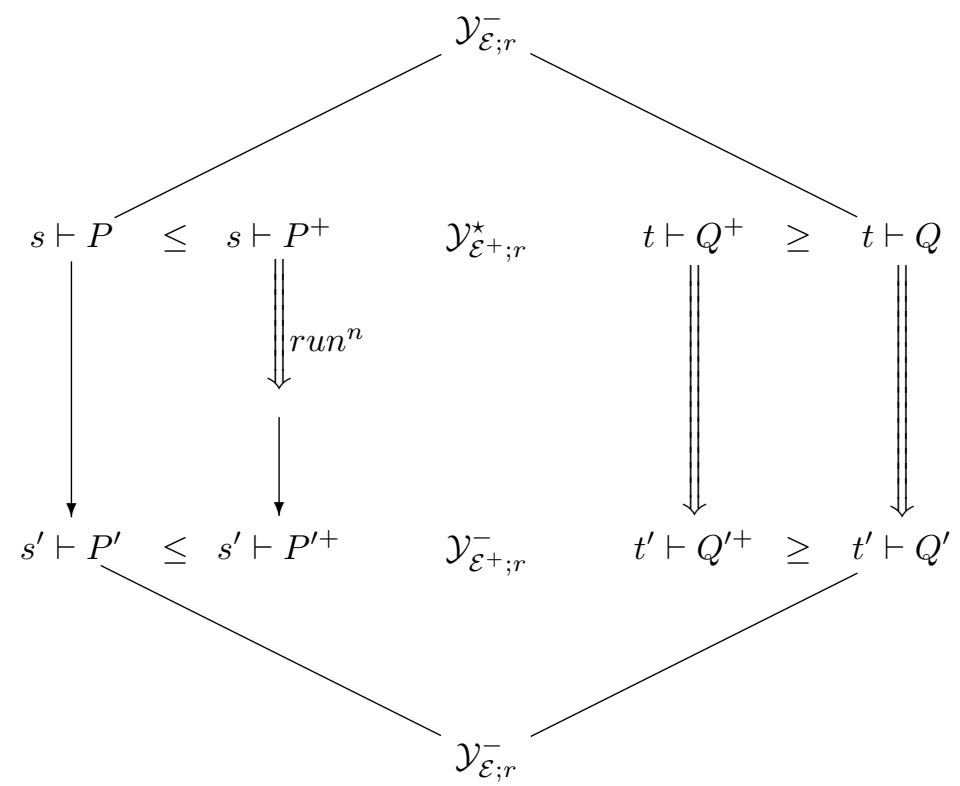

The converse on $t \vdash Q$ 's transitions is shown similarly.

Theorem B.43. [Soundness of environmental bisimulation up-to context]

If $\mathcal{Y}$ is a environmental bisimulation up-to context, then $\mathcal{Y}^{-}$is included in bisimilarity.

Proof. Let $\mathcal{X}=\left\{(\mathcal{E}, r, s, P, t, Q) \mid(s \vdash P) \mathcal{Y}_{\mathcal{E} ; r}^{-}(t \vdash Q)\right\}$ and let us prove that $\mathcal{X}$ verifies each clause of environmental bisimulation.

1. By Corollary B.42, whenever $s \vdash P \stackrel{\tau}{\rightarrow} s^{\prime} \vdash P^{\prime}$, we have a $t^{\prime} \vdash Q^{\prime}$ such that $t \vdash Q \stackrel{\tau}{\Rightarrow} t^{\prime} \vdash Q^{\prime}$ and $\left(s \vdash P^{\prime}\right) \mathcal{Y}_{\mathcal{E} ; r}^{-}\left(t^{\prime} \vdash Q^{\prime}\right)$, i.e. $\left(s \vdash P^{\prime}\right) \mathcal{X}_{\mathcal{E} ; r}\left(t^{\prime} \vdash Q^{\prime}\right)$.

2. By Corollary B.38, whenever $s \vdash P \stackrel{\bar{a}\langle M\rangle}{\longrightarrow} s \vdash P^{\prime}$ with $a \in r$, we have a $t^{\prime} \vdash Q^{\prime}$ such that $t \vdash Q \stackrel{\bar{a}\langle N\rangle}{\Longrightarrow} t^{\prime} \vdash Q^{\prime}$ and $\left(s \vdash P^{\prime}\right) \mathcal{Y}_{(M, N) \oplus \mathcal{E} ; r}^{-}\left(t^{\prime} \vdash Q^{\prime}\right)$, i.e. $\left(s \vdash P^{\prime}\right) \mathcal{X}_{(M, N) \oplus \mathcal{E} ; r}\left(t^{\prime} \vdash Q^{\prime}\right)$.

3. By Corollary B.40, whenever $s \vdash P \stackrel{a(M)}{\longrightarrow} s \vdash P^{\prime}$ with $a \in r$, we have for all $(M, N) \in(\mathcal{E} ; r)^{\star}$ a $t^{\prime} \vdash Q^{\prime}$ such that $t \vdash Q \stackrel{a(N)}{\longrightarrow} t^{\prime} \vdash Q^{\prime}$ with $\left(s \vdash P^{\prime}\right) \mathcal{Y}_{\mathcal{E} ; r}^{-}\left(t^{\prime} \vdash\right.$ $\left.Q^{\prime}\right)$, i.e. $\left(s \vdash P^{\prime}\right) \mathcal{X}_{\mathcal{E} ; r}\left(t^{\prime} \vdash Q^{\prime}\right)$.

4. By Lemma B.31, we have $\left(s \vdash P^{+} \mid l\left[P_{1}^{+}\right]\right) \mathcal{Y}_{\mathcal{E}^{+} ; r}^{\star}\left(t \vdash Q^{+} \mid l\left[Q_{1}^{+}\right]\right)$for some $(s \vdash$ $\left.P^{+}\right) \mathcal{Y}_{\mathcal{E}^{+} ; r}^{\star}\left(t \vdash Q^{+}\right)$with $P \leq P^{+}, Q \leq Q^{+}, \mathcal{E} \subseteq \leq \mathcal{E}^{+} \geq$, and $\left({ }^{\prime} P_{1},{ }^{\prime} Q_{1}\right) \leq$ $\left({ }^{\prime} P_{1}^{+},{ }^{\prime} Q_{1}^{+}\right) \in \mathcal{E}^{+}$, whose existence is guaranteed by definition of $\mathcal{Y}^{-}$. Then, by run-erasure, we have $\left(s \vdash P \mid l\left[P_{1}\right]\right) \mathcal{Y}_{\mathcal{E} ; r}^{-}\left(t \vdash Q \mid l\left[Q_{1}\right]\right)$. 
5. By Lemma B.30, we have for any $n$ not in $s \cup t,(s, n \vdash P) \mathcal{Y}_{\mathcal{E} ; n \oplus r}^{-}(t, n \vdash Q)$, i.e. $(s, n \vdash P) \mathcal{X}_{\mathcal{E} ; n \oplus r}(t, n \vdash Q)$.

6. Similarly, the converse of the first three clauses holds too.

Theorem B.44. [Reduction-closed Barbed equivalence from environmental bisimulation]

Let $r \subseteq f=f n(P, Q)$, then if $(f \vdash P) \mathcal{Y}_{\emptyset: r}^{-}(f \vdash Q)$ for an environmental bisimulation up-to context $\mathcal{Y}$, then $f \vdash P \approx_{r} f \vdash Q$.

Proof. We know by Theorem B.43 that $\mathcal{Y}^{-}$is an environmental bisimulation. We let $\mathcal{Z}=\left\{(r, s, P, t, Q) \mid f n(P, Q) \subseteq s \cap t, \quad r \subseteq s \cap t, \quad(s \vdash P) \mathcal{Y}_{\emptyset ; r}^{-}(t \vdash Q)\right\}$ and prove that $\mathcal{Z}$ is included in $\approx$.

1. Clause $s \vdash P \stackrel{\tau}{\rightarrow} s^{\prime} \vdash P$

As $\mathcal{Y}^{-}$is an environmental bisimulation, by clause 1 of the bisimulation, there is $t^{\prime} \vdash Q^{\prime}$ such that $t \vdash Q \Rightarrow t^{\prime} \vdash Q^{\prime}$ and $\left(s^{\prime} \vdash P^{\prime}\right) \mathcal{Y}_{\emptyset ; r}^{-}\left(t^{\prime} \vdash Q^{\prime}\right)$. We have $f n\left(P^{\prime}, Q^{\prime}\right) \subseteq s^{\prime} \cap t^{\prime}$ and $r \subseteq s^{\prime} \cap t^{\prime}$, henc $\left(r, s^{\prime}, P^{\prime}, t^{\prime}, Q^{\prime}\right) \in \mathcal{Z}$.

2. Clause $P \downarrow_{\mu} \quad \mu$ or $\bar{\mu} \in r$

There are two cases depending on $\mu$ :

- Case $s \vdash P \downarrow_{a}$

We have that $s \vdash P \stackrel{a(M)}{\longrightarrow} s \vdash P^{\prime}$ for some $(M, N) \in(\emptyset ; r)^{\star}$ and $P^{\prime}$. Since $a \in r$, by $\mathcal{Y}^{-}$being an environmental bisimulation and clause 2 of the bisimulation, there is also $t^{\prime} \vdash Q^{\prime}$ such that $t \vdash Q \stackrel{a(N)}{\Longrightarrow} t^{\prime} \vdash Q^{\prime}$, that is, $t \vdash Q \Downarrow_{a}$.

- Case $s \vdash P \downarrow \bar{a}$

We have that $s \vdash P \stackrel{\bar{a}\langle M\rangle}{\longrightarrow} s \vdash P^{\prime}$ for some $M$ and $P^{\prime}$. Since $a \in r$, by $\mathcal{Y}^{-}$ being an environmental bisimulation and clause 3 of the bisimulation, there are also $N, t^{\prime} \vdash Q^{\prime}$ and such that $t \vdash Q \stackrel{\bar{a}\langle N\rangle}{\Longrightarrow} t^{\prime} \vdash Q^{\prime}$, that is, $t \vdash Q \Downarrow_{a}$.

3. Clause Converse of 1,2 on $Q$

Similar to 1, 2.

4. Clause $R$ a process, $f n(R) \cap((s \cup t) \backslash r)=\emptyset$

Let $r^{\prime}=f n(R)$; by appealing to the clause 5 of the bisimulation, since the names in $r^{\prime}$ are either fresh or already in $r$, we have that $\left(s, r^{\prime} \vdash\right.$ $P) \mathcal{Y}_{\emptyset ; r r^{\prime}}^{-}\left(t, r^{\prime} \vdash Q\right)$. Also, $(R, R) \in\left(\emptyset ; r r^{\prime}\right)^{\circ}$ and thus, using the up-to context technique, $\left(s, r^{\prime} \vdash P \mid R\right) \mathcal{Y}_{\emptyset ; r r^{\prime}}^{-}\left(t, r^{\prime} \vdash Q \mid R\right)$ since $\left(s, r^{\prime} \vdash \cdot\right) \mathcal{Y}_{\mathcal{E} ; r r^{\prime}}^{-}\left(t, r^{\prime} \vdash \cdot\right)$ is preserved by parallel composition of processes from $\left(\emptyset ; r r^{\prime}\right)^{\circ}$. Therefore, $\left(r r^{\prime}, s r^{\prime}, P\left|R, t r^{\prime}, Q\right| R\right) \in \mathcal{Z}$.

Conclusion: we showed that $\mathcal{Z} \subseteq \approx$ and as $(f \vdash P) \mathcal{Y}_{\emptyset ; r}^{-}(f \vdash Q)$ for $r \subseteq f=f n(P, Q)$ implies $(r, f, P, f, Q) \in \mathcal{Z}$, we have that $f \vdash P \approx_{r} f \vdash Q$. 


\section{Reduction-closed barbed congruence from environmental bisimulations}

Definition B.44. Capturing reduction-closed barbed congruence $\cong i$ is the largest binary relation on variables configurations indexed by a set of names $r \subseteq s \cap t$ such that when $s \vdash P \cong_{r} t \vdash Q$,

$-s \vdash P \stackrel{\tau}{\rightarrow} s^{\prime} \vdash P^{\prime}$ implies there are $Q^{\prime}$ and $t^{\prime}$ such that $t \vdash Q \Rightarrow t^{\prime} \vdash Q^{\prime}$ and $s^{\prime} \vdash P^{\prime} \cong_{r} t^{\prime} \vdash Q^{\prime}$,

$-s \vdash P \downarrow_{\mu}$ implies $t \vdash Q \Downarrow_{\mu}$, if $\mu \in r$ or $\bar{\mu} \in r$,

- the converse of the above two on $Q$, and

- for all context $C_{p}$ with holes for process and which can capture names such that $f n\left(C_{p}\right) \cap((s \cup t) \backslash r)=\emptyset, b n\left(C_{p}\right) \cap((s \cup t) \backslash r)=\emptyset$, we have $s \cup f n\left(C_{p}\right) \vdash$ $C_{p}[P] \cong_{r, f n\left(C_{p}\right)} t \cup f n\left(C_{p}\right) \vdash C_{p}[Q]$.

Theorem B.45. There exist $P, Q, r$ and a such that $\{r, a\}=f n(P, Q)$ and $(r, a \vdash \bar{a}\langle P\rangle) \mathcal{Y}_{\emptyset ; r a}(r, a \vdash \bar{a}\langle Q\rangle)$ but not $r, a \vdash P \cong_{r a} r, a \vdash Q$.

Proof. We provide such $P, Q, r$

We let $P_{1 x}=a(X) \cdot(X \mid i(Y) \cdot \bar{x}) \mid x . \bar{f}$, let $Q_{1 x}=a(X) \cdot(X \mid i(Y) . Y) \mid x . \bar{f}$, let $P_{2 x}=\bar{a}\langle\bar{i}\langle\bar{x}\rangle\rangle$ and $Q_{2 x}=\bar{a}\langle\bar{i}\langle\bar{x}\rangle\rangle$.

We then consider the two processes

$$
P=\nu i .\left(\bar{b}\left\langle P_{1 m}\right\rangle \mid \bar{c}\left\langle P_{2 m}\right\rangle\right)=\nu i .(\bar{b}\langle a(X) .(X \mid i(Y) \cdot \bar{m}) \mid m . \bar{f}\rangle \mid \bar{a}\langle\bar{i}\langle\bar{m}\rangle\rangle)
$$

and

$$
Q=\nu i .\left(\bar{b}\left\langle Q_{1 m}\right\rangle \mid \bar{c}\left\langle Q_{2 m}\right\rangle\right)=\nu i .(\bar{b}\langle a(X) .(X \mid i(Y) . Y) \mid m \cdot \bar{f}\rangle \mid \bar{a}\langle\bar{i}\langle\bar{m}\rangle\rangle)
$$

which are such that

$$
f n(a, P, Q) \vdash \bar{a}\langle P\rangle \mathcal{Y}_{\emptyset ; f n(a, P, Q)} f n(a, P, Q) \vdash \bar{a}\langle Q\rangle
$$

We let $n=\{a, b, c, f, m\}$ the set of there free names, and compare $P$ and $Q$ under this knowledge:

$$
n \vdash \nu i .\left(\bar{b}\left\langle P_{1 m}\right\rangle \mid \bar{c}\left\langle P_{2 m}\right\rangle\right) \cong_{n} n \vdash \nu i .\left(\bar{b}\left\langle Q_{1 m}\right\rangle \mid \bar{c}\left\langle Q_{2 m}\right\rangle\right)
$$

We first consider a reduction on the left hand-side (lhs) to create the name $i$, and let the right hand-side (rhs) follow weakly. We then do a reduction on the rhs to force the creation of the (other) name $i$ if it had not be done, and let the lhs as is. We then are guaranteed to have:

$$
n, i \vdash \bar{b}\left\langle P_{1 m}\right\rangle\left|\bar{c}\left\langle P_{2 m}\right\rangle \cong_{n} n, i \vdash \bar{b}\left\langle Q_{1 m}\right\rangle\right| \bar{c}\left\langle Q_{2 m}\right\rangle
$$

We can now create a context $C_{p}=\bar{a}\left\langle\nu m .[]_{1}\right\rangle|a(X) .(X \mid X)| g . b . b(X) . c . c(Y) .(X \mid$ $Y$ ) that will allow us to:

- capture the name $m$,

- duplicate the processes with captured names, 
- discard subprocesses and keep others

- have a side of the equivalence always show a barb while the other can never.

$$
n, i, g \vdash C_{p}\left[\bar{b}\left\langle P_{1 m}\right\rangle \mid \bar{c}\left\langle P_{2 m}\right\rangle\right] \cong_{n, g} n, i, g \vdash C_{p}\left[\bar{b}\left\langle Q_{1 m}\right\rangle \mid \bar{c}\left\langle Q_{2 m}\right\rangle\right]
$$

Then, we force the reaction between $\bar{a}$ and $a(X)$ and immediately after we create the names $x$ and $y$. The conditions on the fresh barb $g$ will ensure that we do not have (weak) uncontrolled reactions. We write $C_{p}^{\prime}$ for $g . b . b(X) . c . c(Y) .(X \mid$ $Y$ ) since we lack space.

$n, i, g, x, y \vdash \bar{b}\left\langle P_{1 x}\right\rangle\left|\bar{c}\left\langle P_{2 x}\right\rangle\right| \bar{b}\left\langle P_{1 y}\right\rangle\left|\bar{c}\left\langle P_{2 y}\right\rangle\right| C_{p}^{\prime} \cong_{n, g} n, i, g, x, y \vdash \bar{b}\left\langle Q_{1 x}\right\rangle\left|\bar{c}\left\langle Q_{2 x}\right\rangle\right| \bar{b}\left\langle Q_{1 y}\right\rangle\left|\bar{c}\left\langle Q_{2 y}\right\rangle\right| C_{p}^{\prime}$

We can now spawn in parallel a process $\bar{g}$ to remove the guard of $C_{p}^{\prime}$, and then in the rhs, consider the reactions that select $Q_{1 x}$ and $Q_{2 y}$, discarding the other subprocesses. The left hand-side will follow, choosing to discard two subprocesses, keeping two others which we will call $P_{1}$ (whose "free private name" we will call $z$, whichever of $x$ or $y$ it is) and $P_{2}$. The fact that $P_{1}$ and $P_{2}$ may weakly continue to react after being sent is not an issue, and we will consider they did not.

$$
n, i, g, x, y \vdash P_{1}\left|P_{2} \cong_{n, g} n, i, g, x, y \vdash Q_{1 x}\right| Q_{2 y}
$$

Then, $P_{1}$ and $Q_{1}$ may react on channel $a$, and rhs will follow somehow.

$$
n, i, g, x, y \vdash \bar{i}\langle x \text { or } y\rangle|i(Y) \cdot \bar{z}| z \cdot \bar{f} \cong_{n, g} n, i, g, x, y \vdash \ldots
$$

Then lhs can react on $i$, and both $\bar{z}$ and $z \cdot \bar{f}$ will react, exhibiting barb $\bar{f}$.

$$
n, i, g, x, y \vdash \bar{i}\langle x \text { or } y\rangle|i(Y) . \bar{z}| z \cdot \bar{f} \stackrel{\tau}{\rightarrow} n, i, g, x, y \vdash \bar{x} \mid x \cdot \bar{f} \stackrel{\tau}{\rightarrow} n, i, g, x, y \vdash \bar{f} \downarrow_{\bar{f}}
$$

However, rhs will never be able to exhibit barb $\bar{f}$ since it will be stuck at

$$
n, i, g, x, y \vdash \bar{i}\langle x\rangle|i(Y) . Y| y . \bar{f} \stackrel{\tau}{\rightarrow} n, i, g, x, y \vdash \bar{x} \mid y . \bar{f}
$$

where $x$ and $y$ cannot react.

Lemma B.46. $\left\{(r, s, P, t, Q) \mid r \subseteq r^{\prime},\left(r^{\prime}, s, P, t, Q\right) \in \dot{\approx}\right\} \in \dot{\approx}$.

Proof. By verifying the clauses of $\dot{\approx}$.

Lemma B.47. Let $\mathcal{Y}$ be the environmental bisimilarity up-to context, and

$$
\begin{aligned}
\mathcal{S}=\{(r, s, P, t, Q) \mid & P \leq C\left[\widetilde{P}^{+}\right], Q \leq C\left[\widetilde{Q}^{+}\right], \\
& f n\left(P^{+}\right) \subseteq s_{0}, f n\left(Q^{+}\right) \subseteq t_{0}, \\
& \left(\widetilde{P}^{+}, \widetilde{Q}^{+}\right) \subseteq \mathcal{E}, \\
& r \subseteq r_{0} \cup f n(C), \\
& s=s_{0} \cup f n(C), t=t_{0} \cup f n(C) \\
& f n(C) \cap\left((s \cup t) \backslash r_{0}\right)=\emptyset, \\
& b n(C) \cap(s \cup t)=\emptyset, \\
& \left.\left(s_{0} \vdash 0\right) \mathcal{Y}_{\mathcal{E} ; r_{0}}\left(t_{0} \vdash 0\right)\right\}
\end{aligned}
$$


for contexts $C$ for processes. We show that for all closed $(r, s, P, t, Q) \in \mathcal{S}$, if $s \vdash P \downarrow_{\mu}$ and $\mu$ or $\bar{\mu}$ is in $r$, then $t \vdash Q \Downarrow_{\mu}$, and that if $s \vdash P \rightarrow s^{\prime} \vdash P^{\prime}$ then $t \vdash Q \Rightarrow t^{\prime} \vdash Q^{\prime}$ for some $t^{\prime} \vdash Q^{\prime}$ with $\left(r, s^{\prime}, P^{\prime}, t^{\prime}, Q^{\prime}\right) \in \mathcal{S}$, and conversely.

Proof. By induction on the transition derivation of $s \vdash P \stackrel{\alpha}{\rightarrow} s^{\prime} \vdash P^{\prime}$ with $(r, s, P, t, Q) \in \mathcal{S}$. We prove the two properties separately. In both situations, there is a case analysis on who does the transition: the context's erasure or some $P_{i}$. By symmetricity, we do not show the converse proofs on $Q$ 's transition; they are similar. We write $\operatorname{run}^{*}(P)$ to mean $\operatorname{run}^{\natural}\left(\ldots\left(\operatorname{run}^{\natural}(P)\right) \ldots\right)$, and $C_{p}^{-}, C_{q}^{-}$for two possibly different erasures of the same $C$.

Barbs: the cases necessary to check for barbs are Ho-In, Ho-Out, PAR-R, Par-L, Rep, Transp, and Passiv.

- Ho-IN

- Subcase $C_{p}^{-}[\widetilde{P}]=a(X) \cdot C_{1 p}^{-}[\widetilde{P}]$

If $s \vdash C_{p}^{-}[\widetilde{P}] \downarrow_{a}$, we have $s \vdash a(X) . C_{1 p}^{-}[\widetilde{P}] \stackrel{a(M)}{\longrightarrow} \cdot$. Thus $t \vdash C_{q}^{-}[\widetilde{Q}]=t \vdash$ $\operatorname{run}^{*}\left(a(X) \cdot C_{1 q}^{-}[\widetilde{Q}]\right) \stackrel{\text { run }}{\longrightarrow} t \vdash a(X) . C_{1 q}^{-}[\widetilde{Q}] \stackrel{a(N)}{\longrightarrow} \cdot$, i.e. $C_{q}^{-}[\widetilde{Q}] \Downarrow_{a}$

- Subcase []$_{i}$

By $(\mathcal{E}, r, s, 0, t, 0) \in \mathcal{Y}$, we have $\left(s \vdash P_{i}\right) \mathcal{Y}_{\emptyset ; r}^{-}\left(t \vdash Q_{i}\right)$ up-to environment, hence by Theorem B.44 $t \vdash Q_{i} \Downarrow_{a}$ if $s \vdash P_{i} \downarrow_{a}$, hence $t \vdash r u n^{*} Q_{i} \Downarrow_{a}$, that is $t \vdash C_{p}^{-}[\widetilde{Q}] \Downarrow_{a}$.

- Ho-Out

- Subcase $C_{p}^{-}[\widetilde{P}]=\bar{a}\left\langle D_{p}^{-}[\widetilde{P}]\right\rangle \cdot C_{1 p}^{-}[\widetilde{P}]$

If $s \vdash C_{p}^{-}[\widetilde{P}] \downarrow_{\bar{a}}$, we have $s \vdash \bar{a}\left\langle{ }^{\prime} D_{p}^{-}[\widetilde{P}]\right\rangle \cdot C_{1 p}^{-}[\widetilde{P}] \stackrel{\bar{a}\left\langle{ }^{\prime} D_{p}^{-}[\widetilde{P}]\right\rangle}{\longrightarrow} \cdot$. Thus, $t \vdash$ $C_{q}^{-}[\widetilde{Q}]=t \vdash \operatorname{run} n^{*}\left(\bar{a}\left\langle{ }^{\prime} D_{p}^{-}[\widetilde{Q}]\right\rangle . C_{1 q}^{-}[\widetilde{Q}]\right) \stackrel{\text { run }}{\longrightarrow} t \vdash \bar{a}\left\langle{ }^{\prime} D_{p}^{-}[\widetilde{Q}]\right\rangle . C_{1 q}^{-}[\widetilde{Q}] \stackrel{\bar{a}\left\langle{ }^{\prime} D_{p}^{-}[\widetilde{Q}]\right\rangle}{\longrightarrow}$ - i.e. $t \vdash C_{q}^{-}[\widetilde{Q}] \Downarrow_{\bar{a}}$

- Subcase []$_{i}$

By $(\mathcal{E}, r, s, 0, t, 0) \in \mathcal{Y}$, we have $\left(s \vdash P_{i}\right) \mathcal{Y}_{\emptyset ; r}^{-}\left(t \vdash Q_{i}\right)$, hence $t \vdash Q_{i} \Downarrow_{\bar{a}}$ if $s \vdash P_{i} \downarrow_{\bar{a}}$, hence $t \vdash$ run $^{*} Q_{i} \Downarrow_{\bar{a}}$, that is $t \vdash C_{p}^{-}[\widetilde{Q}] \Downarrow_{\bar{a}}$.

In all the other cases, this subcase is similar, and we will thus not write it anymore.

- PASSIV

$s \vdash C_{p}^{-}[\widetilde{P}]=s \vdash a\left[C_{1 p}^{-}[\widetilde{P}]\right] \downarrow_{\bar{a}}$. Trivially $t \vdash a\left[C_{1 q}^{-}[\widetilde{Q}]\right] \downarrow_{\bar{a}}$, hence $t \vdash C_{q}^{-}[\widetilde{Q}]=$ $t \vdash \operatorname{run}^{*}\left(a\left[C_{1 q}^{-}[\widetilde{Q}]\right]\right) \Downarrow_{\bar{a}}$.

- PAR-L

$s \vdash C_{p}^{-}[\widetilde{P}]=s \vdash C_{1 p}^{-}[\widetilde{P}] \mid C_{2 p}^{-}[\widetilde{P}] \downarrow_{\mu}$ hence $s \vdash C_{1 p}^{-}[\widetilde{P}] \downarrow_{\mu}$. By the induction hypothesis, $t \vdash C_{1 q}^{-}[\widetilde{Q}] \Downarrow_{\mu}$ hence $t \vdash C_{q}^{-}[\widetilde{Q}]=t \vdash \operatorname{run}^{* \bullet}\left(C_{1 q}^{-}[\widetilde{Q}] \mid C_{2 q}^{-}[\widetilde{Q}]\right) \Downarrow_{\mu}$.

- PAR-R

Similarly.

- REP

$s \vdash C_{p}^{-}[\widetilde{P}]=s \vdash ! C_{1 p}^{-}[\widetilde{P}] \downarrow_{\mu}$, hence $s \vdash ! C_{1 p}^{-}[\widetilde{P}] \mid C_{1 p}^{-}[\widetilde{P}] \downarrow_{\mu}$. By the induction hypothesis, $t \vdash ! C_{1 q}^{-}[\widetilde{Q}] \mid C_{1 q}^{-}[\widetilde{Q}] \Downarrow_{\mu}$, hence $t \vdash C_{q}^{-}[\widetilde{Q}]=t \vdash \operatorname{run}^{*}\left(! C_{1 q}^{-}[\widetilde{Q}]\right) \Downarrow_{\mu}$. 


\section{- TRANSP}

$s \vdash C_{p}^{-}[\widetilde{P}]=s \vdash a\left[C_{1 p}^{-}[\widetilde{P}]\right] \downarrow_{\mu}$, hence $s \vdash C_{1 p}^{-}[\widetilde{P}] \downarrow_{\mu}$. By the induction hypothesis, $t \vdash C_{1 q}^{-}[\widetilde{Q}] \Downarrow_{\mu}$, hence $t \vdash a\left[C_{1 q}^{-}[\widetilde{Q}]\right] \Downarrow_{\mu}$, hence $t \vdash C_{q}^{-}[\widetilde{Q}]=t \vdash$ $\operatorname{run}^{*}\left(a\left[C_{1 q}^{-}[\widetilde{Q}]\right]\right) \Downarrow_{\mu}$.

Reductions: the cases necessary to check for reduction closure are RuN, Transp, Par-L, Par-R, Rep, Create, React-L, and React-R.

The CREATE case is particularly different from the others.

- CReate

- $s \vdash C_{p}^{-}[\widetilde{P}]=s \vdash \nu x . C_{1 p}^{-}[\widetilde{P}] \rightarrow s, x \vdash C_{1 p}^{-}[\widetilde{P}]$ and $t \vdash C_{q}^{-}[\widetilde{Q}]=t \vdash$ $\operatorname{run}^{* \bullet}\left(\nu x . C_{1 q}^{-}[\widetilde{Q}]\right)$

A similar transition can be taken by $Q: t \vdash C_{q}^{-}[\widetilde{Q}]=\operatorname{run}^{*}\left(\nu x . C_{1 q}^{-}[\widetilde{Q}]\right) \stackrel{\text { run }}{\Longrightarrow}$ $t \vdash \nu x . C_{1 q}^{-}[\widetilde{Q}] \rightarrow t, x \vdash C_{1 q}^{-}[\widetilde{Q}]$ (we can choose the same name $x$ for both creations).

By $(s \vdash 0) \mathcal{Y}_{\mathcal{E} ; r}^{-}(t \vdash 0)$ and freshness of $x$, we have $(s, x \vdash 0) \mathcal{Y}_{\mathcal{E} ; r x}^{-}(t, x \vdash 0)$ which implies $\left(r, s x, C_{1 p}^{-}[\widetilde{P}], t x, C_{1 q}^{-}[\widetilde{Q}]\right) \in \mathcal{S}$.

- []$_{i}$

We have $\left(s \vdash a\left[P_{i}\right]\right) \mathcal{Y}_{\mathcal{E} ; r}^{-}\left(t \vdash a\left[Q_{i}\right]\right)$ and $s \vdash a\left[P_{i}\right] \rightarrow s, x \vdash a\left[P_{i}^{\prime}\right] \stackrel{\bar{a}\left\langle{ }^{‘} P_{i}^{\prime}\right\rangle}{\longrightarrow}$ $s, x \vdash 0$, so $t \vdash a\left[Q_{i}\right] \Rightarrow t^{\prime \prime} \vdash a\left[Q_{i}^{\prime \prime}\right] \stackrel{\bar{a}\left\langle{ }^{\prime} Q_{i}^{\prime}\right\rangle}{\longrightarrow} t^{\prime} \vdash 0$, and $(s, x \vdash 0) \mathcal{Y}_{\left({ }^{\prime} P_{i}^{\prime},{ }^{\prime} Q_{i}^{\prime}\right) \oplus \mathcal{E} ; r}^{-}$ $\left(t^{\prime} \vdash 0\right)$, hence $\left(r, s x, P_{i}^{\prime}, t^{\prime}, Q_{i}^{\prime}\right) \in \mathcal{S}$. Also, $t \vdash \operatorname{run}^{*}\left({ }^{\prime} Q_{i}\right) \Rightarrow t \vdash Q_{i} \Rightarrow t^{\prime} \vdash Q_{i}^{\prime}$ and we are done.

- RUN

- $s \vdash \operatorname{run}\left({ }^{(} C_{1 p}^{-}[\widetilde{P}]\right) \rightarrow s \vdash C_{1 p}^{-}[\widetilde{P}]$

We have $s \vdash \operatorname{run}\left({ }^{\prime} C_{1 p}^{-}[\widetilde{P}]\right) \rightarrow s \vdash C_{1 p}^{-}[\widetilde{P}]$ and $C_{1 p}^{-}[\widetilde{P}] \leq C\left[\widetilde{P}^{+}\right]$. We still have $C_{q}^{-}[\widetilde{Q}] \leq C\left[\widetilde{Q}^{+}\right]$, so $\left(r, s, P^{\prime}, t, Q\right) \in \mathcal{S}$ and we are done.

- []$_{i}$

We have $\left(s \vdash a\left[P_{i}\right]\right) \mathcal{Y}_{\mathcal{E} ; r}^{-}\left(t \vdash a\left[Q_{i}\right]\right)$ and $s \vdash a\left[P_{i}\right] \rightarrow s \vdash a\left[P_{i}^{\prime}\right] \stackrel{\bar{a}\left\langle^{‘} P_{i}^{\prime}\right\rangle}{\longrightarrow} s \vdash 0$, so $t \vdash a\left[Q_{i}\right] \Rightarrow t^{\prime \prime} \vdash a\left[Q_{i}^{\prime \prime}\right] \stackrel{\bar{a}\left\langle{ }^{\prime} Q_{i}^{\prime}\right\rangle}{\Longrightarrow} t^{\prime} \vdash 0$, and $(s \vdash 0) \mathcal{Y}_{\left({ }^{\prime} P_{i}^{\prime},{ }^{\prime} Q_{i}^{\prime}\right) \oplus \mathcal{E} ; r}^{-}\left(t^{\prime} \vdash 0\right)$, hence $\left(r, s, P_{i}^{\prime}, t^{\prime}, Q_{i}^{\prime}\right) \in \mathcal{S}$. Also, $t \vdash \operatorname{run}^{*}\left({ }^{\prime} Q_{i}\right) \Rightarrow t \vdash Q_{i} \Rightarrow t^{\prime} \vdash Q_{i}^{\prime}$ and we are done.

- TRANSP

- $s \vdash a\left[C_{1 p}^{-}[\widetilde{P}]\right] \rightarrow s^{\prime} \vdash a[R]$

We have $s \vdash C_{1 p}^{-}[\widetilde{P}] \rightarrow s^{\prime} \vdash R$, so, by the induction hypothesis $t \vdash$ $C_{1 q}^{-}[\widetilde{Q}] \Rightarrow t^{\prime} \vdash S$ and $\left(r, s^{\prime}, t^{\prime}, R, S\right) \in \mathcal{S}$. Therefore $t \vdash a\left[C_{1 q}^{-}[\widetilde{Q}]\right] \Rightarrow t^{\prime} \vdash$ $a[S]$, and $\left(r, s^{\prime}, a[R], t^{\prime}, a[S]\right) \in \mathcal{S}$ since the fresh names $\left(t^{\prime} \backslash t\right)$ can be guaranteed different from $a$.

- []$_{i}$

We have $\left(s \vdash a\left[P_{i}\right]\right) \mathcal{Y}_{\mathcal{E} ; r}^{-}\left(t \vdash a\left[Q_{i}\right]\right), s \vdash a\left[P_{i}\right] \rightarrow s^{\prime} \vdash a\left[P_{i}^{\prime}\right] \stackrel{\bar{a}\left\langle{ }^{\prime} P_{i}^{\prime}\right\rangle}{\longrightarrow} s^{\prime} \vdash 0$. So, $t \vdash a\left[Q_{i}\right] \Rightarrow t^{\prime \prime} \vdash a\left[Q_{i}^{\prime \prime}\right] \stackrel{\bar{a}\left\langle{ }^{\prime} Q_{i}^{\prime}\right\rangle}{\longrightarrow} t^{\prime} \vdash 0$, and $\left(s^{\prime} \vdash 0\right) \mathcal{Y}_{\left({ }^{\prime} P_{i}^{\prime},{ }^{\prime} Q_{i}^{\prime}\right) \oplus \mathcal{E} ; r}^{-}\left(t^{\prime} \vdash 0\right)$, 
hence $\left(r, s^{\prime}, a\left[P_{i}^{\prime}\right], t^{\prime}, a\left[Q_{i}^{\prime}\right]\right) \in \mathcal{S}$ since $a$ can be guaranteed different from $\left(s^{\prime} \backslash s\right)$ and $t^{\prime} \backslash t$. Also, $t \vdash \operatorname{run}^{*}\left({ }^{\prime} Q_{i}\right) \Rightarrow t \vdash Q_{i} \Rightarrow t^{\prime} \vdash Q_{i}^{\prime}$ and we are done. Again, this subcase always holds similarly, and thus it is not repeated below.

- PAR-L

$s \vdash C_{p}^{-}[\widetilde{P}]=s \vdash C_{1 p}^{-}[\widetilde{P}]\left|C_{2 p}^{-}[\widetilde{P}] \rightarrow s^{\prime} \vdash R\right| C_{2 p}^{-}[\widetilde{P}]$, i.e. $s \vdash C_{1 p}^{-}[\widetilde{P}] \rightarrow s^{\prime} \vdash R$. So, by the induction hypothesis, $t \vdash C_{1 q}^{-}[\widetilde{Q}] \Rightarrow t^{\prime} \vdash S$ and $\left(r, s^{\prime}, R, t^{\prime}, S\right) \in \mathcal{S}$. Therefore, $t \vdash \operatorname{run}^{*}\left(C_{q}^{-}[\widetilde{P}]\right) \Rightarrow t^{\prime} \vdash S \mid C_{2 q}^{-}[\widetilde{Q}]$, and also $\left(r, s^{\prime}, R\left|C_{2 p}^{-}[\widetilde{P}], t^{\prime}, S\right|\right.$ $\left.C_{2 q}^{-}[\widetilde{Q}]\right) \in \mathcal{S}$.

- PAR-R

Similarly

- REP

$s \vdash C_{p}^{-}[\widetilde{P}]=s \vdash ! C_{1 p}^{-}[\widetilde{P}] \rightarrow s^{\prime} \vdash R$, hence $s \vdash ! C_{1 p}^{-}[\widetilde{P}] \mid C_{1 p}^{-}[\widetilde{P}] \rightarrow s^{\prime} \vdash R$. By the induction hypothesis, $t \vdash ! C_{1 q}^{-}[\widetilde{Q}] \mid C_{1 q}^{-}[\widetilde{Q}] \Rightarrow t^{\prime} \vdash S$ with $\left(r, s^{\prime}, R, t^{\prime}, S\right) \in \mathcal{S}$, hence, $t \vdash ! C_{1 q}^{-}[\widetilde{Q}] \Rightarrow t^{\prime} \vdash S, t \vdash C_{q}^{-}[\widetilde{Q}]=t \vdash \operatorname{run}^{*}\left(! C_{1 q}^{-}[\widetilde{Q}]\right) \Rightarrow t^{\prime} \vdash S$, and still $\left(r, s^{\prime}, R, t^{\prime}, S\right) \in \mathcal{S}$.

- REACT-L

There are several subcases.

- The two contexts react $s \vdash C_{p}^{-}[\widetilde{P}]=s \vdash C_{1 p}^{-}\left[\widetilde{P}_{0}, \widetilde{P}_{1}\right]\left|C_{2 p}^{-}\left[\widetilde{P}_{2}\right] \rightarrow s \vdash C_{1 p}^{\prime-}\left[\widetilde{P}_{0}\right]\right| C_{2 p}^{\prime-}\left[\widetilde{P}_{2}, \widetilde{P}_{1}\right]$. Of course, $C_{1 q}^{-}$can (weakly) do the same reaction, giving $t \vdash C_{q}^{-}[\widetilde{Q}] \Rightarrow t \vdash$ $C_{1 q}^{\prime-}\left[\widetilde{Q}_{0}\right] \mid C_{2 q}^{\prime-}\left[\widetilde{Q}_{2}, \widetilde{Q}_{1}\right]$ and as expected $\left(r, s, C_{1 p}^{\prime-}\left[\widetilde{P}_{0}\right]\left|C_{2 p}^{\prime-}\left[\widetilde{P}_{2}, \widetilde{P}_{1}\right], t, C_{1 q}^{\prime-}\left[\widetilde{Q}_{0}\right]\right|\right.$ $\left.C_{2 q}^{\prime-}\left[\widetilde{Q}_{2}, \widetilde{Q}_{1}\right]\right) \in \mathcal{S}$.

- $C_{1 p}^{-}\left[\widetilde{P}_{1}\right]$ sends, $P_{i}$ in $C_{2 p}^{-}\left[\widetilde{P}_{2}, P_{i}\right]$ receives. $s \vdash C_{p}^{-}[\widetilde{P}]=s \vdash C_{1 p}^{-}\left[\widetilde{P}_{1}\right]\left|C_{2 p}^{-}\left[\widetilde{P}_{2}, P_{i}\right] \rightarrow s \vdash C_{1 p}^{\prime-}\left[\widetilde{P}_{1}\right]\right| C_{2 p}^{-}\left[\widetilde{P}_{2}, P_{i}^{\prime}\right]$. We know that $C_{1 q}^{-}[\widetilde{Q}]$ can weakly do the same output transition on some channel $o$. Also, we have $\left(s \vdash a\left[P_{i}\right]\right) \mathcal{Y}_{\mathcal{E} ; r}^{-}\left(t \vdash a\left[Q_{i}\right]\right)$ and $s \vdash a\left[P_{i}\right] \stackrel{o}{\rightarrow} s \vdash a\left[P_{i}^{\prime}\right] \stackrel{\bar{a}\left\langle^{‘} P_{i}^{\prime}\right\rangle}{\longrightarrow}$ $s \vdash 0$ for the same channel $o \in r$. So, $t \vdash a\left[Q_{i}\right] \stackrel{o}{\Rightarrow} t^{\prime \prime} \vdash a\left[Q_{i}^{\prime \prime}\right] \stackrel{\bar{a}\left\langle^{\prime} Q_{i}^{\prime}\right\rangle}{\Longrightarrow} t^{\prime} \vdash 0$ with $(s \vdash 0) \mathcal{Y}_{\left({ }^{\circ} P_{i}^{\prime},{ }^{\prime} Q_{i}^{\prime}\right) \oplus \mathcal{E} ; r}\left(t^{\prime} \vdash 0\right)$, hence $t \vdash C_{q}^{-}[\widetilde{Q}] \Rightarrow t^{\prime} \vdash C_{1 q}^{\prime-}\left[\widetilde{Q}_{1}\right] \mid$ $C_{2 q}^{\prime-}\left[\widetilde{Q}_{2}, Q_{i}^{\prime}\right]$ since the input can be done by $Q_{i}$ and since $Q_{i}$ is (weakly) in redex position, and $\left(r, s, C_{1 p}^{\prime-}\left[\widetilde{P}_{1}\right]\left|C_{2 p}^{-}\left[\widetilde{P}_{2}, P_{i}^{\prime}\right], t^{\prime}, C_{1 q}^{\prime-}\left[\widetilde{Q}_{1}\right]\right| C_{2 q}^{\prime-}\left[\widetilde{Q}_{2}, Q_{i}^{\prime}\right]\right) \in$ $\mathcal{S}$.

- $C_{2 p}^{-}\left[\widetilde{P}_{2}\right]$ receives, $P_{i}$ in $C_{1 p}^{-}\left[\widetilde{P}_{1}, P_{i}\right]$ sends $s \vdash C_{p}^{-}[\widetilde{P}]=s \vdash C_{1 p}^{-}\left[\widetilde{P}_{1}, P_{i}\right]\left|C_{2 p}^{-}\left[\widetilde{P}_{2}\right] \rightarrow s \vdash C_{1 p}^{-}\left[\widetilde{P}_{1}, P_{i}^{\prime}\right]\right| C_{2 p}^{\prime-}\left[\widetilde{P}_{2}, P_{j}\right]$. We know that $C_{2 q}^{-}\left[\widetilde{Q}_{2}\right]$ can weakly do the same input transition on some channel $o$. Also, we have $s \vdash\left(a\left[P_{i}\right]\right) \mathcal{Y}_{\mathcal{E} ; r}^{-}\left(t \vdash a\left[Q_{i}\right]\right)$ and $s \vdash a\left[P_{i}\right] \stackrel{\left.\bar{o}^{`}{ }^{\prime} P_{j}\right\rangle}{\longrightarrow} s \vdash$ $a\left[P_{i}^{\prime}\right] \stackrel{\bar{a}\left\langle\left\langle^{\prime} P_{i}^{\prime}\right\rangle\right.}{\longrightarrow} s \vdash 0$, so, $t \vdash a\left[Q_{i}\right] \stackrel{\bar{o}\left\langle{ }^{\prime} Q_{j}\right\rangle}{\longrightarrow} t^{\prime \prime} \vdash a\left[Q_{i}^{\prime \prime}\right] \stackrel{\bar{a}\left\langle{ }^{\prime} Q_{i}^{\prime}\right\rangle}{\longrightarrow} t^{\prime} \vdash 0$ with $(s \vdash 0) \mathcal{Y}_{\left({ }^{\prime} P_{j},{ }^{\prime} Q_{j}\right) \oplus \mathcal{E} ; r}\left(t^{\prime} \vdash 0\right)$, so $t \vdash C_{q}^{-}[\widetilde{Q}] \Rightarrow t^{\prime} \vdash C_{1 q}^{\prime-}\left[\widetilde{Q}_{1}, Q_{i}^{\prime}\right] \mid C_{2 q}^{\prime-}\left[\widetilde{Q}_{2}, Q_{j}\right]$, and $\left(r, s, C_{1 p}^{-}\left[\widetilde{P}_{1}, P_{i}^{\prime}\right]\left|C_{2 p}^{\prime-}\left[\widetilde{P}_{2}, P_{j}\right], t^{\prime}, C_{1 q}^{\prime-}\left[\widetilde{Q}_{1}, Q_{i}^{\prime}\right]\right| C_{2 q}^{\prime-}\left[\widetilde{Q}_{2}, Q_{j}\right]\right) \in \mathcal{S}$. 
- $P_{i}$ in $C_{1 p}^{-}\left[\widetilde{P}_{1}, P_{i}\right]$ and $P_{j}$ in $C_{2 p}^{-}\left[\widetilde{P}_{2}, P_{j}\right]$ react $s \vdash C_{p}^{-}[\widetilde{P}]=s \vdash C_{1 p}^{-}\left[\widetilde{P}_{1}, P_{i}\right]\left|C_{2 p}^{-}\left[\widetilde{P}_{2}, P_{j}\right] \rightarrow s \vdash C_{1 p}^{-}\left[\widetilde{P}_{1}, P_{i}^{\prime}\right]\right| C_{2 p}^{-}\left[\widetilde{P}_{2}, P_{j}^{\prime}\right]$. We have $\left(s, a, b \vdash a\left[P_{i}\right] \mid b\left[P_{j}\right]\right) \mathcal{Y}_{\mathcal{E} ; r a b}^{-}\left(t, a, b \vdash a\left[Q_{i}\right] \mid b\left[Q_{j}\right]\right)$ and $s, a, b \vdash$ $a\left[P_{i}\right]\left|b\left[P_{j}\right] \rightarrow s, a, b \vdash a\left[P_{i}^{\prime}\right]\right| b\left[P_{j}^{\prime}\right] \stackrel{\bar{a}\left\langle{ }^{\prime} P_{i}^{\prime}\right\rangle}{\longrightarrow} \stackrel{\bar{b}\left\langle{ }^{\prime} P_{j}^{\prime}\right\rangle}{\longrightarrow} s, a, b \vdash 0$, so $t, a, b \vdash$ $a\left[Q_{i}\right]\left|b\left[Q_{j}\right] \Rightarrow t^{\prime \prime}, a, b \vdash a\left[Q_{i}^{\prime \prime}\right]\right| b\left[Q_{j}^{\prime \prime}\right] \stackrel{\bar{a}\left\langle\left\langle^{\prime} Q_{i}^{\prime}\right\rangle\right.}{\Longrightarrow} \stackrel{\bar{b}\left\langle{ }^{\prime} Q_{j}^{\prime}\right\rangle}{\Longrightarrow} t^{\prime}, a, b \vdash 0$ with $(s, a, b \vdash$ $0) \mathcal{Y}_{\left({ }^{\prime} P_{j}^{\prime},{ }^{\prime} Q_{j}^{\prime}\right) \oplus\left({ }^{\prime} P_{i}^{\prime},{ }^{\prime} Q_{i}^{\prime}\right) \oplus \mathcal{E} ; r a b}\left(t^{\prime}, a, b \vdash 0\right)$, hence $(s \vdash 0) \mathcal{Y}_{\left({ }^{\circ} P_{j}^{\prime},{ }^{\prime} Q_{j}^{\prime}\right) \oplus\left({ }^{\prime} P_{i}^{\prime},{ }^{\prime} Q_{i}^{\prime}\right) \oplus \mathcal{E} ; r}$ $\left(t^{\prime} \vdash 0\right)$ up-to name creation. Therefore, $t \vdash C_{q}^{-}[\widetilde{Q}] \Rightarrow t^{\prime} \vdash C_{1 q}^{\prime-}\left[\widetilde{Q}_{1}, Q_{i}^{\prime}\right] \mid$ $C_{2 q}^{\prime-}\left[\widetilde{Q}_{2}, Q_{j}^{\prime}\right]$, and $\left(C_{1 p}^{-}\left[\widetilde{P}_{1}, P_{i}^{\prime}\right]\left|C_{2 p}^{-}\left[\widetilde{P}_{2}, P_{j}^{\prime}\right], t^{\prime}, C_{1 q}^{\prime-}\left[\widetilde{Q}_{1}, Q_{i}^{\prime}\right]\right| C_{2 q}^{\prime-}\left[\widetilde{Q}_{2}, Q_{j}^{\prime}\right]\right) \in$

- REACT-R

Similarly

Corollary B.48. For all $P, Q, r$ and a such that $r \subseteq f=f n(P, Q), a \notin f \backslash r$, and $(r, a \vdash \bar{a}\langle P\rangle) \mathcal{Y}_{\emptyset ; r a}(r, a \vdash \bar{a}\langle Q\rangle)$, we have $f, a \vdash P \dot{\sim}_{r a} r, a \vdash Q$.

Proof. By $(f, a \vdash \bar{a}\langle P\rangle) \mathcal{Y}_{\emptyset ; r a}(f, a \vdash \bar{a}\langle Q\rangle)$, we have $(f, a \vdash 0) \mathcal{Y}_{\left\{\left({ }^{\prime} P,{ }^{\prime} Q\right)\right\} ; r a}^{\star}(f, a \vdash$ $0)$, hence $(r, s, P, t, Q) \in \mathcal{S}$ hence $f, a \vdash P \dot{\sim}_{r, a} f, a \vdash Q$ by Lemma B.47. 


\section{Completeness of environmental bisimulation}

Lemma B.49. Run erasure of $\approx$.

Proof. We show that $\left\{(r, s, P, t, Q) \mid P \leq P^{+}, Q \leq Q^{+}, s \vdash P^{+} \approx_{r} t \vdash Q^{+}\right\} \subseteq$ $\approx$.

- Derived from Corollary B.23.

- Derived from Corollary B.23.

- Conversely.

- Let $u=f n(R) \backslash r$. We have $s, u \vdash P^{+}\left|R \approx_{r, u} t, u \vdash Q^{+}\right| R$ by definition of $\approx$ and $P\left|R \leq P^{+}\right| R$ as well as $Q\left|R \leq Q^{+}\right| R$ by definition of $\leq$.

Lemma B.50. [Completeness and environmental bisimulation]

Consider the set

$$
\begin{aligned}
\mathcal{X}=\left\{\left(\mathcal{E}_{x},\right.\right. & \left.r_{x}, s_{x}, P\left|a_{1}\left[P_{1}\right]\right| \ldots\left|a_{n}\left[P_{n}\right], t_{x}, Q\right| a_{1}\left[Q_{1}\right]|\ldots| a_{n}\left[Q_{n}\right]\right) \mid \\
& \left(\left({ }^{\prime} P_{1}, \ldots,{ }^{\prime} P_{n}\right),\left({ }^{\prime} Q_{1}, \ldots,{ }^{\prime} Q_{n}\right)\right) \subseteq \mathcal{E}_{x}, \\
& \left(\widetilde{C}_{i}, \widetilde{Q}_{i}\right)=\mathcal{E} \\
& \mathcal{E}_{x} \subseteq \mathcal{E} \\
& s \vdash P\left|\prod_{i=1}^{n} ! \overline{f_{i}}\left\langle{ }^{\prime} P_{i}\right\rangle \approx_{r} t \vdash Q\right| \prod_{i=1}^{n} ! \overline{f_{i}}\left\langle{ }^{\prime} Q_{i}\right\rangle, \\
& \widetilde{a}_{i} \subseteq r_{x} \\
& r=r_{x} \oplus \widetilde{f}_{i} \oplus e_{x},(\oplus \text { meaning no overlap }) \\
& s=s_{x} \oplus \widetilde{f}_{i} \oplus e_{x}, \\
& t=t_{x} \oplus \widetilde{f}_{i} \oplus e_{x}, \\
& \left.r_{x} \subseteq\left(s_{x} \cap t_{x}\right)\right\} .
\end{aligned}
$$

We show that $\mathcal{X}$ is an environmental bisimulation (up-to $\equiv$ ).

Proof. We check each clause of environmental bisimulation (up-to context) against $\mathcal{X}$. We may just write $\prod$ for both $\prod_{i=1}^{n} ! \overline{f_{i}}\left\langle{ }^{\prime} P_{i}\right\rangle$ and $\prod_{i=1}^{n} ! \bar{f}_{i}\left\langle{ }^{\prime} Q_{i}\right\rangle$, and $\prod_{n+1}$ when there is an element added to the product. Context makes clear what is considered.

- Reduction

1. $s_{x} \vdash P\left|a_{1}\left[P_{1}\right]\right| \ldots\left|a_{n}\left[P_{n}\right] \stackrel{\tau}{\rightarrow} s_{x}^{\prime} \vdash P^{\prime}\right| a_{1}\left[P_{1}\right]|\ldots| a_{n}\left[P_{n}\right]$.

We have $s \vdash P\left|\prod \approx_{r} t \vdash Q\right| \prod$ and $s \vdash P\left|\prod \stackrel{\tau}{\rightarrow} s^{\prime} \vdash P^{\prime}\right| \prod$ with $s_{x}^{\prime}=s_{x} \cup\left(s^{\prime} \backslash s\right)$.

So, by clause 1 of Definition B.5, for some $t^{\prime}$ and $Q^{\prime}$, we have $t \vdash Q \mid$ $\prod \stackrel{\tau}{\Rightarrow} t^{\prime} \vdash Q^{\prime} \mid \prod$ and $s^{\prime} \vdash P^{\prime}\left|\prod \approx_{r} t^{\prime} \vdash Q^{\prime}\right| \prod$ since the other subprocesses in $\prod$ are guarded by a "fresh" guard $f_{i}$ and thus cannot reduce nor react with anything.

Therefore, $t \vdash Q \stackrel{\tau}{\Rightarrow} t^{\prime} \vdash Q^{\prime}$, hence $\left.t_{x} \vdash Q\left|a_{1}\left[Q_{1}\right]\right| \ldots \mid a_{n}\left[Q_{n}\right]\right) \stackrel{\tau}{\Rightarrow} t_{x}^{\prime} \vdash$ $Q^{\prime}\left|a_{1}\left[Q_{1}\right]\right| \ldots \mid a_{n}\left[Q_{n}\right]$ for $t_{x}^{\prime}=t_{x} \cup\left(t^{\prime} \backslash t\right)$. Indeed, since created names could not be free in $\mathcal{E}$ by definition of the LTS (PAR-L, PAR-R), they are not free in $\mathcal{E}_{x}$, and by $r_{x} \subseteq\left(s_{x} \cap t_{x}\right) \subseteq(s \cap t)$ we know that they will not clash with $r_{x}$ either. We will henceforth assume this implicitely. Finally, $\left(\mathcal{E}_{x}, r_{x}, s_{x}^{\prime}, P\left|a_{1}\left[P_{1}\right]\right| \ldots\left|a_{n}\left[P_{n}\right], t_{x}^{\prime}, Q^{\prime}\right| a_{1}\left[Q_{1}\right]|\ldots| a_{n}\left[Q_{n}\right]\right) \in \mathcal{X}$. 
2. $P_{i} \stackrel{\tau}{\rightarrow} P_{i}^{\prime}$.

$s \vdash P\left|\prod \approx_{r} t \vdash Q\right| \prod$ so, for some fresh $g$ and for $r^{\prime}=r+g$, by clause 4 of Definition B.5 we have $s, g \vdash P\left|\prod\right| f_{i}(X) . g\left[\right.$ runX] $\approx_{r^{\prime}} t, g \vdash$ $Q\left|\prod\right| f_{i}(X) . g[$ runX].

Then, we can have a reaction between a copy of $f_{i}\left\langle{ }^{\prime} P_{i}\right\rangle$ drawn from $\prod$, and $f_{i}(X)$, giving $s, g \vdash P\left|\prod\right| f_{i}(X) . g\left[\right.$ runX $\stackrel{\tau}{\rightarrow}^{\rightarrow} s, g \vdash P\left|\prod\right| g\left[\right.$ run $\left.^{\prime} P_{i}\right]$. By clause 1 of Definition B.5, we thus have $t_{a}, Q_{a}$ and $Q_{i a}$ such that, by clause 2 of Definition B.5 and the fact that there is no input barb on unique $f_{i}$ in LHS as well as the fact that there is an output barb on unique $g$, we have $t, g \vdash Q\left|\prod\right| f_{i}(X) . g[$ runX $] \stackrel{\tau}{\Rightarrow} t_{a}, g \vdash Q_{a}\left|\prod\right| g\left[Q_{i a}\right]$ and $s, g \vdash P\left|\prod\right| g\left[\right.$ run $\left.^{6} P_{i}\right] \approx_{r^{\prime}} t_{a}, g \vdash Q_{a}\left|\prod\right| g\left[Q_{i a}\right]$.

LHS can now do a run-transition $s, g \vdash P\left|\prod\right| g\left[\text { run }^{6} P_{i}\right]^{\tau} \stackrel{\tau}{\rightarrow} s, g \vdash P\left|\prod\right| g\left[P_{i}\right]$ and thus, by clause 1 of Definition B.5, we have $t_{b}, Q_{b}$ and $Q_{i b}$ such that $t_{a}, g \vdash Q_{a}\left|\prod\right| g\left[Q_{i a}\right] \stackrel{\tau}{\Rightarrow} t_{b}, g \vdash Q_{b}\left|\prod\right| g\left[Q_{i b}\right]$ and $s, g \vdash P\left|\prod\right| g\left[P_{i}\right] \approx_{r^{\prime}}$ $t_{b}, g \vdash Q_{b}\left|\prod\right| g\left[Q_{i b}\right]$

Then, we can now do the transition that corresponds to $P_{i} \stackrel{\tau}{\rightarrow} P_{i}^{\prime}: s, g \vdash$ $P\left|\prod\right| g\left[P_{i}\right] \stackrel{\tau}{\rightarrow} s^{\prime}, g \vdash P\left|\prod\right| g\left[P_{i}^{\prime}\right]$ and by clause 1 of Definition B.5 (and 2 on barb g), we have $t_{b}, g \vdash Q b\left|\prod\right| g\left[Q_{i b}\right] \stackrel{\tau}{\Rightarrow} t_{c}, g \vdash Q c\left|\prod\right| g\left[Q_{i c}\right]$ and $s^{\prime}, g \vdash P\left|\prod\right| g\left[P_{i}^{\prime}\right] \approx_{r^{\prime}} t_{c}, g \vdash Q c\left|\prod\right| g\left[Q_{i c}\right]$.

Now, we can use clause 4 of Definition B.5 with known name $g$ and fresh name $f_{n+1}$ and have, for $e_{x}=g+f_{n+1}$ and $r^{\prime \prime}=r+e_{x}, s^{\prime}, e_{x} \vdash$ $P\left|\prod\right| g\left[P_{i}^{\prime}\right]\left|g(X) . ! \overline{f_{n+1}}\langle X\rangle \approx_{r^{\prime \prime}} t_{c}, e_{x} \vdash Q_{c}\right| \prod\left|g\left[Q_{i c}\right]\right| g(X) ! ! \overline{f_{n+1}}\langle X\rangle$. We can have a reaction between $g[]$ and $g(): s^{\prime}, e_{x} \vdash P\left|\prod\right| g\left[P_{i}^{\prime}\right] \mid$ $g(X) ! \overline{f_{n+1}}\langle X\rangle \stackrel{\tau}{\rightarrow} s^{\prime}, e_{x} \vdash P\left|\prod\right| ! \overline{f_{n+1}}\left\langle{ }^{\prime} P_{i}^{\prime}\right\rangle$, hence by clause 1 of Definition B.5, as well as clause 3 on barbs $g$ and clause 2 on barb $f_{n+1}$, we have $t_{c}, e_{x} \vdash Q_{c}\left|\prod\right| g\left[Q_{i c}\right]\left|g(X) . ! \overline{f_{n+1}}\langle X\rangle \stackrel{\tau}{\Rightarrow} t^{\prime}, e_{x} \vdash Q^{\prime}\right| \prod \mid ! \overline{f_{n+1}}\left\langle{ }^{\prime} Q_{i d}\right\rangle$ with $Q_{i d}=Q_{i}^{\prime}$ or $\operatorname{run}^{\prime} Q_{i}$, as well as $s^{\prime}, e_{x} \vdash P\left|\prod\right| ! \overline{f_{n+1}}\left\langle{ }^{\prime} P_{i}^{\prime}\right\rangle \approx_{r^{\prime \prime}} t^{\prime}, e_{x} \vdash$ $Q^{\prime}\left|\prod\right| ! \overline{f_{n+1}}\left\langle{ }^{\prime} Q_{i d}\right\rangle$.

If $Q_{i d}=Q_{i}^{\prime}$, then we have $s^{\prime}, e_{x} \vdash P\left|\prod_{n+1} \approx_{r^{\prime \prime}} t^{\prime}, e_{x} \vdash Q^{\prime}\right| \prod_{n+1}$. If $Q_{i d}=\operatorname{run}^{\prime} Q_{i}$, then $Q_{i}$ was not necessary in the reduction, and we have $s^{\prime}, e_{x} \vdash P\left|\prod_{n+1} \approx_{r^{\prime \prime}} t^{\prime}, e_{x} \vdash Q^{\prime}\right| \prod_{n+1}$ by run-erasure of $\approx$, only turning $Q_{i d}=\operatorname{run}^{6} Q_{i}$ into $Q_{i}$. This does not affect the transitions done by $Q$.

As a result from all these transitions notwithstanding the run-erasure, we have that $t_{x} \vdash Q\left|a_{1}\left[Q_{1}\right]\right| \ldots\left|a_{i}\left[Q_{i}\right]\right| \ldots\left|a_{n}\left[Q_{n}\right] \stackrel{\sim}{\Rightarrow} t_{x}^{\prime} \vdash Q^{\prime}\right| a_{1}\left[Q_{1}\right] \mid$ $\ldots\left|a_{i}\left[Q_{i}^{\prime}\right]\right| \ldots \mid a_{n}\left[Q_{n}\right]$ and also $\left(\mathcal{E}_{x}, r_{x}, s_{x}^{\prime}, P\left|a_{1}\left[P_{1}\right]\right| \ldots\left|a_{i}\left[P_{i}^{\prime}\right]\right| \ldots \mid\right.$ $\left.a_{n}\left[P_{n}\right], t_{x}^{\prime}, Q^{\prime}\left|a_{1}\left[Q_{1}\right]\right| \ldots\left|a_{i}\left[Q_{i}^{\prime}\right]\right| \ldots \mid a_{n}\left[Q_{n}\right]\right) \in \mathcal{X}$.

3. $P$ and $P_{i}$ react.

Similar to the above case, but with $s$ instead of $s^{\prime}$ and $s_{x}$ instead of $s_{x}^{\prime}$ (since no name is created by the reaction) and $P^{\prime}$ instead of $P$ after the reaction.

As a result from all these transitions, we have that $t_{x} \vdash Q\left|a_{1}\left[Q_{1}\right]\right| \ldots \mid$ $a_{i}\left[Q_{i}\right]|\ldots| a_{n}\left[Q_{n}\right] \stackrel{\tau}{\Rightarrow} t_{x}^{\prime} \vdash Q^{\prime}\left|a_{1}\left[Q_{1}\right]\right| \ldots\left|a_{i}\left[Q_{i}^{\prime}\right]\right| \ldots \mid a_{n}\left[Q_{n}\right]$ and also $\left(\mathcal{E}_{x}, r_{x}, s_{x}, P^{\prime}\left|a_{1}\left[P_{1}\right]\right| \ldots\left|a_{i}\left[P_{i}^{\prime}\right]\right| \ldots\left|a_{n}\left[P_{n}\right], t_{x}^{\prime}, Q^{\prime}\right| a_{1}\left[Q_{1}\right]|\ldots| a_{i}\left[Q_{i}^{\prime}\right] \mid\right.$ $\left.\ldots \mid a_{n}\left[Q_{n}\right]\right) \in \mathcal{X}$

4. $P_{i}$ and $P_{j}$ react. 
$s \vdash P\left|\prod \approx_{r} t \vdash Q\right| \prod$ so, for some fresh $g$ and $h, e_{x}=g+h$ and $r^{\prime}=r+e_{x}$, using clause 4 of Definition B.5, we have $s, e_{x} \vdash P\left|\prod\right| f_{i}(X) \cdot g[\operatorname{run} X] \mid$ $f_{j}(X) . h[\operatorname{run} X] \approx_{r^{\prime}} t, e_{x} \vdash Q\left|\prod\right| f_{i}(X) \cdot g[\operatorname{run} X] \mid f_{j}(X) \cdot h[$ runX $X$.

We can have a reaction between (a copy of) $! f_{i}\langle\rangle$ and $f_{i}\left(\right.$ ) $: s, e_{x} \vdash P \mid$ $\prod \mid f_{i}(X) . g[$ runX $X] \mid f_{j}(X) . h[$ runX $] \stackrel{\tau}{\rightarrow} s, e_{x} \vdash P\left|\prod\right| g\left[\right.$ run $\left.^{\bullet} P_{i}\right] \mid f_{j}(X) . h[$ runX $]$. By clause 1 of Definition B.5, RHS will do a reduction too, and by clause 3 of Definition B.5 on $f_{i}, g$ and 2 on $f_{j}, ! \overline{f_{i}}\langle\rangle$ and $f_{i}()$ will react too in RHS, while $! \overline{f_{j}}\langle\rangle$ and $f_{j}()$ will stay. In other words: $t, e_{x} \vdash Q\left|\prod\right| f_{i}(X) . g[$ runX $] \mid f_{j}(X) . h[$ runX $] \stackrel{\tau}{\Rightarrow} t_{a}, e_{x} \vdash Q_{a}\left|\prod\right| g\left[Q_{i a}\right] \mid$ $f_{j}(X) . h[$ runX $X]$ with $s, e_{x} \vdash P\left|\prod\right| g\left[\right.$ run $\left.^{\prime} P_{i}\right] \mid f_{j}(X) . h[$ runX $] \approx_{r^{\prime}} t_{a}, e_{x} \vdash$ $Q_{a}\left|\prod\right| g\left[Q_{i a}\right] \mid f_{j}(X) . h[\operatorname{run} X]$.

Similarly, with the reaction $s, e_{x} \vdash P\left|\prod\right| g\left[\right.$ run $\left.^{\prime} P_{i}\right] \mid f_{j}(X) \cdot h[$ runX $] \stackrel{\tau}{\rightarrow}$ $s, e_{x} \vdash P\left|\prod\right| g\left[P_{i}\right] \mid h\left[\right.$ run $\left.^{\prime} P_{j}\right]$ between $! f_{j}\langle\rangle$ and $f_{j}()$, we have $t_{a}, e_{x} \vdash$ $Q_{a}\left|\prod\right| g\left[Q_{i a}\right] \mid f_{j}(X) \cdot h[$ runX $] \stackrel{\tau}{\Rightarrow} t_{b}, e_{x} \vdash Q_{b}\left|\prod\right| g\left[Q_{i b}\right] \mid h\left[Q_{j b}\right]$ with $s, e_{x} \vdash P\left|\prod\right| g\left[\right.$ run $\left.^{\prime} P_{i}\right] \mid h\left[\right.$ run $\left.^{\prime} P_{j}\right] \approx_{r^{\prime}} t_{b}, e_{x} \vdash Q_{b}\left|\prod\right| g\left[Q_{i b}\right] \mid h\left[Q_{j b}\right]$.

Then, we can do 2 run transitions which will be weakly followed by RHS according to clause 1 of Definition B.5, while preserving $g[]$ and $h[$ ] by their uniqueness and clause 3 of Definition B.5, and we obtain $s, e_{x} \vdash P\left|\prod\right| g\left[\right.$ run $\left.^{\natural} P_{i}\right] \mid h\left[\right.$ run $\left.^{\natural} P_{j}\right] \stackrel{\text { run }}{\longrightarrow} \stackrel{\text { run }}{\longrightarrow} \stackrel{\tau}{\longrightarrow} s, e_{x} \vdash P\left|\prod\right| g\left[P_{i}\right] \mid h\left[P_{j}\right]$, $t_{b}, e_{x} \vdash Q_{b}\left|\prod\right| g\left[Q_{i b}\right]\left|h\left[Q_{j b}\right] \stackrel{\tau}{\Rightarrow} t_{c}, e_{x} \vdash Q_{c}\right| \prod\left|g\left[Q_{i c}\right]\right| h\left[Q_{j c}\right]$ and $s, e_{x} \vdash$ $P\left|\prod\right| g\left[P_{i}\right]\left|h\left[P_{j}\right] \approx_{r^{\prime}} t_{c}, e_{x} \vdash Q_{c}\right| \prod\left|g\left[Q_{i c}\right]\right| h\left[Q_{j c}\right]$.

It is now possible to mimick the reaction of $P_{i}$ and $P_{j}$ in $\mathcal{X}: s, e_{x} \vdash$ $P\left|\prod\right| g\left[P_{i}\right]\left|h\left[P_{j}\right] \stackrel{\tau}{\rightarrow} s, e_{x} \vdash P\right| \prod\left|g\left[P_{i}^{\prime}\right]\right| h\left[P_{j}^{\prime}\right]$ which is matched by $t_{c}, e_{x} \vdash Q_{c}\left|\prod\right| g\left[Q_{i c}\right]\left|h\left[Q_{j c}\right] \stackrel{\sim}{\Rightarrow} t_{d}, e_{x} \vdash Q_{d}\right| \prod\left|g\left[Q_{i d}\right]\right| h\left[Q_{j d}\right]$ such that $s, e_{x} \vdash P\left|\prod\right| g\left[P_{i}^{\prime}\right]\left|h\left[P_{j}^{\prime}\right] \approx_{r^{\prime}} t_{d}, e_{x} \vdash Q_{d}\right| \prod\left|g\left[Q_{i d}\right]\right| h\left[Q_{j d}\right]$.

Using clause 4 of Definition B.5, we can now spawn another process with fresh names $f_{n+1}$ and $f_{n+2}$ and have, for $e_{x}^{\prime}=e_{x}+f_{n+1}+f_{n+2}$ and $r^{\prime \prime}=r+e_{x}^{\prime}: s, e_{x}^{\prime} \vdash P\left|\prod\right| g\left[P_{i}^{\prime}\right]\left|h\left[P_{j}^{\prime}\right]\right| g(X) \cdot ! \overline{f_{n+1}}\langle X\rangle \mid h(X) . ! \bar{f}_{n+2}\langle X\rangle \approx_{r^{\prime \prime}}$ $t_{d}, e_{x}^{\prime} \vdash Q_{d}\left|\prod\right| g\left[Q_{i d}\right]\left|h\left[Q_{j d}\right]\right| g(X) \cdot ! \overline{f_{n+1}}\langle X\rangle \mid h(X) . ! \overline{f_{n+2}}\langle X\rangle$.

We can now do a reaction between $g[]$ and $g()$, which will be followed (by clause 1), consuming $g[]$ and $g($ )too but leaving $h[]$ and $h($ ) (by clause 3 and uniqueness of each barb on $g$ and $h$ ), giving $s, e_{x}^{\prime} \vdash P\left|\prod\right| g\left[P_{i}^{\prime}\right] \mid$ $h\left[P_{j}^{\prime}\right]\left|g(X) . ! \overline{f_{n+1}}\langle X\rangle\right| h(X) ! \bar{f}_{n+2}\langle X\rangle \stackrel{\tau}{\rightarrow} s, e_{x}^{\prime} \vdash P\left|\prod\right| h\left[P_{j}^{\prime}\right]\left|! \overline{f_{n+1}}\left\langle{ }^{\prime} P_{i}^{\prime}\right\rangle\right|$ $h(X) . ! \overline{f_{n+2}}\langle X\rangle$ as well as $t_{d}, e_{x}^{\prime} \vdash Q_{d}\left|\prod\right| g\left[Q_{i d}\right]\left|h\left[Q_{j d}\right]\right| g(X) . ! \overline{f_{n+1}}\langle X\rangle \mid$ $h(X) . ! \overline{f_{n+2}}\langle X\rangle \stackrel{\tau}{\Rightarrow} t_{e}, e_{x}^{\prime} \vdash Q e\left|\prod\right| h\left[Q_{i e}\right]\left|! \overline{f_{n+1}}\left\langle{ }^{\prime} Q_{i e}\right\rangle\right| h(X) . ! \overline{f_{n+2}}\langle X\rangle$ and $s, e_{x}^{\prime} \vdash P\left|\prod\right| h\left[P_{j}^{\prime}\right]\left|! \overline{f_{n+1}}\left\langle{ }^{\prime} P_{i}^{\prime}\right\rangle\right| h(X) . ! \overline{f_{n+2}}\langle X\rangle \approx_{r^{\prime \prime}} t_{e}, e_{x}^{\prime} \vdash Q e\left|\prod\right| h\left[Q_{i e}\right] \mid$ $! \overline{f_{n+1}}\left\langle\cdot Q_{i e}\right\rangle \mid h(X) . ! \overline{f_{n+2}}\langle X\rangle$.

Similarly, we can have and follow a reaction between $! h[]$ and $h()$ and have $s, e_{x}^{\prime} \vdash P\left|\prod\right| ! \overline{f_{n+1}}\left\langle{ }^{\prime} P_{i}^{\prime}\right\rangle\left|\overline{f_{n+2}}\left\langle{ }^{\prime} P_{j}^{\prime}\right\rangle \approx_{r^{\prime \prime}} t^{\prime}, e_{x}^{\prime} \vdash Q^{\prime}\right| \prod\left|! \overline{f_{n+1}}\left\langle{ }^{\prime} Q_{i e}\right\rangle\right|$ $\overline{f_{n+2}}\left\langle{ }^{\prime} Q_{i e}\right\rangle$.

As in the previous cases, we have that $Q_{i e}$ (resp. $Q_{i e}$ ) is either $\operatorname{run}^{\prime} Q_{i}$ or $Q_{i}^{\prime}$ (resp. $\operatorname{run}^{\prime} Q_{j}$ or $Q_{j}^{\prime}$ ) and we can use run-erasure if necessary without interfering with the transitions. 
As a result from all these transitions, we have that $t_{x} \vdash Q\left|a_{1}\left[Q_{1}\right]\right| \ldots \mid$ $a_{i}\left[Q_{i}\right]\left|a_{j}\left[Q_{j}\right]\right| \ldots\left|a_{n}\left[Q_{n}\right] \stackrel{\tau}{\Rightarrow} t_{x}^{\prime} \vdash Q^{\prime}\right| a_{1}\left[Q_{1}\right]|\ldots| a_{i}\left[Q_{i}^{\prime}\right]\left|a_{j}\left[Q_{j}^{\prime}\right]\right| \ldots \mid a_{n}\left[Q_{n}\right]$ and also $\left(\mathcal{E}_{x}, r_{x}, s_{x}, P\left|a_{1}\left[P_{1}\right]\right| \ldots\left|a_{i}\left[P_{i}^{\prime}\right]\right| a_{j}\left[P_{j}^{\prime}\right]|\ldots| a_{n}\left[P_{n}\right], t_{x}^{\prime}, Q^{\prime}\left|a_{1}\left[Q_{1}\right]\right|\right.$ $\left.\ldots\left|a_{i}\left[Q_{i}^{\prime}\right]\right| a_{j}\left[Q_{j}^{\prime}\right]|\ldots| a_{n}\left[Q_{n}\right]\right) \in \mathcal{X}$

5. $P$ passivates $a\left[P_{i}\right]$.

$s \vdash P\left|\prod \approx_{r} t \vdash Q\right| \prod$ so, for some fresh $d$, and by clause 4 of Definition B.5 $s, d \vdash P\left|\prod\right| f_{i}(X) . ! \bar{a}\langle X\rangle . d \approx_{r+d} t, d \vdash Q\left|\prod\right| f_{i}(X) . ! \bar{a}\langle X\rangle . d$.

Then, we can have a reaction between (a copy of) ! $f_{i}\langle\rangle$ and $f_{i}(): s, d \vdash$ $P\left|\prod\right| f_{i}(X) . ! \bar{a}\langle X\rangle . d \stackrel{\tau}{\rightarrow} s, d \vdash P\left|\prod\right| ! \bar{a}\left\langle{ }^{\prime} P_{i}\right\rangle . d$. By clause 1 of Definition B.5, RHS can weakly follow: $t, d \vdash Q\left|\prod\right| f_{i}(X) . ! \bar{a}\langle X\rangle . d \stackrel{\tau}{\Rightarrow} t_{a}, d \vdash Q_{a}\left|\prod\right| R_{a}$ for some $R_{a}$ (which can only be one of $! \bar{a}\left\langle{ }^{\prime} Q_{i}\right\rangle . d$, or $d$ (since $d$ is fresh and since clause 3 enforces we consumed $\left.f_{i}(X)\right)$ ) and we have $s, d \vdash$ $P\left|\prod\right| ! \bar{a}\langle X\rangle . d \approx_{r+d} t_{a}, d \vdash Q_{a}\left|\prod\right| R_{a}$.

Then, $P$ can input $P_{i}: s, d \vdash P\left|\prod\right| ! \bar{a}\langle X\rangle . d \stackrel{\tau}{\rightarrow} s, d \vdash P^{\prime}\left|\prod\right| d$ and, weakly, we get $t_{a}, d \vdash Q_{a}\left|\prod\right| R_{a} \stackrel{\tau}{\Rightarrow} t_{b}, d \vdash Q_{b}\left|\prod\right| R_{b}$ and $s, d \vdash P^{\prime}\left|\prod\right| d \approx_{r+d}$ $t_{b}, d \vdash Q_{b}\left|\prod\right| R_{b}$ for some $R_{b}$ which has still to be one of ! $\bar{a}\left\langle{ }^{\prime} Q_{i}\right\rangle . d$ or $d$ because of clause 2 of Definition B.5 on barb $d$.

We can now spawn with clause 4 a process: $s, d \vdash P^{\prime}\left|\prod\right| d \mid \bar{d} \approx_{r+d} t_{b}, d \vdash$ $Q_{b}\left|\prod\right| R_{b} \mid \bar{d}$ and have a reaction: $s, d \vdash P\left|\prod\right| d\left|\bar{d} \stackrel{\tau}{\rightarrow} s, d \vdash P^{\prime}\right| \prod$ By clause 1 of Definition B.5, we have $t_{b}, d \vdash Q_{b}\left|\prod\right| R_{b}\left|\bar{d} \stackrel{\tau}{\Rightarrow} t^{\prime}, d \vdash Q^{\prime}\right| \prod$ and $s, d \vdash P^{\prime}\left|\prod \approx_{r+d} t^{\prime}, d \vdash Q^{\prime}\right| \prod$ since necessarily by clause 3 of Definition B.5, there must be no $d$ left in RHS otherwise LHS could not exhibit the same barb.

This means that $Q$ has weakly been able to input $Q_{i}$ on channel $a$ at some point, as: $Q \stackrel{\tau}{\Rightarrow} \stackrel{a\left({ }^{\circ} Q_{i}\right)}{\longrightarrow} \stackrel{\tau}{\Rightarrow} Q^{\prime}$. Therefore, we have $\left(\mathcal{E}_{x}, r_{x}, s_{x}, P^{\prime} \mid\right.$ $\left.a_{1}\left[P_{1}\right]|\ldots(\mid 0)| \ldots\left|a_{n}\left[P_{n}\right], t_{x}^{\prime}, Q^{\prime \prime}\right| a_{1}\left[Q_{1}\right]|\ldots(\mid 0)| \ldots \mid a_{n}\left[Q_{n}\right]\right) \in \mathcal{X}$.

6. $P_{j}$ passivates $a_{i}\left[P_{i}\right]$.

$s \vdash P\left|\prod \approx_{r} t \vdash Q\right| \prod$ so, for some fresh $d$ and $g$, by clause 4 of Definition B.5, for $e_{x}=d+g$ and $r^{\prime}=r+e_{x}$, we have $s, e_{x} \vdash$ $P\left|\prod\right| f_{i}(X) . ! \bar{a}\langle X\rangle . d \mid f_{j}(X) . g[$ runX $X] \approx_{r^{\prime}} t, e_{x} \vdash Q\left|\prod\right| f_{i}(X) . ! \bar{a}\langle X\rangle . d \mid$ $f_{j}(X) \cdot g[$ run $X]$.

Then, we can, as usual do a reaction between $! \overline{f_{i}}\langle\rangle$ and $f_{i}($ ) (which is followed by consuming $f_{i}$ ( ) too in RHS by clause 3 of Definition B.5, but does not consume $f_{j}()$ for the same reasons), then a reaction between $! \overline{f_{j}}\langle\rangle$ and $f_{j}$ which is followed, and we get $s, e_{x} \vdash P\left|\prod\right| f_{i}(X) \cdot ! \bar{a}\langle X\rangle . d \mid$ $f_{j}(X) . g\left[\right.$ runX $\stackrel{\tau}{\rightarrow}^{\rightarrow} \stackrel{\tau}{\rightarrow} s, e_{x} \vdash P\left|\prod\right| ! \bar{a}\left\langle{ }^{\prime} P_{i}\right\rangle . d \mid g\left[\right.$ run $\left.^{\natural} P_{j}\right], t, e_{x} \vdash Q\left|\prod\right| f_{i}(X) . ! \bar{a}\langle X\rangle . d \mid$

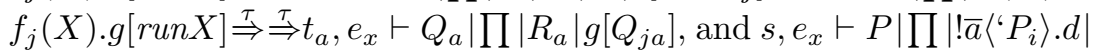
$g\left[\right.$ run $\left.^{\prime} P_{j}\right] \approx_{r^{\prime}} t_{a}, e_{x} \vdash Q_{a}\left|\prod\right| R_{a} \mid g\left[Q_{j a}\right]$ for some $R_{a}$ in $! \bar{a}\left\langle{ }^{\prime} Q_{i}\right\rangle . d, d$.

Then, we can do a run -transition, weakly followed as usual, to get $s, e_{x} \vdash$ $P\left|\prod\right| ! \bar{a}\left\langle{ }^{\prime} P_{i}\right\rangle . d \mid g\left[\right.$ run $\left.^{\prime} P_{j}\right] \stackrel{\text { run }}{\longrightarrow} \stackrel{\tau}{\rightarrow} s, e_{x} \vdash P\left|\prod\right| ! \bar{a}\left\langle{ }^{\prime} P_{i}\right\rangle . d \mid g\left[P_{j}\right], t_{a}, e_{x} \vdash$ $Q_{a}\left|\prod\right| R_{a}\left|g\left[Q_{j a}\right] \stackrel{\tau}{\Rightarrow} t_{b}, e_{x} \vdash Q_{b}\right| \prod\left|R_{b}\right| g\left[Q_{j b}\right]$, and $s, e_{x} \vdash P\left|\prod\right| ! \bar{a}\left\langle{ }^{\prime} P_{i}\right\rangle . d \mid$ $g\left[P_{j}\right] \approx_{r^{\prime}} t_{b}, e_{x} \vdash Q_{b}\left|\prod\right| R_{b} \mid g\left[Q_{j b}\right]$ for some $R_{b}$ in $! \bar{a}\left\langle{ }^{\prime} Q_{i}\right\rangle . d, d$.

Then, we can have $P_{j}$ input ' $P_{i}: s, e_{x} \vdash P\left|\prod\right| ! \bar{a}\left\langle{ }^{\prime} P_{i}\right\rangle . d \mid g\left[P_{j}\right] \stackrel{\tau}{\rightarrow} s, e_{x} \vdash$ $P\left|\prod\right| d \mid g\left[P_{j}^{\prime}\right]$, hence $t_{b}, e_{x} \vdash Q_{b}\left|\prod\right| R_{b} \mid g\left[Q_{j b} \stackrel{\sim}{\Rightarrow} t_{c}, e_{x} \vdash Q_{c}\left|\prod\right| R_{c} \mid g\left[Q_{j c}\right]\right.$ 
and $s, e_{x} \vdash P\left|\prod\right| d\left|g\left[P_{j}^{\prime}\right] \approx_{r^{\prime}} t_{c}, e_{x} \vdash Q_{c}\right| \prod\left|R_{c}\right| g\left[Q_{j c}\right]$ for some $R_{c}$ in $! \bar{a}\left\langle{ }^{\prime} Q_{i}\right\rangle . d, d$ because of clause 2 on barb $d$.

Then, we can remove the $d$ by spawning $\bar{d}$ and causing a reaction: $s, e_{x} \vdash$ $P\left|\prod\right| d\left|g\left[P_{j}^{\prime}\right]\right| \bar{d} \approx_{r^{\prime}} t_{c}, e_{x} \vdash Q_{c}\left|\prod\right| R_{c}\left|g\left[Q_{j c}\right]\right| \bar{d}$ and then $s, e_{x} \vdash P\left|\prod\right| d \mid$ $g\left[P_{j}^{\prime}\right]\left|\bar{d} \stackrel{\tau}{\rightarrow} s, e_{x} \vdash P\right| \prod \mid g\left[P_{j}^{\prime}\right]$, hence $t_{c}, e_{x} \vdash Q_{c}\left|\prod\right| R_{c}\left|g\left[Q_{j c}\right]\right| \bar{d} \stackrel{\tau}{\Rightarrow} t_{d}, e_{x} \vdash$ $Q_{d}\left|\prod\right| g\left[Q_{j d}\right]$, and $s, e_{x} \vdash P\left|\prod\right| g\left[P_{j}^{\prime}\right] \approx_{r^{\prime}} t_{d}, e_{x} \vdash Q_{d}\left|\prod\right| g\left[Q_{j d}\right]$ because RHS had to consume ! $\bar{a}\left\langle^{\prime} Q_{i}\right\rangle$ otherwise LHS could not exhibit barb $d$.

Now, we spawn another process with fresh $f_{n+1}$, and for $e_{x}^{\prime}=e_{x}+f_{n+1}$ and $r^{\prime \prime}=r+e_{x}^{\prime}$ we have $s, e_{x}^{\prime} \vdash P\left|\prod\right| g\left[P_{j}^{\prime}\right] \mid g(X) . ! \overline{f_{n+1}}\langle X\rangle \approx_{r^{\prime \prime}} t_{d}, e_{x}^{\prime} \vdash$ $Q_{d}\left|\prod\right| g\left[Q_{j d}\right] \mid g(X) ! \overline{f_{n+1}}\langle X\rangle$.

And we can do one last reaction which will be followed by the same reaction, and some internal reductions of $Q_{d}: s, e_{x}^{\prime} \vdash P\left|\prod\right| g\left[P_{j}^{\prime}\right] \mid g(X) . ! \overline{f_{n+1}}\langle X\rangle \stackrel{\tau}{\rightarrow}$ $s, e_{x}^{\prime} \vdash P\left|\prod\right| ! \overline{f_{n+1}}\left\langle{ }^{\prime} P_{j}\right\rangle, t_{d}, e_{x}^{\prime} \vdash Q_{d}\left|\prod\right| g\left[Q_{i d}\right] \mid g(X) ! \overline{f_{n+1}}\langle X\rangle \stackrel{\tau}{\Rightarrow}$ $t^{\prime}, e_{x}^{\prime} \vdash Q^{\prime}\left|\prod\right| ! \overline{f_{n+1}}\left\langle{ }^{\prime} Q_{i e}\right\rangle$ with $s, e_{x}^{\prime} \vdash P\left|\prod\right| ! \overline{f_{n+1}}\left\langle{ }^{\prime} P_{j}\right\rangle \approx_{r^{\prime \prime}} t^{\prime}, e_{x}^{\prime} \vdash$ $Q^{\prime}\left|\prod\right| ! \overline{f_{n+1}}\left\langle{ }^{\prime} Q_{i e}\right\rangle$ and $Q_{i e}$ being either $\operatorname{run}^{\prime} Q_{j}$ or $Q_{j}^{\prime}$.

There are two possible cases, either $Q$ did the input at some time, or $Q_{i}$ did it. If $Q$ has done the passivation, as in the above case, proper transitions exist and membership to $\mathcal{X}$ is guaranteed (via erasure of $r^{\prime} n^{'} Q_{j}$ 's run if necessary) If $Q_{j}$ has done the passivation, then necessarily its outer run has been consumed, and there is no need for any erasure. Again, this shows that the transition could be done by the member of $\mathcal{X}$, and that we still are in $\mathcal{X}$.

- Outputs

1. $P \stackrel{\bar{a}\langle M\rangle}{\longrightarrow} P^{\prime}$.

so, for fresh $g$ and $f_{n+1}$, using clause 4 of Definition B.5, for $e_{x}=g+f_{n+1}$ and $r^{\prime}=r+e_{x}$, we have $s, e_{x} \vdash P\left|\prod\right| a(X) \cdot g ! \overline{f_{n+1}}\langle X\rangle \approx_{r^{\prime}} t, e_{x} \vdash$ $Q\left|\prod\right| a(X) . g . ! f_{n+1}\langle X\rangle$.

We can have a reaction that consumes $a(X): s, e_{x} \vdash P\left|\prod\right| a(X) \cdot g . ! \overline{f_{n+1}}\langle X\rangle \stackrel{\tau}{\rightarrow}$ $s, e_{x} \vdash P^{\prime}\left|\prod\right| g . \overline{f_{n+1}}\langle M\rangle$, followed weakly: $t, e_{x} \vdash Q\left|\prod\right| a(X) . g . ! \overline{f_{n+1}}\langle X\rangle \stackrel{\tau}{\Rightarrow}$ $t_{a}, e_{x} \vdash Q_{a}\left|\prod\right| R_{a}$ such that $s, e_{x} \vdash P^{\prime}\left|\prod\right| g . ! f_{n+1}\langle M\rangle \approx_{r^{\prime}} t_{a}, e_{x} \vdash$ $Q_{a}\left|\prod\right| R_{a}$ with $R_{a}$ one of $a(X) \cdot g . ! f_{n+1}\langle X\rangle$ or $g . ! f_{n+1}\langle X\rangle$ since clause 2 of Definition B.5 enforces that $R_{a}$ has barb $g$.

Then, we can spawn $\bar{g}: s, e_{x} \vdash P^{\prime}\left|\prod\right| g ! \overline{f_{n+1}}\langle M\rangle\left|\bar{g} \approx_{r^{\prime}} t_{a}, e_{x} \vdash Q_{a}\right| \prod\left|R_{a}\right|$ $\bar{g}$ and have a reaction to remove $g$ and $\bar{g}: s, e_{x} \vdash P^{\prime}\left|\prod\right| g . ! \overline{f_{n+1}}\langle M\rangle \mid \bar{g} \stackrel{\tau}{\rightarrow}$ $s, e_{x} \vdash P^{\prime}\left|\prod\right| ! \overline{f_{n+1}}\langle M\rangle$ which is followed by $t_{a}, e_{x} \vdash Q_{a}\left|\prod\right| R_{a} \mid \bar{g} \stackrel{\tau}{\Rightarrow} t^{\prime}, e_{x} \vdash$ $Q^{\prime}\left|\prod\right| R^{\prime}$ such that $s, e_{x} \vdash P^{\prime}\left|\prod\right| ! \overline{f_{n+1}}\langle M\rangle \approx_{r^{\prime}} t^{\prime}, e_{x} \vdash Q^{\prime}\left|\prod\right| R^{\prime}$ with necessarilly $R^{\prime}=! \overline{f_{n+1}}\langle N\rangle$ since LHS has no barb $g$ left.

This means that $Q$ has weakly been able to output $N$ on channel $a$ at some point, that is that $t_{x} \vdash Q\left|a_{1}\left[Q_{1}\right]\right| \ldots\left|a_{n}\left[Q_{n}\right] \stackrel{\bar{a}\langle N\rangle}{\Longrightarrow} t_{x}^{\prime} \vdash Q^{\prime}\right|$ $a_{1}\left[Q_{1}\right]|\ldots| a_{n}\left[Q_{n}\right]$ and, as it happens, $\left((M, N) \oplus \mathcal{E}_{x}, r_{x}, s_{x}, P^{\prime}\left|a_{1}\left[P_{1}\right]\right|\right.$ $\left.\ldots\left|a_{n}\left[P_{n}\right], t_{x}^{\prime}, Q^{\prime}\right| a_{1}\left[Q_{1}\right]|\ldots| a_{n}\left[Q_{n}\right]\right) \in \mathcal{X}$ since $(M, N)$ is now in $\mathcal{E}$.

2. Passivation of $a\left[P_{i}\right]$.

Immediate.

3. $P_{i} \stackrel{\bar{a}\langle M\rangle}{\longrightarrow} P_{i}^{\prime}$. 
For fresh $g$, using clause 4 of Definition B.5, we have $s, g \vdash P\left|\prod\right| f_{i}(X) \cdot g\left[\right.$ runX $X \approx_{r+g}$ $t, g \vdash Q\left|\prod\right| f_{i}(X) . g[\operatorname{run} X]$.

We can do a reaction to consume $f_{i}()$ and get $s, g \vdash P\left|\prod\right| f_{i}(X) . g[$ runX $X \stackrel{\tau}{\rightarrow}$ $s, g \vdash P\left|\prod\right| g\left[\right.$ run $\left.^{6} P_{i}\right]$, hence $t, g \vdash Q\left|\prod\right| f_{i}(X) . g[$ runX $] \stackrel{\tau}{\Rightarrow} t_{a}, g \vdash$ $Q_{a}\left|\prod\right| g\left[Q_{i a}\right]$ and $s, g \vdash P\left|\prod\right| g\left[\right.$ run $\left.^{6} P_{i}\right] \approx_{r+g} t_{a}, g \vdash Q_{a}\left|\prod\right| g\left[Q_{i a}\right]$.

Then, we can remove the run: $s, g \vdash P\left|\prod\right| g\left[\right.$ run $\left.^{6} P_{i}\right] \stackrel{\text { run }}{\longrightarrow} \stackrel{\tau}{\rightarrow} s, g \vdash P \mid$ $\prod \mid g\left[P_{i}\right]$, hence $t_{a}, g \vdash Q_{a}\left|\prod\right| g\left[Q_{i a}\right] \stackrel{\tau}{\Rightarrow} t_{b}, g \vdash Q_{b}\left|\prod\right| g\left[Q_{i b}\right]$ and $s, g \vdash$ $P\left|\prod\right| g\left[P_{i}\right] \approx_{r+g} \stackrel{\tau}{\Rightarrow} t_{b}, g \vdash Q_{b}\left|\prod\right| g\left[Q_{i b}\right]$.

We can now spawn a receiver on $a$ with fresh names $f_{n+1}$ and $f_{n+2}$. For $e_{x}=g+f_{n+1}+f_{n+2}$ and $r^{\prime}=r+e_{x}$, we have: $s, e_{x} \vdash P$ $\prod\left|g\left[P_{i}\right]\right| a(X) \cdot g(Y)\left(! \overline{f_{n+1}}\langle X\rangle \mid ! \overline{f_{n+2}}\langle Y\rangle\right) \approx_{r^{\prime}} t_{b}, e_{x} \vdash Q_{b}\left|\prod\right| g\left[Q_{i b}\right] \mid$ $a(X) \cdot g(Y)\left(! f_{n+1}\langle X\rangle \mid ! \overline{f_{n+2}}\langle Y\rangle\right)$ for $r^{\prime}=r+e_{x}$, and have LHS react on $a(X): s, e_{x} \vdash P\left|\prod\right| g\left[P_{i}\right] \mid a(X) \cdot g(Y)\left(\overline{f_{n+1}}\langle X\rangle \mid f_{n+2}\langle Y\rangle\right) \stackrel{\tau}{\rightarrow} s, e_{x} \vdash$ $P\left|\prod\right| g\left[P_{i}^{\prime}\right] \mid g(Y)\left(! f_{n+1}\langle M\rangle \mid ! \overline{f_{n+2}}\langle Y\rangle\right)$ hence $t_{b}, e_{x} \vdash Q_{b}\left|\prod\right| g\left[Q_{i b}\right] \mid$ $a(X) . g(Y)\left(\overline{f_{n+1}}\langle X\rangle \mid ! \overline{f_{n+2}}\langle Y\rangle\right) \stackrel{\sim}{\Rightarrow} t_{c}, e_{x} \vdash Q_{c}\left|\prod\right| g\left[Q_{i b}\right] \mid R_{c}$ and $s, e_{x} \vdash$ $P\left|\prod\right| g\left[P_{i}^{\prime}\right]\left|g(Y)\left(! \overline{f_{n+1}}\langle M\rangle \mid ! \overline{f_{n+2}}\langle Y\rangle\right) \approx_{r^{\prime}} t_{c}, e_{x} \vdash Q_{c}\right| \prod\left|g\left[Q_{i b}\right]\right| R_{c}$ with $R_{c}$ in $a(X) \cdot g(Y) \cdot\left(! f_{n+1}\langle X\rangle \mid ! f_{n+2}\langle Y\rangle\right)$ or $g(Y) \cdot\left(! \overline{f_{n+1}}\langle N\rangle \mid ! \bar{f}_{n+2}\langle Y\rangle\right)$ because LHS still has barb $g$.

Then, LHS can react on $g: s, e_{x} \vdash P\left|\prod\right| g\left[P_{i}^{\prime}\right] \mid g(Y)\left(! \overline{f_{n+1}}\langle M\rangle \mid \overline{f_{n+2}}\langle Y\rangle\right) \stackrel{\tau}{\rightarrow}$ $s, e_{x} \vdash P\left|\prod\right| ! \overline{f_{n+1}}\langle M\rangle \mid ! \overline{f_{n+2}}\left\langle{ }^{\prime} P_{i}^{\prime}\right\rangle$, hence $t_{c}, e_{x} \vdash Q_{c}\left|\prod\right| g\left[Q_{i b}\right] \mid R_{c} \stackrel{\tau}{\Rightarrow}$ $t^{\prime}, e_{x} \vdash Q^{\prime}\left|\prod\right| ! \bar{f}_{n+1}\langle N\rangle \mid ! f_{n+2}\left\langle{ }^{\prime} Q_{i c}\right\rangle$ and $s, e_{x} \vdash P\left|\prod\right| ! f_{n+1}\langle M\rangle \mid$ $\overline{! f_{n+2}}\left\langle{ }^{\prime} P_{i}^{\prime}\right\rangle \approx_{r^{\prime}} t^{\prime}, e_{x} \vdash Q^{\prime}\left|\prod\right| ! f_{n+1}\langle N\rangle \mid ! \overline{f_{n+2}}\left\langle{ }^{\prime} Q_{i c}\right\rangle$ since LHS has no more barb on $g$, which enforced the reaction with $a(X)$ in $R_{c}$ or its 'parent' at some point.

Again, $Q_{i c}$ is either run' $Q_{i}$ or some $Q_{i}^{\prime}$. If it is $\operatorname{run}^{\prime} Q_{i}$, then we can use run-erasure of $\approx$ and we are done since it was not used in the transitions. If not, then its outer has been consumed, and we're done.

Again, this shows that the transition could be followed by RHS of the member of $\mathcal{X}$, and that we still are in $\mathcal{X}$.

- Inputs

1. $P \stackrel{a(M)}{\longrightarrow} P^{\prime}$.

For some fresh $d$, and some $O$ such that $f n(O) \subseteq r_{x}, O\left\{{ }^{\prime} P_{a} / X_{a}, \ldots,{ }^{\prime} P_{b} / X_{b}\right\}=$ $M$, we have $s, d \vdash P\left|\prod\right| f_{a}\left(X_{a}\right) \ldots \ldots f_{b}\left(X_{b}\right) \cdot \bar{a}\langle O\rangle . d \approx_{r+d} t, d \vdash Q \mid$ $\prod \mid f_{a}\left(X_{a}\right) \ldots . f_{b}\left(X_{b}\right) \cdot \bar{a}\langle O\rangle . d$.

We can do all the reactions on $f_{a}(), \ldots, f_{b}()$, and they'll be followed by clauses 2 and 3 on barbs, hence we have $s, d \vdash P\left|\prod\right| f_{a}\left(X_{a}\right) \ldots f_{b}\left(X_{b}\right) \cdot \bar{a}\langle O\rangle . d \stackrel{\tau}{\rightarrow}$ $\ldots \stackrel{\tau}{\rightarrow} s, d \vdash P\left|\prod\right| \bar{a}\langle M\rangle . d t, d \vdash Q\left|\prod\right| f_{a}\left(X_{a}\right) \ldots . . f_{b}\left(X_{b}\right) \cdot \bar{a}\langle O\rangle . d \stackrel{\tau}{\Rightarrow} \ldots \stackrel{\tau}{\Rightarrow}$ $t_{a}, d \vdash Q_{a}\left|\prod\right| R_{a}, s, d \vdash P\left|\prod\right| \bar{a}\langle M\rangle . d \approx_{r+d} t_{a}, d \vdash Q_{a}\left|\prod\right| R_{a}$ with $R_{a}$ being $\bar{a}\langle N\rangle . d$ or $d$ by clause 2 on barb $d$ and 3 on $f_{b}$.

Then, LHS can react on $\bar{a}\langle M\rangle: s, d \vdash P\left|\prod\right| \bar{a}\langle M\rangle . d \stackrel{\tau}{\rightarrow} s, d \vdash P^{\prime}\left|\prod\right| d$ hence $t_{a}, d \vdash Q_{a}\left|\prod\right| R_{a} \stackrel{\tau}{\Rightarrow} t_{b}, d \vdash Q_{b}\left|\prod\right| R_{b}, s, d \vdash P^{\prime}\left|\prod\right| d \approx_{r+d} t_{b}, d \vdash Q_{b}\left|\prod\right| R_{b}$ with $R_{b}$ being $\bar{a}\langle N\rangle . d$ or $d$ by clause 2 on barb $d$.

By clause 4 of Definition B.5, we can spawn $\bar{d}: s, d \vdash P^{\prime}\left|\prod\right| d \mid \bar{d} \approx_{r+d}$ $t_{b}, d \vdash Q_{b}\left|\prod\right| R_{b} \mid \bar{d}$ and have a reaction: $s, d \vdash P^{\prime}\left|\prod\right| d\left|\bar{d} \stackrel{\tau}{\rightarrow} s, d \vdash P^{\prime}\right| \prod$ 
hence $t_{b}, d \vdash Q_{b}\left|\prod\right| R_{b}\left|\bar{d} \stackrel{\tau}{\Rightarrow} t^{\prime}, d \vdash Q^{\prime}\right| \prod$ with $s, d \vdash P^{\prime} \mid \prod \approx_{r+d} t^{\prime}, d \vdash$ $Q^{\prime} \mid \prod$ by the clause 3 of Definition B.5 on barb $d$.

Again, this shows that the transition could be done by the member of $\mathcal{X}$, and that we still are in $\mathcal{X}$.

2. $P_{i} \stackrel{a(M)}{\longrightarrow} P_{i}^{\prime}$.

For some fresh $d$, and some $O$ such that $f n(O) \subseteq r_{x}, O\left\{{ }^{\prime} P_{a} / X_{a}, \ldots,{ }^{'} P_{b} / X_{b}\right\}=$ $M$, we have $s, d \vdash P\left|\prod\right| f_{a}\left(X_{a}\right) \ldots \ldots f_{b}\left(X_{b}\right) \cdot \bar{a}\langle O\rangle \cdot d \approx_{r+d} t, d \vdash Q \mid$ $\prod \mid f_{a}\left(X_{a}\right) \ldots f_{b}\left(X_{b}\right) \cdot \bar{a}\langle O\rangle . d$. We can do all the reactions on $f_{a}(), \ldots, f_{b}()$, and they'll be followed by clauses 2 and 3 on barbs, hence we have $s, d \vdash P\left|\prod\right| f_{a}\left(X_{a}\right) \ldots . f_{b}\left(X_{b}\right) . \bar{a}\langle O\rangle . d \stackrel{\tau}{\rightarrow} \ldots \stackrel{\tau}{\rightarrow} s, d \vdash P\left|\prod\right| \bar{a}\langle M\rangle . d$ and $t, d \vdash Q\left|\prod\right| f_{a}\left(X_{a}\right) \ldots f_{b}\left(X_{b}\right) . \bar{a}\langle O\rangle . d \stackrel{\tau}{\Rightarrow} \ldots \stackrel{\tau}{\Rightarrow} t_{a}, d \vdash Q_{a}\left|\prod\right| R_{a}$ and $s, d \vdash P\left|\prod\right| \bar{a}\langle M\rangle . d \approx_{r+d} t_{a}, d \vdash Q_{a}\left|\prod\right| R_{a}$ with $R_{a}$ being $\bar{a}\langle N\rangle . d$ or $d$ by clause 2 on barb $d$.

We can now spawn with clause 4 of Definition B.5 a process with free name g, and, for $e_{x}=d+g$ and $r^{\prime}=r+e_{x}$, erase the run's from $s, e_{x} \vdash$ $P\left|\prod\right| \bar{a}\langle M\rangle . d \mid f_{i}(X) . g[$ runX $X] \approx_{r^{\prime}} t_{a}, e_{x} \vdash Q_{a}\left|\prod\right| R_{a} \mid f_{i}(X) . g[$ runX $X]$ to get $s, e_{x} \vdash P\left|\prod\right| \bar{a}\langle M\rangle . d\left|g\left[P_{i}\right] \approx_{r^{\prime}} t_{a a}, e_{x} \vdash Q_{a a}\right| \prod\left|R_{a a}\right| g\left[Q_{i a}\right]$ by clause 3 of Definition B.5 on barb $f_{i}$ and 2 on $g$.

Then, LHS can react on $\bar{a}\langle M\rangle: s, e_{x} \vdash P\left|\prod\right| \bar{a}\langle M\rangle . d\left|g\left[P_{i}\right] \stackrel{\tau}{\rightarrow} s, e_{x} \vdash P\right|$ $\prod|d| g\left[P_{i}^{\prime}\right]$ hence $t_{a a}, e_{x} \vdash Q_{a} a\left|\prod\right| R_{a} a\left|g\left[Q_{i a a}\right] \stackrel{\tau}{\Rightarrow} t_{b}, e_{x} \vdash Q_{b}\right| \prod\left|R_{b}\right| g\left[Q_{i b}\right]$, and $s, e_{x} \vdash P\left|\prod\right| d\left|g\left[P_{i}^{\prime}\right] \approx_{r^{\prime}} t_{b}, e_{x} \vdash Q_{b}\right| \prod\left|R_{b}\right| g\left[Q_{i b}\right]$ with $R_{b}$ being $\bar{a}\langle N\rangle . d$ or $d$ by (now) clause 2 (too) on barb $d$.

By clause 4 of Definition B.5, we can spawn $\bar{d}: s, e_{x} \vdash P\left|\prod\right| d\left|g\left[P_{i}^{\prime}\right]\right| \bar{d} \approx_{r^{\prime}}$ $t_{b}, e_{x} \vdash Q_{b}\left|\prod\right| R_{b}\left|g\left[Q_{i b}\right]\right| \bar{d}$ and have a reaction: $s, e_{x} \vdash P\left|\prod\right| d|\bar{d}| g\left[P_{i}^{\prime}\right] \stackrel{\tau}{\rightarrow}$ $s, e_{x} \vdash P\left|\prod\right| g\left[P_{i}^{\prime}\right]$ hence $t_{b}, e_{x} \vdash Q_{b}\left|\prod\right| R_{b}\left|g\left[Q_{i b}\right]\right| \bar{d} \stackrel{\tau}{\Rightarrow} t_{c}, e_{x} \vdash Q_{c}\left|\prod\right| g\left[Q_{i c}\right]$ with $s, e_{x} \vdash P\left|\prod\right| g\left[P_{i}^{\prime}\right] \approx_{r^{\prime}} t_{c}, e_{x} \vdash Q_{c}\left|\prod\right| g\left[Q_{i c}\right]$ by the clause 3 of Definition B.5 on barb $d$, which enforced the reaction on channel $a$ in $R_{b}$ (or its parent) in the RHS.

Finally, to put $P_{i}^{\prime}$ and $Q_{i c}$ in the environment, we spawn a process with free name $f_{n+1}$, and for $e_{x}^{\prime}=e_{x}+f_{n+1}$ and $r^{\prime \prime}=r+e_{x}^{\prime}$ we have: $s, e_{x}^{\prime} \vdash$ $P\left|\prod\right| g\left[P_{i}^{\prime}\right]\left|g(X) . ! \overline{f_{n+1}}\langle X\rangle \approx_{r^{\prime \prime}} t_{c}, e_{x}^{\prime} \vdash Q_{c}\right| \prod\left|g\left[Q_{i c}\right]\right| g(X) . ! \overline{f_{n+1}}\langle X\rangle$ and derive, as expected, $s, e_{x}^{\prime} \vdash P\left|\prod_{n+1} \approx_{r^{\prime \prime}} t_{c}, e_{x}^{\prime} \vdash Q_{c}\right| \prod_{n+1}$ with the right transitions, using the run-erasure if necessary.

Again, this shows that the transition could be done by the member of $\mathcal{X}$, and that we still are in $\mathcal{X}$.

- Spawn clause.

Immediate by definition of $\mathcal{X}$.

- Name creation.

We can add any name not in $s, t$ to $r$ (hence not in $s_{x}, t_{x}$, to $r_{x}$ ), so that they do not clash with other names, by choosing another $r$.

- Converses of 1,2 and 3.

Similarly.

Corollary B.51. [Completeness of environmental bisimulation] If $f \vdash P \approx_{r} f \vdash Q$ with $r \subseteq f=f n(P, Q)$, then $f \vdash P \sim_{\emptyset ; r} f \vdash Q$. 
Proof. From Lemma B.50.

Corollary B.52. [Completeness of environmental bisimulation w.r.t. reductionclosed barbed congruence]

If $f, a \vdash P \dot{\approx}_{r a} f, a \vdash Q$ with $r \subseteq f=f n(P, Q)$, then $f, a \vdash \bar{a}\langle P\rangle \sim_{\emptyset ; r a} f, a \vdash$ $\bar{a}\langle Q\rangle$.

Proof. By $f, a \vdash P \dot{\approx}_{r a} f, a \vdash Q$, we have $f, a \vdash \bar{a}\langle P\rangle \dot{\approx}_{r a} f, a \vdash \bar{a}\langle Q\rangle$, hence $f, a \vdash \bar{a}\langle P\rangle \approx_{r a} f, a \vdash \bar{a}\langle Q\rangle$, hence $f, a \vdash \bar{a}\langle P\rangle \sim_{\emptyset ; r a} f, a \vdash \bar{a}\langle Q\rangle$ by Corollary B.51.

Definition B.53. We write $P \bumpeq_{r} Q$ if $(f \vdash 0) \sim_{\left\{\left({ }^{(} P,{ }^{\prime} Q\right)\right\} ; r}(f \vdash 0)$ with $r \subseteq f=$ $f n(P, Q)$.

Corollary B.54. [Characterisation of reduction-closed barbed congruence] $P \bumpeq_{r} Q$ if and only if $f \vdash P \dot{\sim}_{r} f \vdash Q$ with $r \subseteq f=f n(P, Q)$.

Proof. $(\Rightarrow)$ : from B.47.

$(\Leftarrow)$ : from B.52, and then by output to $a$. 